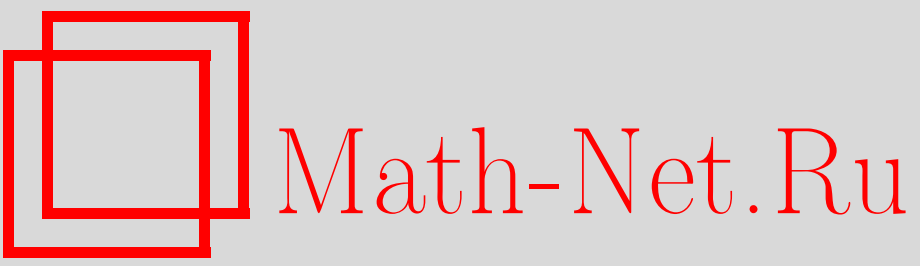

А. М. Семихатов, Представления бесконечномерных алгебр и конформная теория поля: от $N=2$ до $\widehat{s l}(2 \mid 1), T M \Phi, 1997$, том 112, номер 2, 195-240

DOI: https://doi.org/10.4213/tmf1039

Использование Общероссийского математического портала Math-Net.Ru подразумевает, что вы прочитали и согласны с пользовательским соглашением

http://www.mathnet.ru/rus/agreement

Параметры загрузки:

IP : 3.80 .253 .173

26 апреля 2023 г., 13:01:00 


\section{ПРЕДСТАВЛЕНИЯ БЕСКОНЕЧНОМЕРНЫХ АЛГЕБР И КОНФОРМНАЯ ТЕОРИЯ ПОЛЯ: ОТ $N=2$ ДО $\widehat{s l}(2 \mid 1)^{1)}$}

Дается обзор ряда теоретико-представленческих конструкций, реализуемых в бозонных и $N=2$ струнах и связывающих аффинную алгебру Ли $\widehat{s l}(2)$, аффинную супералгебру Ли $\widehat{s l}(2 \mid 1)$ и суперконформную $N=2$ алгебру.

\section{1. ВВЕДЕНИЕ}

Настояшая работа посвящена представлениям трех бесконечномерных алгебр, популярных в конформной теории поля и теории струн: $N=2$ суперконформной и аффинных алгебр $\widehat{s l}(2)$ и $\widehat{s l}(2 \mid 1)$. Коль скоро речь будет идти о конформных теориях, во многих конструкциях будет явно или неявно присутствовать алгебра Вирасоро.

Алгебра Вирасоро является наиболее полно изученной из перечисленных алгебр. Все ее неприводимые представления являются фактор-модулями модулей Верма, а подмодули последних характеризуются сингулярными векторами (чем и определяется постоянный интерес к структуре и явным конструкциям для сингулярных векторов не только над алгеброй Вирасоро, но и над другими бесконечномерными алгебрами [1-11]), которые классифицированы и для которых имеются процедуры явного построения; известны также БГГ-резольвента для алгебры Вирасоро и диаграммы вложений модулей Верма. Многие из этих математических “данных" тем или иным образом используются в минимальных моделях [12]. Напомним еще, что алгебра Вирасоро реализована на мировом листе бозонной (и всякой другой) струны, причем в каждом из секторов - материи, духов и (для некритических струн) лиувиллевского скаляра. Алгебра Вирасоро, однако, не является алгеброй Каца-Муди (KM).

Тесно связанной с алгеброй Вирасоро оказывается аффинная алгебра Ли $\widehat{s l}(2)$. Все ее неприводимые представления являются также факторами модулей Верма. Положения сингулярных векторов в модулях Верма следуют из анализа детерминанта Каца-Каждана [1], а, кроме того, имеются замечательные формулы Маликова-Фейгина-Фукса $(\mathrm{M} \Phi \Phi)[2]$, дающие все сингулярные векторы (во всяком случае, при $k \neq-2$ ) в виде мономов, содержащих, однако, комплексные степени генераторов; при этом формулы МФ $\Phi$ приобретают смысл, как только указываются алгебраические правила оперирования с

\footnotetext{
1) Статья написана по заказу Редколлегии.
}

* Отделение теоретической физики им. И.Е. Тамма, Физический институт им. П. Н. Лебедева РАН, Москва, Россия 
комплексными степенями. Связь с алгеброй Вирасоро выражается посредством гамильтоновой редукции и ее "обрашения" путем умножения алгебры Вирасоро и ее модулей Верма на теории свободных полей $[13,14]$. Модели Весса-Зумино-Новикова-Виттена (ВЗНВ), основанные на алгебре $\widehat{s l}(2)$, являются одним из популярных примеров конформных теорий поля. Правила слияния в них изучались сначала по аналогии с таковыми для алгебры Вирасоро, но со временем было обнаружено, что необходимо введение более общих модулей, чем стандартно рассматриваемые (т.е. те, которые получаются факторизацией обычных модулей Верма [15-17]). Здесь мы встречаемся с примером того, что определение категории рассматриваемых представлений имеет весьма непосредственные физические следствия.

В отличие от $\widehat{s l}(2)$ суперконформная $N=2$ алгебра в двух измерениях не только не является алгеброй КМ, но и оказывается существенно более сложной, чем алгебра Вирасоро. Устройство $N=2$ модулей было известно в ряде случаев, тогда как ни БГГ-резольвента, ни правила слияния полностью не известны. Позиции сингулярных векторов были получены из анализа соответствуюшего детерминанта Каца [10], но явное построение сингулярных векторов оказывается связанным с деталями структуры $N=2$ модулей, которые не повторяют структуру модулей над алгеброй Вирасоро или обычно рассматриваемых $\widehat{s l}(2)$-модулей. При этом, однако, имеется несколько указаний на то, что $N=2$ алгебра тесно связана с $\widehat{s l}(2)$, например, из обеих алгебр можно получить парафермионы, а из $\widehat{s l}(2)$-токов и свободных фермионов можно построить $N=2$ генераторы (отображение Кадзамы-Судзуки $(\mathrm{KC})$ ). Тем не менее, теоретико-представленческое понимание того, каким образом связаны модули над этими алгебрами, имеющими разный ранг, до недавнего времени отсутствовало.

Интерес к $N=2$ алгебре определяется, в частности, тем, что она реализована на мировом листе некритической бозонной (а тем самым и всякой другой) струны [18-20]. Кроме того, $N=2$ струна [21-24] вызывает в последнее время интерес из-за своей возможной роли в М-теории $[25,26]$ (об $N=2$ суперконформной алгебре см. также работы $[10,21,27-29])$.

Аффинная супералгебра $\widehat{s l}(2 \mid 1)$ - довольно привлекательный объект в теории представлений “низкоразмерных" (супер)алгебр КМ и в конструкциях, связанных с теорией струн. Она содержит равное количество (по 4) бозонных и фермионных токов; центральный заряд сугаваровского тензора энергии-импульса оказывается равным нулю. Алгебра $\widehat{s l}(2 \mid 1)$, конечно, содержит $\widehat{s l}(2)$ в качестве подалгебры. С другой стороны, ее гамильтонова редукция дает $N=2$ суперконформную алгебру [20,30,31], так что, в частности, алгебра $\widehat{s l}(2 \mid 1)$ "накрывает" некритическую бозонную струну [20]. Однако поведение представлений при подобных операторных конструкциях в конформной теории поля исследовалось недостаточно, в то время как знания такого рода необходимы для анализа физических состояний (БРСТ-когомологий) и соответствий между ними в различных теориях.

В настоящей статье устанавливаются результаты, демонстрируюшие достаточно глубокое родство между представлениями трех обсуждаемых бесконечномерных алгебр. Мы увидим, что отображение КС устанавливает соответствие между $\widehat{s l}(2)$ и $N=$ 2 представлениями, близкое к эквивалентности (а именно, оказываюшееся эквивалентностью после факторизации по спектральным потокам); с другой стороны, будут продемонстрированы родственная структура $N=2$ и $\widehat{s l}(2 \mid 1)$-модулей и взаимоотношение между соответствуюшими сингулярными векторами (которое окажется с контролиру- 
емым исключением отображением $2: 1$ ).

Изложение основано на работах [14, 32-36].

Мы начинаем с напоминания о стандартных модулях Верма над $\widehat{s l}(2)$, вводим в рассмотрение спектральный поток на $\widehat{s l}(2)$-алгебре и его действие на представлениях и определяем "скрученные" (твистованные) $\widehat{s l}(2)$-модули Верма. Вслед за этим мы определяем новый класс "расслабленных" (relaxed) модулей Верма над афффинной алгеброй $\widehat{s l}(2)$. Мы приведем структурную теорию этих модулей, а именно опишем встречающиеся в них сингулярные векторы, а также изучим свойства тех модулей, в которых сосуществуют разные типы сингулярных векторов. Как и в стандартной М $\Phi$-конструкции, сингулярные векторы даются формулами с комплексными степенями, но для расслабленных модулей возможное поведение построенных таким образом сингулярных векторов усложняется.

Для $N=2$ суперконформной алгебры мы построим “фермионный” аналог алгебраической теории комплексных степеней. Фермионные генераторы $N=2$ алгебры могут быть продолжены до "несобственных" операторов, в терминах которых сингулярные векторы записываются просто как мономы. Имеется набор алгебраических правил, позволяюших переписать мономиальные выражения явным образом в виде элементов из модуля Верма. Впрочем, само понятие $N=2$ модулей Верма требует уточнения. Необходимо различать два их вида: “топологические" и "массивные" (а также их "скручивания" под действием $N=2$ спектрального потока). Массивные $N=2$ модули Верма имеют бесконечное число векторов старшего веса.

Топологические модули Верма над $N=2$ алгеброй обладают следующим замечательным свойством: с точностью до соответствующих спектральных потоков категория скрученных $\widehat{s l}(2)$-модулей Верма эквивалентна категории скрученных топологических $N=2$ модулей. Категория же массивных $N=2$ модулей Верма оказывается (опять же с точностью до соответствующих спектральных потоков) эквивалентной категории расслабленных модулей Верма над $\widehat{s l}(2)$. Сингулярные векторы в $N=2$ и $\widehat{s l}(2)$-модулях взаимно однозначно соответствуют друг другу и до факторизации по спектральному потоку. В частности, конструкция $N=2$ сингулярных векторов в виде мономов по "продолженным" фермионным операторам оказывается изоморфной конструкции в терминах комплексных степеней для $\widehat{s l}(2)$-сингулярных векторов.

С необходимыми модификациями метод “алгебраического продолжения" применим и к построению сингулярных векторов аффинной супералгебры $\widehat{s l}(2 \mid 1)$. Алгебра $\widehat{s l}(2 \mid 1)$ в известном смысле комбинирует свойства $\widehat{s l}(2)$ и $N=2$ алгебр, и это будет хорошо видно в приложении к сингулярным векторам: чисто фермионная, как в $N=2$ случае, конструкция для $\widehat{s l}(2 \mid 1)$-сингулярных векторов немедленно переписывается в виде, уже не содержашем продолженных фермионных операторов, но зато содержащем комплексные степени бозонных генераторов.

Таким образом, $\widehat{s l}(2 \mid 1)$-алгебра оказывается родственной $N=2$ и $\widehat{s l}(2)$-алгебрам и на “структурном" уровне. Это подчеркивается еще одной конструкцией, уникальной для $\widehat{s l}(2 \mid 1)$ : эта алгебра реализуется непосредственно на мировом листе $N=2$ струны, так что одевание произвольных полей $N=2$ материи в $N=2$ струну дает обращение [13] гамильтоновой редукции $\widehat{s l}(2 \mid 1) \rightarrow N=2$. Мы приведем явные формулы для подобной реализации алгебры $\widehat{s l}(2 \mid 1)$ в терминах $N=2$ материи и свободных полей. Она оказывается согласованной со структурой подмодулей модулей Верма, т.е. сингулярных векторов. Полученные с помошью метода комплексных степеней сингулярные 
векторы супералгебры $\widehat{s l}(2 \mid 1)$ могут быть вычислены в указанной реализации и оказываются в отношении $2: 1$ к сингулярным векторам $N=2$ суперконформной алгебры сингулярные векторы в двух различных $\widehat{s l}(2 \mid 1)$-модулях редуцируются к одному и тому же $N=2$ сингулярному вектору (за исключением одной серии $\widehat{s l}(2 \mid 1)$-сингулярных векторов, занумерованных натуральными числами, которые не соответствуют никакому $N=2$ сингулярному вектору).

Общим для всех трех алгебр является наличие отображения спектрального потока (важность которого для $N=2$ алгебры, а также для (критических) $N=2$ струн отмечалась в $[24,29,37,38])$. Сушествование спектрального потока в известном смысле лежит в основе конструкции скрученных модулей Верма.

Отдельно следует сказать об экстремальных векторах и диаграммах таких векторов, так называемых әкстремальных диаграммах, с помошью которых мы исследуем модули над всеми тремя алгебрами. Анализ экстремальных векторов немедленно дает определенные серии сингулярных векторов, по историческим причинам называемых “заряженными”. Идея рассматривать экстремальные векторы была выдвинута в [39], где было отмечено, что в их терминах естественно переформулируется целый ряд теоретико-представленческих вопросов.

Эквивалентность категорий $\widehat{s l}(2)$ и $N=2$ модулей в определенном смысле происходит из изоморфизма соответствующих экстремальных диаграмм. Более того, тот факт, что такая эквивалентность в действительности имеет место лишш с точностью до спектрального потока, связан с тем обстоятельством, что спектральный поток на $N=2$ модулях Верма, действуя “вдоль” экстремальных диаграмм, перечисляет бесконечный набор спектрально повернутых модулей Верма.

Сингулярные векторы, с которыми мы оперируем, удовлетворяют “скрученным" (спектрально повернутым) условиям старшего веса, а не в точности тем же условиям, которые наложены на вектор старшего веса, порождаюший данный модуль Верма. Подобное расширение определения приводит к сингулярным векторам, которые порождают подмодули, заведомо не меньшие, чем те, что порождаются “стандартными" сингулярными векторами. В ряде случаев (а для $N=2$ алгебры всегда) сингулярные векторы в нашем смысле порождают максимальные подмодули даже тогда, когда стандартные сингулярные векторы порождают не максимальные подмодули и для описания структуры подмодулей требуется введение субсингулярных векторов. Как рассматриваемые нами, так и "стандартные" сингулярные векторы лежат на экстремальной диаграмме подмодуля. В случае общего положения все векторы на одной и той же экстремальной диаграмме порождают один и тот же подмодуль. Однако в ряде специальных случаев оказывается, что часть из состояний на экстремальной диаграмме порождает меньший подмодуль, чем другие состояния на той же диаграмме; среди первых могут оказаться "стандартные" сингулярные векторы, но никогда - рассматриваемые нами сингулярные векторы, удовлетворяюшие "скрученным" условиям старшего веса. Заметим еше, что понимаемые расширительно $N=2$ сингулярные векторы в точности соответствуют "стандартным" $\widehat{s l}(2)$-сингулярным векторам, которые порождают максимальные подмодули, так что вопроса о субсингулярных векторах для алгебры $\widehat{s l}(2)$ не возникает; подобным же образом рассматриваемые нами сингулярные векторы избавляют нас, по крайней мере для $N=2$ алгебры, от понятия субсингулярных векторов. 
2. МОДУЛИ ВЕРМА НАД АЛГЕБРАМИ $\widehat{s l}(2), N=2$ И $\widehat{s l}(2 \mid 1)$

2.1. Алгебра $\widehat{s l}(2)$.

Модули Верма, отображение спектрального потока и экстремальные диаграммы. Аффинная алгебра $\widehat{s l}(2)$ уровня $k$ задается соотношениями

$$
\begin{gathered}
{\left[J_{m}^{0}, J_{n}^{ \pm}\right]= \pm J_{m+n}^{ \pm}, \quad\left[J_{m}^{0}, J_{n}^{0}\right]=\frac{K}{2} m \delta_{m+n, 0},} \\
{\left[J_{m}^{+}, J_{n}^{-}\right]=K m \delta_{m+n, 0}+2 J_{m+n}^{0},}
\end{gathered}
$$

где $K$ - центральный элемент. Модуль Верма $\mathcal{M}_{j, k}$ порождается из вектора старшего веса $|j, k\rangle_{s l(2)}$, удовлетворяющего условиям аннигиляции

$$
\begin{gathered}
J_{\geq 0}^{+}|j, k\rangle_{s l(2)}=J_{\geq 1}^{0}|j, k\rangle_{s l(2)}=J_{\geq 1}^{-}|j, k\rangle_{s l(2)}=0, \\
J_{0}^{0}|j, k\rangle_{s l(2)}=j|j, k\rangle_{s l(2)}, \quad K|j, k\rangle_{s l(2)}=k|j, k\rangle_{s l(2)}
\end{gathered}
$$

(где, вообще говоря, $j, k \in \mathbb{C}$ ), свободным действием остальных мод операторов $J^{+}, J^{-}$ и $J^{0}$. Структуру $\widehat{s l}(2)$-модулей Верма удобно описывать с помощью әкстремальной диаграммы

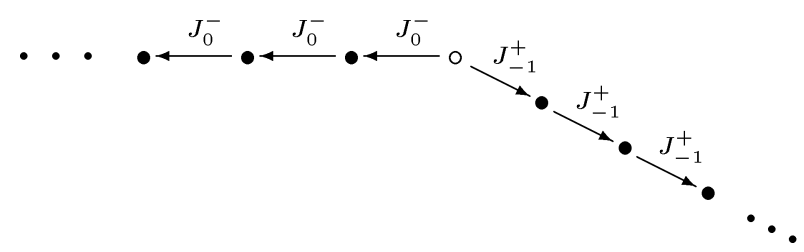

Точками здесь изображены не все состояния в модуле, а только те, которые являются граничными в следуюшем смысле. В модуле Верма имеются две $\mathbb{Z}$-градуировки, одна из которых задается оператором $J_{0}^{0}$, а другая - как (минус) сумма мод генераторов в каждом операторном мономе, примененном к вакууму (эту градуировку можно реализовать сугаваровским оператором $\left.L_{0}\right)$. Действие операторов $J_{n}^{+}$увеличивает $J_{0}^{0}$-градуировку на 1 , что изображается сдвигом вправо, градуировка же "по уровню" соответствует сдвигу вниз. Важно, что допустимые биградуировки состояний из модуля лежат внутри угла на диаграмме, в то время как в северо-восточной части плоскости состояний из модуля нет.

Автоморфизмы алгебры $\widehat{s l}(2)$ - это каноническая инволюция и отображение спектрального потока

$$
\mathcal{U}_{\theta}: \quad J_{n}^{+} \mapsto J_{n+\theta}^{+}, \quad J_{n}^{-} \mapsto J_{n-\theta}^{-}, \quad J_{n}^{0} \mapsto J_{n}^{0}+\frac{k}{2} \theta \delta_{n, 0}, \quad \theta \in \mathbb{Z} .
$$

Модули над $\widehat{s l}(2)$-алгеброй, вообще говоря, не инвариантны относительно действия спектрального потока.

ОПРЕДЕЛЕниЕ 2.1. Скрученньй модуль Верма $\mathfrak{M}_{j, k, \theta}$ свободно порожден генераторами $J_{\leq \theta-1}^{+}, J_{\leq-\theta}^{-}$и $J_{\leq-1}^{0}$ из “скрученного" вектора старшего веса $|j, k ; \theta\rangle_{s l(2)}$, определяемого условиями

$$
\begin{aligned}
J_{\geq \theta}^{+}|j, k ; \theta\rangle_{s l(2)} & =J_{\geq 1}^{0}|j, k ; \theta\rangle_{s l(2)} \\
\left(J_{0}^{0}+\frac{k}{2} \theta\right)|j, k ; \theta\rangle_{s l(2)} & =j|j, k ; \theta\rangle_{s l(2)}^{-} .
\end{aligned}
$$


Соответствуюшая экстремальная диаграмма является "врашением" (или скорее перекосом) диаграммы (2.3) с сохранением основного свойства - наличия угла, меньшего $180^{\circ}$. Отождествим $|j, k\rangle_{s l(2)}=|j, k ; 0\rangle_{s l(2)}$. Все возможные (целочисленные) скручивания модулей Верма составляют категорию $\mathcal{V} \mathcal{E} \mathcal{R}$.

\section{“Расслабленные” модули Верма.}

ОПРЕДЕлЕниЕ 2.2. Пусть $\theta \in \mathbb{Z}$. Расслабленный скрученный модуль Верма $\Re_{j, \Lambda, k ; \theta}$ порожден из вектора $|j, \Lambda, k ; \theta\rangle_{s l(2)}$, подчиненного условиям аннигилящии

$$
J_{\geq \theta+1}^{+}|j, \Lambda, k ; \theta\rangle_{s l(2)}=J_{\geq 1}^{0}|j, \Lambda, k ; \theta\rangle_{s l(2)}=J_{\geq-\theta+1}^{-}|j, \Lambda, k ; \theta\rangle_{s l(2)}=0,
$$

свободным действием операторов $J_{\leq \theta-1}^{+}, J_{\leq-\theta-1}^{-}$и $J_{\leq-1}^{0}$, а также действием операторов $J_{\theta}^{+}$и $J_{-\theta}^{-}$с условием

$$
\left(J_{-\theta}^{-} J_{\theta}^{+}+(k+2) \theta\left(j-\frac{k}{4} \theta\right)\right)|j, \Lambda, k ; \theta\rangle_{s l(2)}=\Lambda|j, \Lambda, k ; \theta\rangle_{s l(2)},
$$

кроме того,

$$
\left(J_{0}^{0}+\frac{k}{2} \theta\right)|j, \Lambda, k ; \theta\rangle_{s l(2)}=j|j, \Lambda, k ; \theta\rangle_{s l(2)} \text {. }
$$

Соответствующая экстремальная диаграмма открывается до развернутого угла; при $\theta=0$ это выглядит как

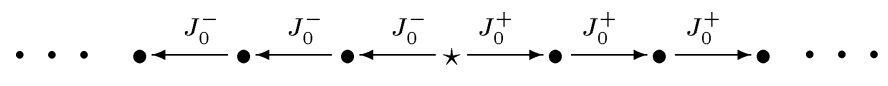

Теперь состояния из модуля имеют биградуировки, заполняюшие решетку снизу от пря-

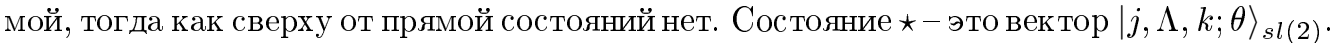
Другие состояния $|j, \Lambda, k ; \theta| n\rangle_{s l(2)}, n \in \mathbb{Z}$, из экстремальной диаграммы имеют вид

$$
|j, \Lambda, k ; \theta| n\rangle_{s l(2)}= \begin{cases}\left(J_{-\theta}^{-}\right)^{-n}|j, \Lambda, k ; \theta\rangle_{s l(2)}, & n<0, \\ \left(J_{\theta}^{+}\right)^{n}|j, \Lambda, k ; \theta\rangle_{s l(2)}, & n \geq 0 .\end{cases}
$$

Обозначим также для простоты $\left.|j, \Lambda, k| n\rangle_{s l(2)}=|j, \Lambda, k ; 0| n\right\rangle_{s l(2)}$.

В случае общего положения можно путешествовать по экстремальной диаграмме в обоих направлениях: например, диаграмма (2.9) принимает вид

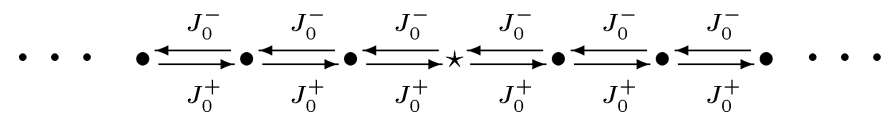

Композиция прямой и обратной стрелок в каждом случае дает оператор умножения на число, в силу чего все состояния оказываются эквивалентными. Однако эквивалентность разрушается, если это число оказывается нулем. При этом порождаются обычнье модули Верма ${ }^{2)}$. А именно, в нескрученном случае, как в диаграмме (2.9), равенство $\Lambda=n(n+1)+2 n j$ при некотором $n \leq-1$ влечет за собой $\left.J_{0}^{+}|j, \Lambda, k| n\right\rangle_{s l(2)}=0$. При этом нельзя вернуться к состоянию $\star$, применяя оператор $J_{0}^{+}$. Вместо этого мы

\footnotetext{
${ }^{2)}$ Собственно модулями Верма в $\widehat{s l}(2)$-контексте мы будем называть нерасслабленные модули.
} 
действуем первой незануляющей модой тока $J^{+}$, а именно $J_{-1}^{+}$, так что экстремальная диаграмма принимает вид

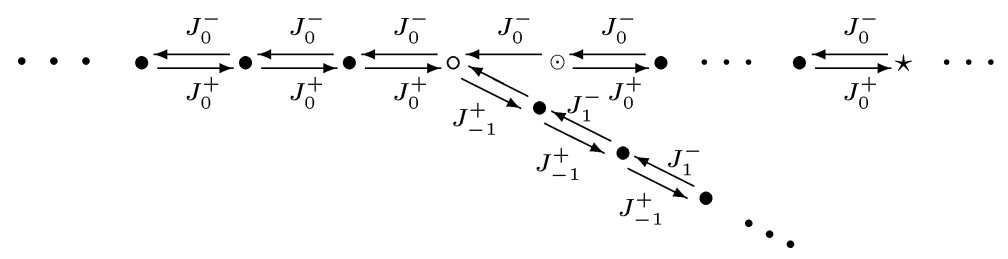

где в поддиаграмме мы узнаем экстремальную диаграмму обычного модуля Верма. Таким образом, модуль Верма можно рассматривать как подмодуль расслабленного модуля Верма.

Аналогичным образом при $n \geq 1$ имеем $\Lambda=n(n-1)+2(n-1) j \Longrightarrow$ $\left.J_{0}^{-}|j, \Lambda, k| n\right\rangle_{s l(2)}=0$, и в этом случае ветвление экстремальной диаграммы зеркально симметрично приведенному выше, а подмодуль дается образом обычного модуля Верма при отображении спектрального потока с $\theta=1$.

2.2. Алгебра $N=2$. Мы выберем базис, в котором $N=2$ суперконформная алгебра $\mathcal{A}$ задается в виде (где скобки $[$,$] обозначают суперкоммутатор)$

$$
\begin{gathered}
{\left[\mathcal{L}_{m}, \mathcal{L}_{n}\right]=(m-n) \mathcal{L}_{m+n}, \quad\left[\mathcal{H}_{m}, \mathcal{H}_{n}\right]=\frac{\mathbf{c}}{3} m \delta_{m+n, 0},} \\
{\left[\mathcal{L}_{m}, \mathcal{G}_{n}\right]=(m-n) \mathcal{G}_{m+n}, \quad\left[\mathcal{H}_{m}, \mathcal{G}_{n}\right]=\mathcal{G}_{m+n},} \\
{\left[\mathcal{L}_{m}, \mathcal{Q}_{n}\right]=-n \mathcal{Q}_{m+n}, \quad\left[\mathcal{H}_{m}, \mathcal{Q}_{n}\right]=-\mathcal{Q}_{m+n}, \quad m, n \in \mathbb{Z} .} \\
{\left[\mathcal{L}_{m}, \mathcal{H}_{n}\right]=-n \mathcal{H}_{m+n}+\frac{\mathbf{c}}{6}\left(m^{2}+m\right) \delta_{m+n, 0},} \\
{\left[\mathcal{G}_{m}, \mathcal{Q}_{n}\right]=2 \mathcal{L}_{m+n}-2 n \mathcal{H}_{m+n}+\frac{\mathbf{c}}{3}\left(m^{2}+m\right) \delta_{m+n, 0},}
\end{gathered}
$$

Генераторы $\mathcal{L}_{m}, \mathcal{Q}_{m}, \mathcal{H}_{m}$ и $\mathcal{G}_{m}$ называются генераторами Вирасоро, "БРСТ"-током, $U(1)$-током и фермионным током спина 2. Элемент с является центральным.

Определим класс топологических ${ }^{3)}$ модулей Верма над $N=2$ алгеброй; вектор старшего веса в таком модуле удовлетворяет условиям

$$
\begin{gathered}
\mathcal{Q}_{\geq 0}|h, t\rangle_{\text {top }}=\mathcal{G}_{\geq 0}|h, t\rangle_{\text {top }}=\mathcal{L}_{\geq 1}|h, t\rangle_{\text {top }}=\mathcal{H}_{\geq 1}|h, t\rangle_{\text {top }}=0, \\
\mathcal{H}_{0}|h, t\rangle_{\text {top }}=h|h, t\rangle_{\text {top }}, \quad \mathcal{L}_{0}|h, t\rangle_{\text {top }}=0, \quad \mathbf{c}|h, t\rangle_{\text {top }}=\frac{3(t-2)}{t}|h, t\rangle_{\text {top }}
\end{gathered}
$$

(последнее из которых является просто параметризацией центрального заряда через новый параметр $t$ ). Топологический модуль Верма $\mathcal{V}_{h, t}$ свободно порожден операторами $\mathcal{L}_{-m}, \mathcal{H}_{-m}, \mathcal{Q}_{-m}$ и $\mathcal{G}_{-m}$, где $m \in \mathbb{N}$, из топологического вектора старшего веса $|h, t\rangle_{\text {top }}$.

\footnotetext{
3) Название заимствовано из теории бозонной струны, где вершинные операторы материи могут быть одеты в $N=2$ примарные состояния, удовлетворяющие условиям старшего веса $(2.14)$; в этом контексте алгебра (2.13) рассматривается как топологическая.
} 
Экстремальная диаграмма топологического модуля Верма имеет вид

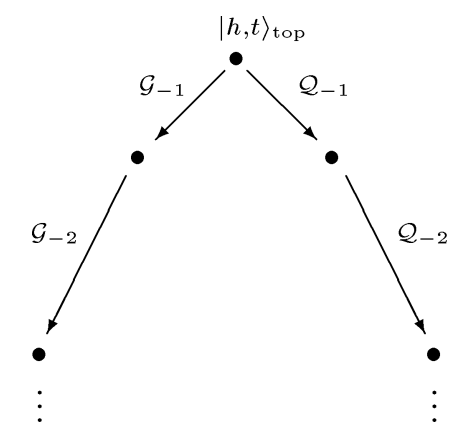

Как и в случае с $\widehat{s l}(2)$-алгеброй, подобная диаграмма описывает состояния с граничными значениями биградуировки (относительно $\left(\mathcal{H}_{0}, \mathcal{L}_{0}\right)$ ). Биградуировки всех состояний из модуля находятся во внутренней части диаграммы.

На генераторы из (2.13) спектральный поток $\mathcal{U}_{\theta}[29,37]$ действует следующим обра3ом:

$$
\begin{gathered}
\mathcal{L}_{n} \mapsto \mathcal{L}_{n}+\theta \mathcal{H}_{n}+\frac{\mathbf{c}}{6}\left(\theta^{2}+\theta\right) \delta_{n, 0}, \quad \mathcal{H}_{n} \mapsto \mathcal{H}_{n}+\frac{\mathbf{c}}{3} \theta \delta_{n, 0}, \\
\mathcal{Q}_{n} \mapsto \mathcal{Q}_{n-\theta}, \quad \mathcal{G}_{n} \mapsto \mathcal{G}_{n+\theta} .
\end{gathered}
$$

При $\theta \in \mathbb{Z}$ спектральный поток является автоморфизмом алгебры $\mathcal{A}$. Для произвольных $\theta$ мы получаем семейство алгебр, любые два члена которого $\mathcal{A}_{\theta_{1}}$ и $\mathcal{A}_{\theta_{2}}$ связаны изоморфизмом вида (2.16), в котором $\theta=\theta_{1}-\theta_{2}$. Это семейство включает, в частности, алгебры Неве-Шварца и Рамона.

Теперь мы определим скрученные топологические модули Верма $\mathfrak{V}_{h, t ; \theta}$. Такой модуль имеет вектор старшего веса, определенный соотношениями

$$
\begin{array}{rllll}
\mathcal{L}_{m}|h, t ; \theta\rangle_{\text {top }} & =0, & m \geq 1, & \mathcal{Q}_{\lambda}|h, t ; \theta\rangle_{\text {top }}=0, & \lambda \in-\theta+\mathbb{N}_{0},
\end{array} \quad \theta \in \mathbb{Z},
$$

и параметризуемый собственными значениями “картановских" генераторов как

$$
\left(\mathcal{H}_{0}+\frac{\mathbf{c}}{3} \theta\right)|h, t ; \theta\rangle_{\text {top }}=h|h, t ; \theta\rangle_{\text {top }}, \quad\left(\mathcal{L}_{0}+\theta \mathcal{H}_{0}+\frac{\mathbf{c}}{6}\left(\theta^{2}+\theta\right)\right)|h, t ; \theta\rangle_{\text {top }}=0
$$

Будем называть $|h, t ; \theta\rangle_{\text {top }}$ скрученным топологическим вектором старшего веса. Случай $\theta=0$ описывает введенные выше "обычные" топологические модули Верма: $\mathcal{V}_{h, t}=\mathfrak{V}_{h, t ; 0}$.

Введем также $N=2$ модули Верма другого типа, а именно такие, в которых вектор старшего веса удовлетворяет условиям

$$
\begin{gathered}
\mathcal{Q}_{\geq 1}|h, \ell, t\rangle=\mathcal{G}_{\geq 0}|h, \ell, t\rangle=\mathcal{L}_{\geq 1}|h, \ell, t\rangle=\mathcal{H}_{\geq 1}|h, \ell, t\rangle=0, \\
\mathcal{H}_{0}|h, \ell, t\rangle=h|h, \ell, t\rangle, \quad \mathcal{L}_{0}|h, \ell, t\rangle=\ell|h, \ell, t\rangle .
\end{gathered}
$$

Соответствующий модуль $U_{h, \ell, t}$ свободно порождается действием на $|h, \ell, t\rangle$ остальных $N=2$ генераторов. Из-за свойства иметь, вообше говоря, ненулевую размерность $\ell$ векторы $|h, \ell, t\rangle$ называются "массивными", а $U_{h, \ell, t}$ соответственно - массивным $N=2$ 
модулем. Аналог экстремальной диаграммы (2.11) для массивного модуля Верма имеет вид

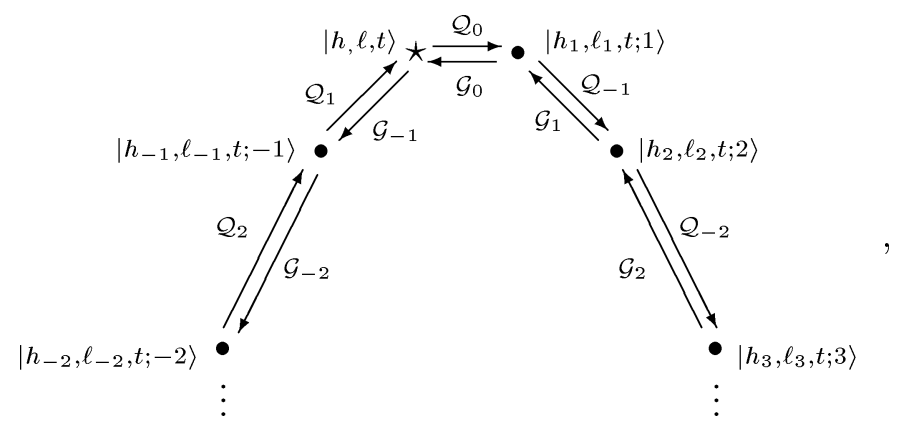

где стрелки, отображающие обратно в сторону состояния $|h, \ell, t\rangle$, описываются следуюшим образом:

$$
\begin{array}{ll}
\mathcal{Q}_{-\theta}|h, \ell, t ; \theta\rangle=2 \ell\left|h-\frac{2}{t}, \ell+h-\frac{2}{t}, t ; \theta+1\right\rangle, & \theta<0, \\
\mathcal{G}_{\theta-1}|h, \ell, t ; \theta\rangle=2(\ell-h)\left|h+\frac{2}{t}, \ell-h, t ; \theta-1\right\rangle, & \theta>0 .
\end{array}
$$

Из (2.22) следует, что, как только один из множителей в правой части обращается в нуль, соответствуюшее состояние удовлетворяет скрученным топологическим условиям старшего веса (2.14). Экстремальная диаграмма ветвится в таких “топологических точках", например

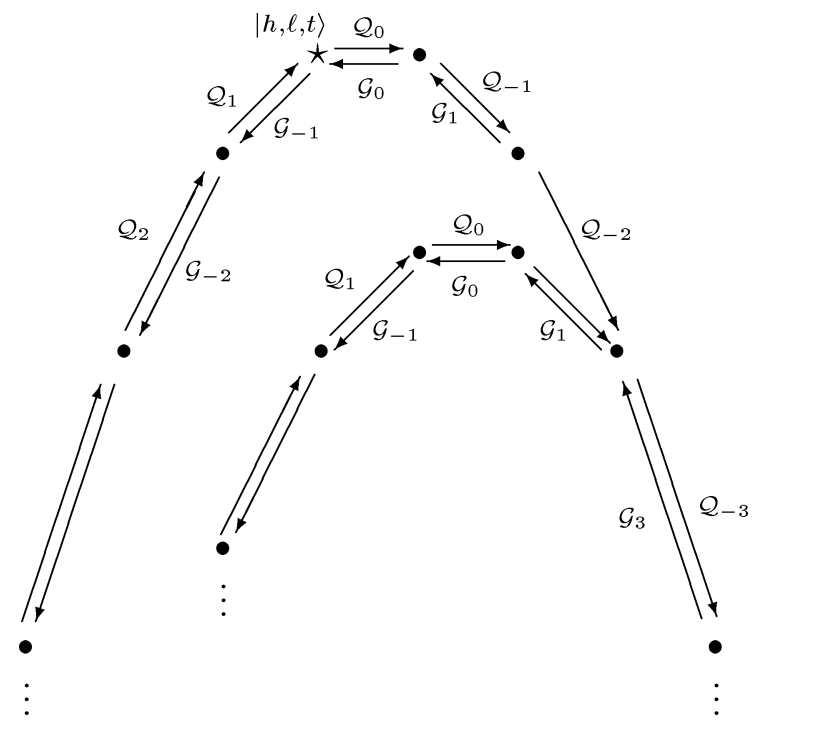

Внутренняя диаграмма здесь соответствует $N=2$ подпредставлению и оказывается экстремальной диаграммой скрученного топологического модуля Верма. (В дальнейшем мы будем для простоты заменять “дискретную аппроксимацию" парабол на экстремальных диаграммах просто параболами.) 
Скручивание массивного модуля Верма осуществляется посредством наложения скрученных условий старшего веса:

$$
\begin{aligned}
\mathcal{L}_{m}|h, \ell, t ; \theta\rangle & =0, \quad m \geq 1, \quad \mathcal{Q}_{\lambda}|h, \ell, t ; \theta\rangle=0, \quad \lambda \in-\theta+\mathbb{N}, \\
\mathcal{H}_{m}|h, \ell, t ; \theta\rangle & =0, \quad m \geq 1, \quad \mathcal{G}_{\nu}|h, \ell, t ; \theta\rangle=0, \quad \nu=\theta+\mathbb{N}_{0}, \\
\left(\mathcal{H}_{0}+\frac{\mathbf{c}}{3} \theta\right)|h, \ell, t ; \theta\rangle & =h|h, \ell, t ; \theta\rangle, \\
\left(\mathcal{L}_{0}+\theta \mathcal{H}_{0}+\frac{\mathbf{c}}{6}\left(\theta^{2}+\theta\right)\right)|h, \ell, t ; \theta\rangle & =\ell|h, \ell, t ; \theta\rangle .
\end{aligned}
$$

2.3. Алгебра $\widehat{s l}(2 \mid 1)$. Алгебра порождается четырьмя бозонными и четырьмя фермионными токами: $E^{12}, H^{-}, F^{12}, H^{+}$и $E^{1}, E^{2}, F^{1}, F^{2}$, соответственно. Ненулевые коммутационные соотношения имеют вид

$$
\begin{aligned}
{\left[H_{m}^{-}, E_{n}^{12}\right] } & =E_{m+n}^{12}, & {\left[H_{m}^{-}, F_{n}^{12}\right] } & =-F_{m+n}^{12}, \\
{\left[E_{m}^{12}, F_{n}^{12}\right] } & =m \delta_{m+n, 0} K+2 H_{m+n}^{-}, & {\left[H_{m}^{ \pm}, H_{n}^{ \pm}\right] } & =\mp \frac{1}{2} m \delta_{m+n, 0} K, \\
{\left[F_{m}^{12}, E_{n}^{2}\right] } & =F_{m+n}^{1}, & {\left[E_{m}^{12}, F_{n}^{2}\right] } & =-E_{m+n}^{1}, \\
{\left[F_{m}^{12}, E_{n}^{1}\right] } & =-F_{m+n}^{2}, & {\left[E_{m}^{12}, F_{n}^{1}\right] } & =E_{m+n}^{2}, \\
{\left[H_{m}^{ \pm}, E_{n}^{1}\right] } & =\frac{1}{2} E_{m+n}^{1}, & {\left[H_{m}^{ \pm}, F_{n}^{1}\right] } & =-\frac{1}{2} F_{m+n}^{1}, \\
{\left[H_{m}^{ \pm}, E_{n}^{2}\right] } & =\mp \frac{1}{2} E_{m+n}^{2}, & {\left[H_{m}^{ \pm}, F_{n}^{2}\right] } & = \pm \frac{1}{2} F_{m+n}^{2}, \\
{\left[E_{m}^{1}, F_{n}^{1}\right] } & =-m \delta_{m+n, 0} K+H_{m+n}^{+}-H_{m+n}^{-}, & & \\
{\left[E_{m}^{2}, F_{n}^{2}\right] } & =m \delta_{m+n, 0} K+H_{m+n}^{+}+H_{m+n}^{-}, & & \\
{\left[E_{m}^{1}, E_{n}^{2}\right] } & =E_{m+n}^{12}, & & {\left[F_{m}^{1}, F_{n}^{2}\right]=F_{m+n}^{12}, }
\end{aligned}
$$

где элемент $K$ - центральный. Как видно, $\widehat{s l}(2)$-подалгебра порождается генераторами $E_{m}^{12}, H_{m}^{-}$и $F_{m}^{12}$; коммутируюшая с ней $U(1)$-подалгебра порождена током $H_{m}^{+}$.

Алгебра $\widehat{s l}(2 \mid 1)$ допускает автоморфизм четвертого порядка (который нам не понадобится), а также инволютивный автоморфизм

$$
E_{n}^{1} \mapsto E_{n}^{2}, \quad E_{n}^{2} \mapsto E_{n}^{1}, \quad F_{n}^{1} \mapsto-F_{n}^{2}, \quad F_{n}^{2} \mapsto-F_{n}^{1}, \quad H_{n}^{+} \mapsto-H_{n}^{+}
$$

(при котором остальные генераторы остаются без изменений). Далее, отображение спектрального потока

$$
\mathcal{U}_{\theta}: \begin{aligned}
& E_{n}^{1} \mapsto E_{n-\theta}^{1}, \quad E_{n}^{2} \mapsto E_{n+\theta}^{2}, \\
& F_{n}^{1} \mapsto F_{n+\theta}^{1}, \quad F_{n}^{2} \mapsto F_{n-\theta}^{2},
\end{aligned} \quad H_{n}^{+} \mapsto H_{n}^{+}+K \theta \delta_{n, 0}
$$

(относительно которого $\widehat{s l}(2)$-подалгебра инвариантна) дает изоморфные алгебры для любых $\theta \in \mathbb{C}$. При $\theta=\frac{1}{2}$, в частности, получаем "рамоновский сектор" алгебры. Отображения спектрального потока с $\theta \in \mathbb{Z}$ являются автоморфизмами алгебры $\widehat{s l}(2 \mid 1)$. Отметим, что сушествует также еше одно независимое отображение спектрального потока, затрагиваюшее $\widehat{s l}(2)$-подалгебру. 
Скрученные условия старшего веса имеют вид

$$
\begin{gathered}
E_{\geq-\theta+\frac{1}{2}}^{1}|p, j, \kappa ; \theta\rangle=0, \quad E_{\geq \theta-\frac{1}{2}}^{2}|p, j, \kappa ; \theta\rangle=0, \quad E_{\geq 0}^{12}|p, j, \kappa ; \theta\rangle=0, \\
F_{\geq \theta+\frac{1}{2}}^{1}|p, j, \kappa ; \theta\rangle=0, \quad F_{\geq-\theta+\frac{3}{2}}^{2}|p, j, \kappa ; \theta\rangle=0, \quad F_{\geq 1}^{12}|p, j, \kappa ; \theta\rangle=0, \\
H_{0}^{+}|p, j, \kappa ; \theta\rangle=(p-\kappa \theta)|p, j, \kappa ; \theta\rangle, \quad H_{0}^{-}|p, j, \kappa ; \theta\rangle=j|p, j, \kappa ; \theta\rangle, \\
K|p, j, \kappa ; \theta\rangle=\kappa|p, j, \kappa ; \theta\rangle, \\
H_{\geq 1}^{+}|p, j, \kappa ; \theta\rangle=0, \quad H_{\geq 1}^{-}|p, j, \kappa ; \theta\rangle=0 .
\end{gathered}
$$

"Обычный" вектор старшего веса мы будем обозначать как $|p, j, \kappa\rangle \equiv|p, j, \kappa ; 0\rangle$.

Если $-p \pm j+\frac{\kappa}{2}-r(\kappa+1) \neq 0, r \in \mathbb{Z}$, то условия старшего веса (2.28) эквивалентны при всех $\theta \in \mathbb{Z}$. Действительно, применяя моды фермионных генераторов, мы строим экстремальные состояния

$$
|p+(\kappa+1) r, j, \kappa ; r\rangle=\left\{\begin{array}{cc}
E_{r-\frac{1}{2}}^{2} \ldots E_{-\frac{3}{2}}^{2} \cdot F_{r+\frac{1}{2}}^{1} \ldots F_{-\frac{1}{2}}^{1}|p, j, \kappa\rangle, \quad r \leq 0 \\
E_{-r+\frac{1}{2}}^{1} \ldots E_{-\frac{1}{2}}^{1} \cdot F_{-r+\frac{3}{2}}^{2} \ldots F_{\frac{1}{2}}^{2}|p, j, \kappa\rangle, \quad r \geq 1
\end{array}\right.
$$

которые удовлетворяют в точности скрученным условиям старшего веса $(2.28)$ с $\theta=r$. Движение по экстремальным состояниям в обратную сторону описывается следующим образом:

$$
\begin{aligned}
& E_{\frac{1}{2}}^{1} \ldots E_{-r-\frac{1}{2}}^{1} F_{\frac{3}{2}}^{2} \ldots F_{-r+\frac{1}{2}}^{2}|p+(\kappa+1) r, j, \kappa ; r\rangle= \\
& \quad=\prod_{i=r}^{-1}\left(i(\kappa+1)+j+p-\frac{\kappa}{2}\right)\left((i+1)(\kappa+1)-j+p-\frac{\kappa}{2}\right)|p, j, \kappa\rangle, \quad r \leq 0, \\
& E_{-\frac{1}{2}}^{2} \ldots E_{r-\frac{3}{2}}^{2} F_{\frac{1}{2}}^{1} \ldots F_{r-\frac{1}{2}}^{1}|p+(\kappa+1) r, j, \kappa ; r\rangle= \\
& \quad=\prod_{i=1}^{r}\left((i-1)(\kappa+1)+j+p-\frac{\kappa}{2}\right)\left(i(\kappa+1)-j+p-\frac{\kappa}{2}\right)|p, j, \kappa\rangle, \quad r \geq 1 .
\end{aligned}
$$

Поэтому, пока ни один из множителей в (2.30) не обращается в нуль, мы имеем экстремальную диаграмму

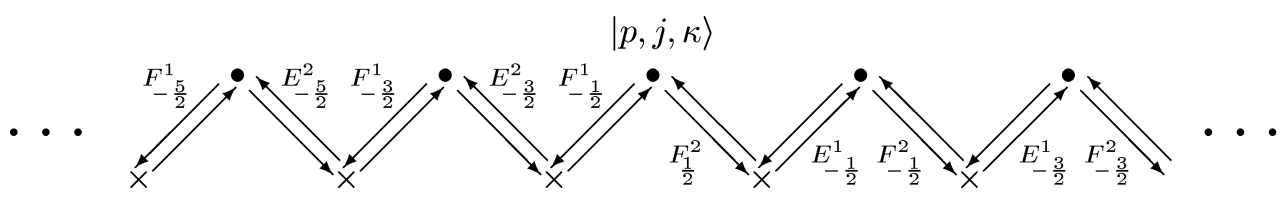

Для алгебры $\widehat{s l}(2 \mid 1)$ имеются три $\mathbb{Z}$-градуировки, только две из которых (по $H_{0}^{+}$и $\left.H_{0}^{-}\right)$ показаны в плоскости диаграммы, а изменение градуировки по уровню происходит в перпендикулярном направлении. Жирные точки обозначают состояния (2.29), перечисляемые параметром $\theta \in \mathbb{Z}$, а крестики удовлетворяют модифицированным условиям старшего веса

$$
\begin{aligned}
& E_{\geq-(\theta-1)+\frac{1}{2}}^{1} \approx 0, \quad E_{\geq(\theta-1)+\frac{1}{2}}^{2} \approx 0, \quad E_{\geq 0}^{12} \approx 0, \quad H_{\geq 1}^{+} \approx 0, \\
& F_{\geq(\theta-1)+\frac{1}{2}}^{1} \approx 0, \quad F_{\geq-(\theta-1)+\frac{1}{2}}^{2} \approx 0, \quad F_{\geq 1}^{12} \approx 0, \quad H_{\geq 1}^{-} \approx 0,
\end{aligned}
$$


которые эквивалентны стандартным, если $p-j-\frac{1}{2} \kappa \neq 0$. Как и для двух рассмотренных выше алгебр, свойство “транзитивности” нарушается, когда генератор, который, вообше говоря, отображает в соседнее состояние, оказывается оператором уничтожения из-за того, что соответствующий множитель обращается в нуль.

\section{3. КОНСТРУКЦИИ СИНГУЛЯРНЫХ ВЕКТОРОВ}

\section{1. Сингулярные векторы алгебры $\widehat{s l}(2)$.}

Сингулярные векторы в обычных $\widehat{s l}(2)$-модулях Верма. Сингулярный вектор в модуле Верма $\mathcal{M}_{j, k}$ определяется как вектор, не пропорциональный вектору старшего веса, который тем не менее удовлетворяет тем же условиям обрашения в нуль (2.2), что и вектор старшего веса.

Напомним известную конструкцию для сингулярных векторов [2]. Вводятся объекты $\left(J_{-1}^{+}\right)^{\alpha}$ и $\left(J_{0}^{-}\right)^{\alpha}$, которые реализуют стандартное действие генераторов аффинной группы Вейля на пространстве старших весов. А именно, $\left(J_{-1}^{+}\right)^{\alpha}$ и $\left(J_{0}^{-}\right)^{\alpha}$ следуюшим образом действуют на линии $k=$ const в плоскости $k j$ старших весов:

$$
\begin{aligned}
\left(J_{0}^{-}\right)^{2 j+1}:|j, k\rangle_{s l(2)} & \rightarrow|-1-j, k\rangle_{s l(2)}, \\
\left(J_{-1}^{+}\right)^{k+1-2 j}:|j, k\rangle_{s l(2)} & \rightarrow|k+1-j, k\rangle_{s l(2)} .
\end{aligned}
$$

Это действие можно продолжить с множества векторов старшего веса на модули Верма над этими векторами, используя формулы

$$
\begin{aligned}
\left(J_{0}^{-}\right)^{\alpha} J_{m}^{+}= & -\alpha(\alpha-1) J_{m}^{-}\left(J_{0}^{-}\right)^{\alpha-2}-2 \alpha J_{m}^{0}\left(J_{0}^{-}\right)^{\alpha-1}+J_{m}^{+}\left(J_{0}^{-}\right)^{\alpha} \\
\left(J_{0}^{-}\right)^{\alpha} J_{m}^{0}= & \alpha J_{m}^{-}\left(J_{0}^{-}\right)^{\alpha-1}+J_{m}^{0}\left(J_{0}^{-}\right)^{\alpha} \\
\left(J_{-1}^{+}\right)^{\alpha} J_{m}^{-}= & -\alpha(\alpha-1) J_{m-2}^{+}\left(J_{-1}^{+}\right)^{\alpha-2}-k \alpha \delta_{m-1,0}\left(J_{-1}^{+}\right)^{\alpha-1}+ \\
& +2 \alpha J_{m-1}^{0}\left(J_{-1}^{+}\right)^{\alpha-1}+J_{m}^{-}\left(J_{-1}^{+}\right)^{\alpha} \\
\left(J_{-1}^{+}\right)^{\alpha} J_{m}^{0}= & -\alpha J_{m-1}^{+}\left(J_{-1}^{+}\right)^{\alpha-1}+J_{m}^{0}\left(J_{-1}^{+}\right)^{\alpha}
\end{aligned}
$$

Тогда имеем

Teopema 3.1.

I ([1]). Сингулярный вектор существует в $\mathcal{M}_{j, k}$, если и только если $j=\mathbf{j}^{+}(r, s, k)$ uли $j=\mathbf{j}^{-}(r, s, k)$, əде

$$
\begin{aligned}
& \mathbf{j}^{+}(r, s, k)=\frac{r-1}{2}-(k+2) \frac{s-1}{2}, \\
& \mathbf{j}^{-}(r, s, k)=-\frac{r+1}{2}+(k+2) \frac{s}{2},
\end{aligned}
$$

II ([2]). Все сингулярные векторы $\left|\mathrm{MFF}^{ \pm}(r, s, k)\right\rangle$ в $\widehat{s l}(2)$-модуле Верма $\mathcal{M}_{\mathbf{j}^{ \pm}(r, s, k), k}$ даются формулами

$$
\begin{aligned}
\left|\mathrm{MFF}^{+}(r, s, k)\right\rangle=( & \left.J_{0}^{-}\right)^{r+(s-1)(k+2)}\left(J_{-1}^{+}\right)^{r+(s-2)(k+2)} \times \cdots \\
& \cdots \times\left(J_{0}^{-}\right)^{r-(s-1)(k+2)}\left|\mathbf{j}^{+}(r, s, k), k\right\rangle_{\widehat{s l}(2)}, \\
\left|\mathrm{MFF}^{-}(r, s, k)\right\rangle=( & \left.J_{-1}^{+}\right)^{r+(s-1)(k+2)}\left(J_{0}^{-}\right)^{r+(s-2)(k+2)} \times \cdots \\
& \cdots \times\left(J_{-1}^{+}\right)^{r-(s-1)(k+2)}\left|\mathbf{j}^{-}(r, s, k), k\right\rangle_{\widehat{s l}(2)}
\end{aligned}
$$


Показатели степеней в формулах (3.1) получены из требования, чтобы $\left(J_{-1}^{+}\right)^{\alpha}$ и $\left(J_{0}^{-}\right)^{\alpha}$ сохраняли условия старшего веса $(2.2)$, что является основным принципом при построении "продолженных" формул типа (3.4), (3.5): скрученные условия старшего веса должны формально выполняться при соответствуюших комплексных $\theta$ для мономов, получаемых из (3.4), (3.5) отбрасыванием слева нескольких сомножителей (если под сомножителями понимаются указанные в формуле степени генераторов).

Сингулярные векторы в скрученных модулях $\mathfrak{M}_{j, k ; \theta}$ получаются из $(3.4),(3.5)$ после применения отображения спектрального потока.

Сингулярные векторы в расслабленных $\widehat{s l}(2)$-модулях Верма. Мы рассмотрим сингулярные векторы в расслабленных модулях Верма только в "нескрученном" случае $\theta=0$, аналогичные результаты для случая $\theta \neq 0$ получаются применением спектрального потока.

Сингулярные векторы в расслабленных модулях Верма естественным образом разбиваются на две группы: те, которые лежат на верхнем этаже экстремальной диаграммы, и те, которые лежат ниже верхнего этажа. Первые - "заряженные" сингулярные векторы - непосредственно следуют из анализа экстремальных диаграмм. Это просто точки, из которых растут обычные модули Верма. А именно, при

$$
\Lambda_{\mathrm{ch}}(n, j)= \begin{cases}n(n+1)+2 n j, & n \leq-1 \\ n(n-1)+2(n-1) j, & n \geq 1\end{cases}
$$

состояния

$$
|C(p, j, k)\rangle_{s l(2)}= \begin{cases}\left(J_{0}^{-}\right)^{-p}\left|j, \Lambda_{\mathrm{ch}}(p, j), k\right\rangle_{s l(2)}, & p \leq-1, \\ \left(J_{0}^{+}\right)^{p+1}\left|j, \Lambda_{\mathrm{ch}}(p, j), k\right\rangle_{s l(2)}, & p \geq 0,\end{cases}
$$

удовлетворяют вермовским условиям старшего веса, если $p \leq-1$, и скрученным условиям Верма с параметром скручивания $\theta=1$, если $p \geq 1$.

Теперь обратимся к "расслабленным" сингулярным векторам, т.е. к тем, которые порождают расслабленные подмодули Верма в расслабленном модуле Верма.

Tеорема 3.2. Расслабленные сингулярные векторы в расслабленном модуле Верма $\mathcal{R}_{j, \Lambda, k}$ существуют тогда и только тогда, когда $\Lambda=\Lambda(r, s, j, k)$, где

$$
\Lambda(r, s, j, k)=-\left(j-\mathbf{j}^{-}(r, s, k)\right)\left(j-\mathbf{j}^{+}(r, s+1, k)\right) .
$$

В случае общего положения расслабленный сингулярный вектор в $\mathcal{R}_{j, \Lambda}(r, s, j, k), k$ имеет представителей

$$
\begin{aligned}
\left|\Sigma^{-}(r, s, j, k)\right\rangle= & \left(J_{0}^{-}\right)^{\mathbf{j}^{-}(r, s, k)-j+r+r s} \mathcal{M F F}^{-}(r, s, k) \times \\
& \times\left(J_{0}^{-}\right)^{-\mathbf{j}^{-}(r, s, k)+j}|j, \Lambda(r, s, j, k), k\rangle_{s l(2)}
\end{aligned}
$$

$u$

$$
\begin{aligned}
\left|\Sigma^{+}(r, s, j, k)\right\rangle= & \left(J_{0}^{+}\right)^{-1-\mathbf{j}^{+}(r, s+1, k)+j+r+r s} \mathcal{M F F}^{+, 1}(r, s, k) \times \\
& \times\left(J_{0}^{+}\right)^{1+\mathbf{j}^{+}(r, s+1, k)-j}|j, \Lambda(r, s, j, k), k\rangle_{s l(2)}
\end{aligned}
$$


(әде $\mathcal{M F F}^{+, \theta}$ есть отобрахсение оператора сингулярного вектора $\mathcal{M F F}^{+}$под действием спектрального потока (2.4)). Состояния (3.9) и (3.10) являются элементами расслабленного модуля Верма $\mathcal{R}_{j, \Lambda(r, s, j, k), k}$ и удовлетворяют расслабленным условиям старшего веса

$$
\begin{gathered}
J_{\geq 1}^{+}\left|\Sigma^{ \pm}(r, s, j, k)\right\rangle=J_{\geq 1}^{0}\left|\Sigma^{ \pm}(r, s, j, k)\right\rangle=J_{\geq 1}^{-}\left|\Sigma^{ \pm}(r, s, j, k)\right\rangle=0 \\
J_{0}^{-} J_{0}^{+}\left|\Sigma^{ \pm}(r, s, j, k)\right\rangle=\Lambda^{ \pm}(r, s, j, k)\left|\Sigma^{ \pm}(r, s, j, k)\right\rangle \\
J_{0}^{0}\left|\Sigma^{ \pm}(r, s, j, k)\right\rangle=(j \pm r s)\left|\Sigma^{ \pm}(r, s, j, k)\right\rangle
\end{gathered}
$$

əдe $\Lambda^{ \pm}(r, s, j, k)=\frac{1}{4}(r+(k+2) s-2 r s \mp(1+2 j))(r+(k+2) s+2 r s \pm(1+2 j))$.

Таким образом, в то время как в случае общего положения модуль $\mathcal{R}_{j, \Lambda, k}$ неприводим, сингулярные векторы появляются в коразмерности 1 , а именно, когда $j, \Lambda$ и $k$ связаны одним соотношением. В общей ситуации в коразмерности 1 параметры $j$ и $k$ произвольны.

Каждое из состояний $\Sigma^{ \pm}(r, s, j, k)$ порождает весь расслабленный подмодуль Верма; в частности, $J_{0}^{ \pm}$-потомки векторов $(3.9)$ и (3.10) заполняют одну и ту же экстремальную поддиаграмму и совпадают с точностью до множителя, когда они оказываются в одной и той же градуировке:

$$
\begin{aligned}
c_{+}(i, j, k)\left(J_{0}^{+}\right)^{i}\left|\Sigma^{-}(r, s, j, k)\right\rangle & =c_{-}(i, j, k)\left(J_{0}^{-}\right)^{2 r s-i}\left|\Sigma^{+}(r, s, j, k)\right\rangle, & & 0 \leq i \leq 2 r s, \\
c_{+}(i, j, k)\left(J_{0}^{+}\right)^{i}\left|\Sigma^{-}(r, s, j, k)\right\rangle & =c_{-}(i, j, k)\left(J_{0}^{+}\right)^{i-2 r s}\left|\Sigma^{+}(r, s, j, k)\right\rangle, & & i>2 r s, \\
c_{+}(i, j, k)\left(J_{0}^{-}\right)^{-i}\left|\Sigma^{-}(r, s, j, k)\right\rangle & =c_{-}(i, j, k)\left(J_{0}^{-}\right)^{-i+2 r s}\left|\Sigma^{+}(r, s, j, k)\right\rangle, & & i<0,
\end{aligned}
$$

где коэффициенты $c_{ \pm}(i, j, k)$ представляют собой (зависяшие от $r$ и $s$ ) полиномы по $j$ и $k$.

Однако в коразмерности больше 1 некоторые из состояний в (3.12) могут обратиться в нуль. Например, может оказаться невозможным получить $\Sigma^{+}$-представитель сингулярного вектора как $J_{0}^{+}$-потомок вектора $\Sigma^{-}$и/или получить $\Sigma^{-}$как $J_{0}^{-}$-потомок вектора $\Sigma^{+}$. Мы начнем анализ этих случаев с коразмерности 2.

КоРАЗмеРность 2. Сделаем сначала следуюшее полезное для дальнейшего замечание.

Некоторые из $J_{0}^{ \pm}$-потомков вектора $\Sigma^{-}$и/или $\Sigma^{+}$могут обрашаться в нуль, если в экстремальной диаграмме подмодуля находится заряженный сингулярный вектор (так что $\Sigma^{-}$и/или $\Sigma^{+}$не будет порождать всех состояний в экстремальной диаграмме подмодуля). Чтобы увидеть, когда это происходит, надо положить $\Lambda^{ \pm}(r, s, j, k)$ в приведенных выше формулах равным значению $\Lambda_{\mathrm{ch}}(N, j \pm r s)$ (см. (3.6)), при котором сушествует заряженный сингулярный вектор.

Лемма 3.3. Пусть в расслабленном модуле Верма $\mathcal{R}_{j, \Lambda, k}$ имеется расслабленный подмодуль Верма $\mathcal{R}^{\prime}$, соответствующий расслабленному сингулярному вектору. Тогда в $\mathcal{R}^{\prime}$ существует заряэсенный сингулярный вектор, если и только если заряженный сингулярный вектор существует в модуле $\mathcal{R}_{j, \Lambda, k}$. Eсли тогда $\mathcal{C}$ - подмодуль, ассоциированный с заряженным сингулярным вектором в $R_{j, \Lambda, k}$, то заряженный сингулярный вектор в $\mathcal{R}^{\prime}$ совпадает с МФФ-сингулярным вектором в $\mathcal{C}$.

В коразмерности 2 имеются три случая сосуществования двух сингулярных векторов. Рассмотрим сначала одновременное появление двух различных расслабленных сингулярных векторов. 
ТЕОРема 3.4. Пусть $j$ находится в общем положении и $k=\frac{p}{q}$ рационально. Тогда сингулярные векторы в $\mathcal{R}_{j, \Lambda}\left(r, s, j, \frac{p}{q}\right), \frac{p}{q}$ находятся в $1: 1$-соответствии с (МФФ) сингулярными векторами в модуле Верма $\mathcal{M}_{\mathbf{j}^{-}\left(r, s, \frac{p}{q}\right), \frac{p}{q}}$, так что структура расслабленного модуля Верма $\mathcal{R}_{j, \Lambda}\left(r, s, j, \frac{p}{q}\right), \frac{p}{q}$ повторяет структуру обично-

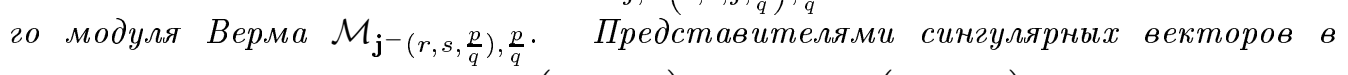

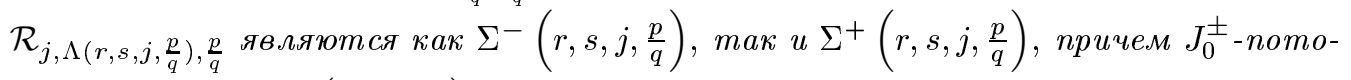
мки векторов $\Sigma^{ \pm}\left(r, s, j, \frac{p}{q}\right)$ порожсдают одну и ту же экстремальную поддиа-
грамму.

Под относительныц.м зарядом состояния $|v\rangle \in \mathcal{R}_{j \Lambda, k}$ мы будем понимать $x=y-j$, где $J_{0}^{0}|v\rangle=y|v\rangle$ (и $\left.J_{0}^{0}|j, \Lambda, k\rangle=j|j, \Lambda, k\rangle\right)$. Тогда состояния с отрицательным (положительным) относительным зарядом будут слева (соответственно, справа) от вектора $\star=|j, \Lambda, k\rangle$ в (2.9).

Еще одно полезное понятие вводится следуюшим образом. Когда в расслабленном модуле существуют два заряженных сингулярных вектора, факторизация по обоим оставляет конечномерное представление алгебры $Л u s l(2)$, реализованное на оставшихся состояниях в верхнем этаже экстремальной диаграммы. Оно будет называться осmaточны.м $\operatorname{sl}(2)$-представлением расслабленного модуля Верма.

Обратимся к структуре формул (3.9) и (3.10). В них операторы $\mathcal{M F \mathcal { F }}$ - и $\mathcal{M} \mathcal{F} \mathcal{F}^{+, 1}$ формально применяются к состояниям, вообще говоря, не лежашим в модуле $\mathcal{R}_{j, \Lambda(r, s, j, k), k}$. Однако стоящие слева от операторов $\mathcal{M F F}^{-}$и $\mathcal{M F \mathcal { F }}^{+}$ комплексные степени генераторов осушествляют отображение обратно в расслабленный модуль Верма $\mathcal{R}_{j, \Lambda}(r, s, j, k), k$. Использование формул типа (3.2) позволяет во всех случаях вычислить векторы (3.9) и (3.10) как элементы модуля $\mathcal{R}_{j, \Lambda(r, s, j, k), k}$. Однако отдельного внимания заслуживает случай, когда оба состояния $\left(J_{0}^{-}\right)^{-\mathbf{j}^{-}(r, s, k)+j}|j, \Lambda(r, s, j, k), k\rangle_{s l(2)}$ из (3.9) и $\left(J_{0}^{+}\right)^{1+\mathbf{j}^{+}(r, s+1, k)-j}|j, \Lambda(r, s, j, k), k\rangle_{s l(2)}$ из $(3.10)$ в действительности лежат в $\mathcal{R}_{j, \Lambda}(r, s, j, k), k$. При этом имеем $j=j_{\text {сс }}(n, m)$ и $\Lambda=\Lambda_{\text {сс }}(n, m)$, где

$$
j_{\mathrm{cc}}(n, m)=-\frac{1}{2}(1+m+n), \quad \Lambda_{\mathrm{cc}}(n, m)=-m n, \quad m, n \in \mathbb{Z}, \quad m \neq n, \quad k \in \mathbb{C} .
$$

Теорема 3.5. Расслабленный модуль Верма $\mathcal{R}_{j, \Lambda, k}$ содержит два заряжсенных сингулярных вектора, если и только если $j=j_{\mathrm{cc}}(n, m)$ и $\Lambda=\Lambda_{\mathrm{cc}}(n, m), \quad n, m \in \mathbb{Z}$. Более того, для модуля $\mathcal{R}_{j_{\mathrm{cc}}(n, m), \Lambda_{\mathrm{cc}}(n, m), k}$ :

1) случай $\operatorname{sign} m=\operatorname{sign} n$ является тривиальным подслучаем теоремы 3.6;

2) при $m \in \mathbb{N}_{0} \quad u-n \in \mathbb{N}$ заряженный сингулярный вектор $C(m, j, k)$ лежит справа от $|j, \Lambda, k\rangle_{s l(2)}$, а заряженный сингулярный вектор $C(n, j, k)$ - слева om $|j, \Lambda, k\rangle_{\text {sl(2) }}$.

Начиная с этого момента мы будем предполагать для определенности $m \in \mathbb{N}_{0}$ и $-n \in \mathbb{N}$; случай $n \in \mathbb{N}_{0}$ и $-m \in \mathbb{N}$ получается переобозначениями.

Третья возможность в коразмерности 2 состоит в одновременном сушествовании расслабленного и заряженного сингулярных векторов, причем в модуле Верма, построенном на заряженном сингулярном векторе, существует подмодуль Верма $\mathcal{C}^{\prime}$, экстремальная диаграмма которого частично перекрывается с экстремальной диаграммой расслабленного подмодуля.

2 Теоретическая и математическая физика, т. 112, № 2, 1997 г. 
Как видно из (3.11), $\Sigma^{-}$-представитель расслабленного сингулярного вектора находится на расстоянии в $r s$ стрелок налево от центра $\star$ экстремальной диаграммы, а $\Sigma^{+}{ }_{-}$ на таком же расстоянии справа. Может случиться, что $\Sigma^{-}$будет лежать внутри подмодуля Верма $\mathcal{C}^{\prime}$. Тогда не вся экстремальная диаграмма подмодуля будет порождаться $J_{0}^{ \pm}$-потомками вектора $\Sigma^{-}$. Однако пока не выполнены более специальные условия (коразмерность 3), вся экстремальная диаграмма расслабленного подмодуля будет тем не менее порождаться из $\Sigma^{+}$. Мы будем для краткости говорить, что состояние старшего веса допускает сингулярный вектор, если этот сингулярньй вектор сушествует в модуле, построенном на данном состоянии старшего веса.

ТеОрема 3.6. Расслабленный модуль Верма $\mathcal{R}_{j, \Lambda, k}$ содержсит заряженный и массивный сингулярные векторы, если и только если $j=j_{\mathrm{rc}}^{ \pm}(r, s, n, k)$, $\Lambda=\Lambda_{\mathrm{rc}}^{ \pm}(r, s, n, k)$, əде

$$
\begin{gathered}
j_{\mathrm{rc}}^{ \pm}(r, s, n, k)=-n-\frac{1}{2} \pm \frac{1}{2}(r-(k+2) s), \\
\Lambda_{\mathrm{rc}}^{ \pm}(r, s, n, k)=-n^{2} \pm n(r-(k+2) s), \quad n \in \mathbb{Z}, \quad r, s \in \mathbb{N} .
\end{gathered}
$$

Более того, в расслабленном модуле Верма $\mathcal{R}_{j_{\mathrm{rc}}^{ \pm}}(r, s, n, k), \Lambda_{\mathrm{rc}}^{ \pm}(r, s, n, k), k$ имеем:

I. Eсли $|n| \leq r(s+1)$, то сингулярный вектор $\left|\Sigma^{-}\left(r, s, j_{\mathrm{rc}}^{ \pm}(r, s, n, k), k\right)\right\rangle$ лежит в подмодуле Верма $\mathcal{C}^{\prime}$, вектор старшего веса которого равен

$$
|\mathrm{mff}\rangle^{ \pm}=\mathcal{M} \mathcal{F} \mathcal{F}^{ \pm}(r, s, k)\left(J_{0}^{-}\right)^{n}\left|j_{\mathrm{rc}}^{ \pm}(r, s, n, k), \Lambda_{\mathrm{rc}}^{ \pm}(r, s, n, k), k\right\rangle_{s l(2)},
$$

и представляет собой вложение МФФ сингулярного вектора $\mathrm{MFF}^{-}(r, s, k)$. При этом

$$
\left|\Sigma^{-}\left(r, s, j_{\mathrm{rc}}^{ \pm}(r, s, n, k), k\right)\right\rangle=\left(J_{0}^{-}\right)^{r+r s-n}|\mathrm{mff}\rangle^{ \pm} .
$$

II. Если $|n|>r(s+1)$ и $k$ в общем положсении, то сингулярный вектор $\left|\Sigma^{-}\left(r, s, j_{\mathrm{rc}}^{ \pm}(r, s, n, k), k\right)\right\rangle$ порождает всю экстремальную поддиаграмму и тем самым весь расслабленный подмодуль Верма.

Относительные положения $\Sigma^{-}$и $\Sigma^{+}$на экстремальной диаграмме таковы:

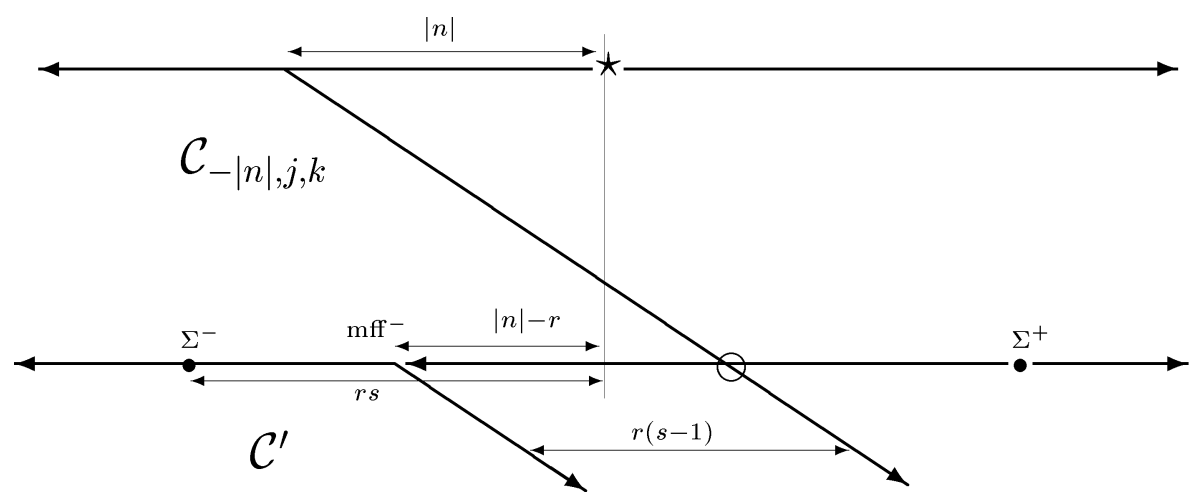

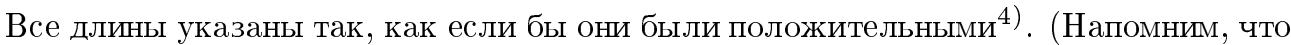
мы выбрали $n \in-\mathbb{N}$; все утверждения переформулируются для случая $n \in \mathbb{N}_{0}$ путем

\footnotetext{
${ }^{4)} \mathrm{B}$ точке, обведенной кружком, две линии не "пересекаются", т.е. на них находятся разные состояния. Это же относится и к приводимым ниже диаграммам, но больше не будет явно отмечаться. Вообще, экстремальная диаграмма не описывает $1: 1$-соответствия между точками на решетке и состояниями в модуле, такое соответствие имеется только для собственно экстремальных состояний.
} 
замен $\Sigma^{+} \leftrightarrow \Sigma^{-}$и $\mathrm{MFF}^{+} \leftrightarrow \mathrm{MFF}^{-}$.) В случае общего положения при условиях теоремы вектор $\Sigma^{+}$по-прежнему имеет бесконечно много $J_{0}^{-}-$и $J_{0}^{+}$- потомков (среди первых находится, в частности, вектор $\Sigma^{-}$).

Мы закончили анализ случая коразмерности 2. Однако для дальнейших рассмотрений в коразмерности 3 будет полезен ряд более тонких свойств выбранных представителей для сингулярных векторов. Хотя потомки вектора $\Sigma^{-}$могут не покрывать часть экстремальной подлиаграммы, тем не менее в ряде случаев весь “этаж”, соответствующий подмодулю, может быть получен из $\Sigma^{-}$с помощю следующего приема.

Заметим, что когда $\Sigma^{-}$лежит в подмодуле Верма $\mathcal{C}^{\prime}$, порожденном вектором $\mathrm{MFF}^{-}(r, s, k)$, и, таким образом, является $J_{0}^{-}$-потомком (вложения) МФФ-сингулярного вектора, это вложение МФФ-вектора может быть выбрано в качестве представителя расслабленного сингулярного вектора. Вместо (3.16) мы выбираем представителя

$$
\left.\left|\widehat{\Sigma}^{-}\left(r, s, j_{\mathrm{rc}}^{-}(r, s, n, k), k\right)\right\rangle=\mathcal{M F \mathcal { F }}^{-}(r, s, k)\left|j_{\mathrm{rc}}^{-}(r, s, n, k), \Lambda_{\mathrm{rc}}^{-}(r, s, n, k), k\right| n\right\rangle_{s l(2)}
$$

$\left(J_{0}^{-}\right.$-потомком которого является $\left.\Sigma^{-}\right)$. Это наиболее удаленное направо состояние на экстремальной диаграмме, которое можно получить из сингулярного вектора $\left|\Sigma^{-}\left(r, s, j_{\mathrm{rc}}^{-}(r, s, n, k), k\right)\right\rangle$ действием оператора $J_{0}^{+}$. Чтобы продвинуться по экстремальной диаграмме дальше направо - за пределы подмодуля Верма $\mathcal{C}^{\prime}$ - можно использовать оператор $\left(J_{0}^{-}\right)^{-1}$, которьй понимается как один из “продолженных" операторов из формул (3.1)-(3.2):

$$
\left|\widetilde{\Sigma}^{-}\left(r, s, j_{\mathrm{rc}}^{-}(r, s, n, k), k\right)\right\rangle=\left(J_{0}^{-}\right)^{-1}\left|\widehat{\Sigma}^{-}\left(r, s, j_{\mathrm{rc}}^{-}(r, s, n, k), k\right)\right\rangle .
$$

Условия, обеспечивающие существование вектора $\left|\widetilde{\Sigma}^{-}\left(r, s, j_{\mathrm{rc}}^{-}(r, s, n, k), k\right)\right\rangle$, описываются в следуюшей лемме. Определим сначала

$$
f(r, s, q, k)= \begin{cases}\prod_{m=0}^{2 r-q}(r-m-q-(k+2) s), & 1 \leq q \leq 2 r, \\ 1, & q \geq 2 r+1 .\end{cases}
$$

Лемма 3.7. Пусть $|n| \leq r(s+1)$. Тогда:

I. Eсли $f(r, s,|n|, k) \neq 0$, то вектор

$$
\begin{aligned}
& \left|\widetilde{\Sigma}^{-}\left(r, s, j_{\mathrm{rc}}^{-}(r, s, n, k), k\right)\right\rangle= \\
& \left.\quad=\left(J_{0}^{-}\right)^{-1} \mathcal{M} \mathcal{F F}^{-}(r, s, k)\left|j_{\mathrm{rc}}^{-}(r, s, n, k), \Lambda_{\mathrm{rc}}^{-}(r, s, n, k), k\right| n\right\rangle_{s l(2)}
\end{aligned}
$$

существует в расслабленном модуле Верма $\mathcal{R}_{j_{\mathrm{rc}}^{-}(r, s, n, k), \Lambda_{\mathrm{rc}}^{-}(r, s, n, k), k}$ и является представителем расслабленного сингулярного вектора.

II. При условиях теоремы $3.6 J_{0}^{+}$-потомок состояния $\left|\widetilde{\Sigma}^{-}\left(r, s, j_{\mathrm{rc}}^{-}(r, s, n, k), k\right)\right\rangle$ пропоричонален вектору (3.21):

$$
\left(J_{0}^{-}\right)^{r s-n-r-1}\left|\Sigma^{+}\left(r, s, j_{\mathrm{rc}}^{-}(r, s, n, k), k\right)\right\rangle=(-1)^{r} a(r, s, n, k)\left|\widetilde{\Sigma}^{-}\left(r, s, j_{\mathrm{rc}}^{-}(r, s, n, k), k\right)\right\rangle,
$$

$2 \partial e$

$$
a(r, s, n, k)=\prod_{m=0}^{r(s+1)-1}(m-n-r+(k+2) s) \prod_{m=0}^{r(s-1)-1}(n-m) .
$$

Таким образом, если состояние (3.19) сушествует, оно пропорционально $J_{0}^{-}$-потомку вектора $\Sigma^{+}$. 
В соответствии с этой леммой естественно дать следующее определение: обобщенным $J_{0}^{+}$-потомком (соответственно обобшенным $J_{0}^{-}$-потомком) состояния $|v\rangle \in \mathcal{R}$ называется всякое состояние в $\mathcal{R}$, которое может быть получено из $|v\rangle$ посредством произвольного числа следующих шагов:

а) действием оператором $J_{0}^{+}$(соответственно $J_{0}^{-}$) при условии, что результат ненулевой;

б) действием оператора $\left(J_{0}^{-}\right)^{-1}$ (соответственно $\left.\left(J_{0}^{+}\right)^{-1}\right)$, если это действие определено как элемент из $\mathcal{R}$, а результат шага "а" равен нулю.

Подчеркнем, что введение обобшенных потомков - не более чем прием, помогающий путешествовать по экстремальным диаграммам тогда, когда действие собственно $\widehat{s l}(2)$-генераторов обращается в нуль.

КоРАЗмЕРность 3. Исследуем дальнейшие случаи вырождения расслабленных модулей Верма. Теперь мы анализируем возможности одновременного появления трех сингулярных векторов. Начнем со случая, когда имеются два (а потому бесконечно много) расслабленных подмодуля и один заряженный.

ТеОрема 3.8. В расслабленном модуле Верма $\mathcal{R}_{j, \Lambda, k}$ пусть $k=\frac{p}{q}$ рационально и $j=j_{\mathrm{rc}}^{ \pm}(r, s, n, k), \quad \Lambda=\Lambda_{\mathrm{rc}}^{ \pm}(r, s, n, k)$, әде $n \in \mathbb{Z}, \quad r, s \in \mathbb{N}$. Тогда структура модуля $\mathcal{R}_{j_{\mathrm{rc}}^{ \pm}}\left(r, s, n, \frac{p}{q}\right), \Lambda_{\mathrm{rc}}^{ \pm}\left(r, s, n, \frac{p}{q}\right), \frac{p}{q}$ повторяет структуру его подмодуля Верма $\mathcal{C}=\mathcal{M}_{\mathbf{j}^{-}\left(r, s, \frac{p}{q}\right), \frac{p}{q}} \subset \mathcal{R}_{j_{\mathrm{rc}}^{ \pm}\left(r, s, n, \frac{p}{q}\right), \Lambda_{\mathrm{rc}}^{ \pm}\left(r, s, n, \frac{p}{q}\right), \frac{p}{q}}($ ecлu $n<0) u л u \mathcal{C}=\mathfrak{M}_{\mathbf{j}^{+}\left(r, s, \frac{p}{q}\right), \frac{p}{q} ; 1} \subset$ $\mathcal{R}_{j_{\mathrm{rc}}^{ \pm}\left(r, s, n, \frac{p}{q}\right), \Lambda_{\mathrm{rc}}^{ \pm}\left(r, s, n, \frac{p}{q}\right), \frac{p}{q}}($ если $n \geq 0):$ все расслабленные сингулярные векторы $\boldsymbol{в} \mathcal{R}_{j_{\mathrm{rc}}^{ \pm}\left(r, s, n, \frac{p}{q}\right), \Lambda_{\mathrm{rc}}^{ \pm}\left(r, s, n, \frac{p}{q}\right), \frac{p}{q}}$ являются обобщеннылми $J_{0}^{+}$-потомками $($если $n<0)$

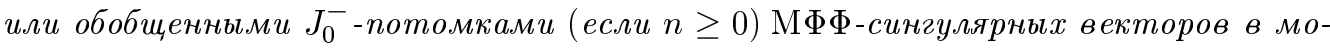
дуле Верма $\mathcal{C}$, вложсенном в $\mathcal{R}_{j_{\mathrm{rc}}^{ \pm}}\left(r, s, n, \frac{p}{q}\right), \Lambda_{\mathrm{rc}}^{ \pm}\left(r, s, n, \frac{p}{q}\right), \frac{p}{q} \cdot$

Остается рассмотреть случай, когда имеются два заряженных подмодуля, каждый из которых допускает М $\Phi$-сингулярный вектор. Разрешая соответствующие условия на $k$, получаем две серии решений:

$$
k+2=\frac{m-n+r}{s} \quad \text { или } \quad k+2=\frac{-m+n+r}{s},
$$

которые рассматриваются в следующей теореме.

ТЕОРема 3.9. Пусть в расслабленном модуле Верма $\mathcal{R}_{j, \Lambda, k}$ параметры выбраны в следующем виде:

I. $j=j_{\mathrm{cc}}(n, m), \quad \Lambda=\Lambda_{\mathrm{cc}}(n, m), \quad k+2=\frac{m-n+r}{s}, \quad r, s \in \mathbb{N}, \quad n \in-\mathbb{N}$. Тогда остаточное $\mathrm{sl}(2)$-представление модуля $\mathcal{R}_{j, \Lambda, k}$ имеет размерность $m-n$. Расслабленный подмодуль Верма порожден любым из состояний

$$
\begin{gathered}
\left(J_{0}^{-}\right)^{-1} \mathcal{M F F}^{+}(r, s+1, k)\left(J_{0}^{-}\right)^{-n}\left|j_{\mathrm{cc}}(n, m), \Lambda_{\mathrm{cc}}(n, m), k\right\rangle_{s l(2)}, \\
\left(J_{0}^{+}\right)^{-1} \mathcal{M} \mathcal{F F}^{-, 1}(r, s+1, k)\left(J_{0}^{+}\right)^{m+1}\left|j_{\mathrm{cc}}(n, m), \Lambda_{\mathrm{cc}}(n, m), k\right\rangle_{s l(2)}
\end{gathered}
$$

с указанным $k$, остаточное $\operatorname{sl}(2)$-представление расслабленного подмодуля Верма имеет размерность $m-n+2 r$.

II. $j=j_{\mathrm{cc}}(n, m), \quad \Lambda=\Lambda_{\mathrm{cc}}(n, m), \quad k+2=\frac{-m+n+r}{s}, \quad r, s, m \in \mathbb{N}, \quad n \in-\mathbb{N}$. Тогда остаточное sl(2)-представление модуля $\mathcal{R}_{j, \Lambda, k}$ имеет размерность $m-n$ В этом случае, кроме того, имеем: 
а) если $2 r-m+n \leq-1$, то расслабленный подмодуль Верма порождается любым из состояний

$$
\begin{aligned}
& \left|s_{1}\right\rangle=\left(J_{0}^{-}\right)^{-1} \mathcal{M F F}^{-}(r, s, k)\left(J_{0}^{-}\right)^{-n}\left|j_{\mathrm{cc}}(n, m), \Lambda_{\mathrm{cc}}(n, m), k\right\rangle_{s l(2)}, \\
& \left|s_{2}\right\rangle=\left(J_{0}^{+}\right)^{-1} \mathcal{M} \mathcal{F F}^{+, 1}(r, s, k)\left(J_{0}^{+}\right)^{m+1}\left|j_{\mathrm{cc}}(n, m), \Lambda_{\mathrm{cc}}(n, m), k\right\rangle_{s l(2)}
\end{aligned}
$$

(әде $\left.k+2=\frac{-m+n+r}{s}\right)$. Остаточное $s l(2)$-представление расслабленного подмодуля Верма имеет размерность $m-n-2 r$;

б) если $2 r-m+n \geq 0$, то каждое из состояний

$$
\begin{aligned}
|\mathrm{mff}\rangle^{-} & =\mathcal{M F F}^{-}(r, s, k)\left(J_{0}^{-}\right)^{-n}\left|j_{\mathrm{cc}}(n, m), \Lambda_{\mathrm{cc}}(n, m), k\right\rangle_{s l(2)}, \\
|\mathrm{mff}\rangle^{+} & =\mathcal{M} \mathcal{F F}^{+, 1}(r, s, k)\left(J_{0}^{+}\right)^{m+1}\left|j_{\mathrm{cc}}(n, m), \Lambda_{\mathrm{cc}}(n, m), k\right\rangle_{s l(2)}
\end{aligned}
$$

порождает в $\mathcal{R}_{j, \Lambda, k}$ подмодули, обозначаемье $\mathcal{M}_{-}$и $\mathcal{M}_{+}$, соответственно, которые не совпадают и объединение которых $\mathcal{M}_{-} \cup \mathcal{M}_{+}$является максимальным подмодулем. Если, кроме того, $2 r-m+n \geq 1$, то $\mathcal{M}_{-}$содерәсит сингулярный вектор $\mathrm{MFF}^{+}\left(2 r-m+n, 1, \frac{-m+n+r}{s}-2\right)$, тогда как $\mathcal{M}_{+}$содержит сингулярный вектор $\mathrm{MFF}^{-, 1}\left(2 r-m+n, 1, \frac{-m+n+r}{s}-2\right)$. При каждом фиксированном $i$ состояния $\left(J_{0}^{+}\right)^{i}|\mathrm{mff}\rangle^{+} u\left(J_{0}^{-}\right)^{2 r-m+n-1-i}|\mathrm{mff}\rangle^{-}, \quad i=0, \ldots, 2 r-m+n-1$, находятся в одной и той же градуировке и линейно независимы. После факторизации по подмодулям, порожденным из векторов $\mathrm{MFF}^{+}\left(2 r-m+n, 1, \frac{-m+n+r}{s}-2\right)$ и $\mathrm{MFF}^{-, 1}\left(2 r-m+n, 1, \frac{-m+n+r}{s}-2\right)$, состояния, остающиеся на верхнем уровне максимального подмодуля $\mathcal{M}_{-} \cup \mathcal{M}_{+}$, дают прямую сум.му двух неприводимых представлений алгебры Ли $\operatorname{sl}(2)$, каждое размерности $2 r-m+n$.

Часть ІІа теоремы 3.9 иллюстрируется диаграммой

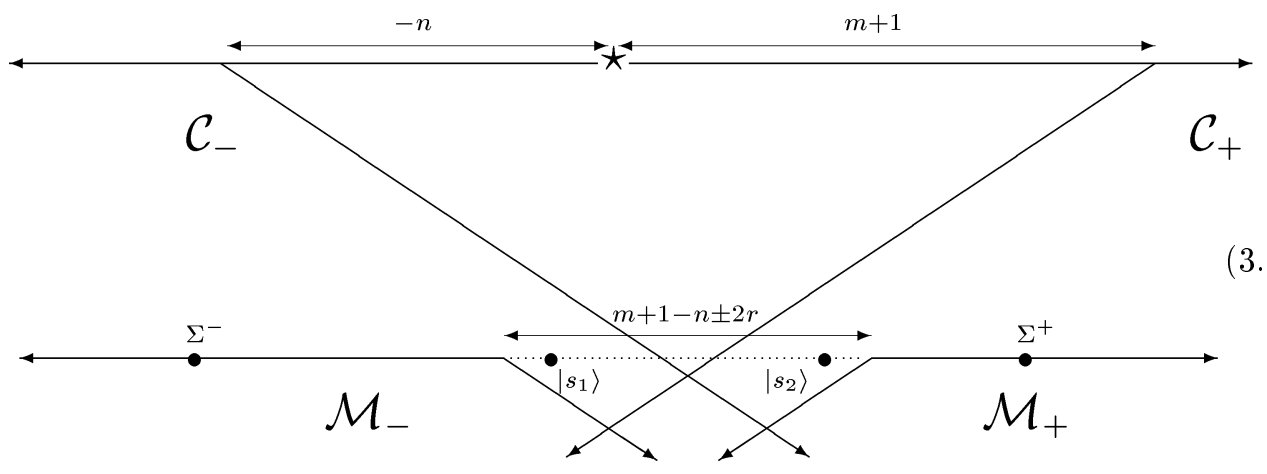

Применительно к случаю ІІб теоремы 3.9 лемма 3.7 означает, что не сушествует обобщенных $J_{0}^{ \pm}$-потомков состояния $|\mathrm{mff}\rangle^{+}$с относительным зарядом меньшим, чем относительный заряд самого вектора $|\mathrm{mff}\rangle^{+}$, поскольку соседнее слева состояние на экстремальной диаграмме оказывается вермовским старшим весом. Аналогично не существует обобщенных $J_{0}^{ \pm}$-потомков вектора $|\mathrm{mff}\rangle^{-}$с относительным зарядом большим, чем относительный заряд вектора $|\mathrm{mff}\rangle^{-}$, и правый сосед этого вектора удовлетворяет вермовским условиям старшего веса, скрученным на $\theta=1$. Этот случай иллюстрируется 


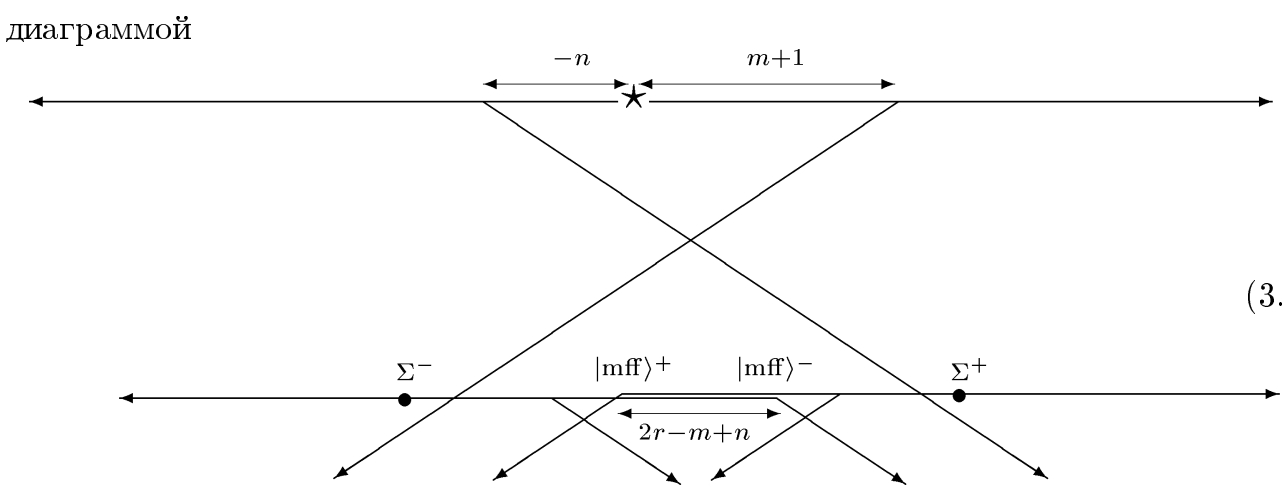

где $|\mathrm{mff}\rangle^{+}$лежит на верхней из двух наложенных друг на друга линий, a $|\mathrm{mff}\rangle^{-}-$на нижней.

3.2. Сингулярные векторы в $N=2$ модулях Верма. Мы опишем сначала сингулярные векторы в топологических модулях Верма. Позднее мы увидим, что эти векторы изоморфны сингулярным векторам в обычных $\widehat{s l}(2)$-модулях Верма, как это ожидалось в [32]. Для построения $N=2$ сингулярных векторов вводятся операторы $g(\mu, \nu)$ и $q(\mu, \nu)$, где $\mu, \nu \in \mathbb{C}$, которые представляют действие двух генераторов $\mathbf{r}_{\alpha_{1}}$ и $\mathbf{r}_{\alpha_{2}}$ " $N=2$ группы Вейля" в случае специальных $\mu$ и $\nu$ (довольно утомительные подробности см. в $[33,40])$. Операторы $g(\mu, \nu)$ и $q(\mu, \nu)$ действуют на плоскости $t=$ const следующим образом:

$$
\begin{gathered}
g(h t+\theta-1, \theta-1):|h, t ; \theta\rangle_{\mathrm{top}} \mapsto\left|\frac{2}{t}-h, t ; h t+\theta-1\right\rangle_{\mathrm{top}}, \\
q(-(h+1) t-\theta+1,-\theta-1):|h, t ; \theta\rangle_{\mathrm{top}} \mapsto\left|\frac{2}{t}-2-h, t ;(h+1) t+\theta-1\right\rangle_{\mathrm{top}} .
\end{gathered}
$$

Действие операторов $g(a, b)$ и $q(a, b)$ на состояния старшего веса можно продолжить на модули Верма, порожденные из таких состояний старшего веса, что позволяет построить сингулярные векторы в $N=2$ модулях Верма.

Алгебра продолженных операторов. Операторы $g(a, b)$ и $q(a, b)$ можно рассматривать как продолженные произведения мод операторов $\mathcal{G}_{a} \mathcal{G}_{a+1} \ldots \mathcal{G}_{a+N}$ и $\mathcal{Q}_{a} \mathcal{Q}_{a+1} \ldots \mathcal{Q}_{a+N}$ на случай нецелого (комплексного) числа множителей. Когда же длина $b-a+1$ оператора $g(a, b)$ или $q(a, b)$ становится целым числом, оператор по определению преврашается в произведение мод:

$$
g(a, b)=\prod_{i=0}^{L-1} \mathcal{G}_{a+i}, \quad q(a, b)=\prod_{i=0}^{L-1} \mathcal{Q}_{a+i}, \quad \text { если } \quad L \equiv b-a+1=0,1,2, \ldots
$$

Идея "плотного заполнения" формализуется в следуюших правилах:

$$
\begin{aligned}
g(a, b-1) g(b, \theta-1)|\theta\rangle_{g} & =g(a, \theta-1)|\theta\rangle_{g}, \quad a, b, \theta \in \mathbb{C}, \\
q(a, b-1) q(b,-\theta-1)|\theta\rangle_{q} & =q(a,-\theta-1)|\theta\rangle_{q}, \quad
\end{aligned}
$$

где $|\theta\rangle_{g}$ - любое состояние, удовлетворяющее условиям $\mathcal{G}_{\theta+n}|\theta\rangle_{g}=0$ при $n \in \mathbb{N}_{0}$, тогда как $|\theta\rangle_{q}$ удовлетворяет $\mathcal{Q}_{-\theta+n}|\theta\rangle_{q}=0$ при $n \in \mathbb{N}_{0}$. Подобным же образом имеем 
условия обрашения в нуль, отражающие фермионный характер $N=2$ генераторов $\mathcal{Q}$ и $\mathcal{G}$ :

$\mathcal{G}_{a} g(b, c)=0, \quad \mathcal{Q}_{a} q(b, c)=0, \quad a-b \in \mathbb{N}_{0} \quad$ и $\quad(a-c \notin \mathbb{N} \quad$ или $\quad b-c-1 \in \mathbb{N})$,

а также “левосторонние" условия аннигиляции

$g(a, b) \mathcal{G}_{c}=0, \quad q(a, b) \mathcal{Q}_{c}=0, \quad b-c \in \mathbb{N}_{0} \quad$ и $\quad(a-c \notin \mathbb{N} \quad$ или $\quad a-b-1 \in \mathbb{N})$

Для алгебраических манипуляций требуются также правила коммутации продолженных операторов с бозонными операторами $\mathcal{L}_{\geq 1}$ и $\mathcal{H}_{\geq 1}$ :

$$
\begin{aligned}
{\left[\mathcal{K}_{p}, g(a, b)\right] } & =\sum_{l=0}^{d(p, a, b)} g(a, b-l-1)\left[\mathcal{K}_{p}, \mathcal{G}_{b-l}\right] \mathcal{G}_{b-l+1} \ldots \mathcal{G}_{b}, \quad p \in \mathbb{N}, \\
{\left[\mathcal{K}_{p}, q(a, b)\right] } & =\sum_{l=0}^{d(p, a, b)} q(a, b-l-1)\left[\mathcal{K}_{p}, \mathcal{Q}_{b-l}\right] \mathcal{Q}_{b-l+1} \ldots \mathcal{Q}_{b},
\end{aligned}
$$

где

$$
d(n, a, b)= \begin{cases}b-a, & n-b+a \in \mathbb{N}_{0} \text { и } b-a+1 \in \mathbb{N}_{0}, \\ n-1 & \text { в остальных случаях, }\end{cases}
$$

и $\mathcal{K}=\mathcal{L}$ или $\mathcal{H}$. Операторы $g$ и $q$ изменяют также собственные значения $\mathcal{L}_{0}$ и $\mathcal{H}_{0}$, что выражается следующими коммутационными соотношениями:

$$
\begin{array}{ll}
{\left[\mathcal{L}_{0}, g(a, b)\right]=-\frac{1}{2}(a+b)(b-a+1) g(a, b),} & {\left[\mathcal{H}_{0}, g(a, b)\right]=(b-a+1) g(a, b),} \\
{\left[\mathcal{L}_{0}, q(a, b)\right]=-\frac{1}{2}(a+b)(b-a+1) q(a, b),} & {\left[\mathcal{H}_{0}, q(a, b)\right]=(-b+a-1) q(a, b) .}
\end{array}
$$

Под действием фермионных генераторов имеем

$$
\begin{gathered}
\mathcal{Q}_{-\theta+n} g(\theta,-1)|h, \ell, t\rangle=0, \quad \theta \in \mathbb{C}, \quad n \in \mathbb{N}, \\
\mathcal{Q}_{-\theta} g(\theta,-1)|h, \ell, t\rangle=2\left(\ell+\theta h-\frac{1}{t}\left(\theta^{2}+\theta\right)\right) g(\theta+1,-1)|h, \ell, t\rangle,
\end{gathered}
$$

а для $q$-операторов -

$$
\begin{gathered}
\mathcal{G}_{\theta+n} q(-\theta, 0)|h, \ell, t\rangle=0, \quad n \in \mathbb{N}, \\
\mathcal{G}_{\theta} q(-\theta, 0)|h, \ell, t\rangle=2\left(\ell+\theta h-\frac{1}{t}\left(\theta^{2}+\theta\right)\right) q(-\theta+1,0)|h, \ell, t\rangle .
\end{gathered}
$$

Наконец, формулы для коммутации отрицательных мод операторов $\mathcal{H}$ и $\mathcal{L}$ с продолженными операторами $q(a, b)$ и $g(a, b)$ имеют вид

$$
\begin{aligned}
& {\left[g(a, b), \mathcal{K}_{p}\right]=\sum_{l=0}^{d(-p, a, b)} \mathcal{G}_{a} \ldots \mathcal{G}_{a+l-1}\left[\mathcal{G}_{a+l}, \mathcal{K}_{p}\right] g(a+l+1, b),} \\
& {\left[q(a, b), \mathcal{K}_{p}\right]=\sum_{l=0}^{d(-p, a, b)} \mathcal{Q}_{a} \ldots \mathcal{Q}_{a+l-1}\left[\mathcal{Q}_{a+l}, \mathcal{K}_{p}\right] q(a+l+1, b),}
\end{aligned}
$$

где $d(p, a, b)$ дается формулой $(3.36)$ и, как и раньше, $\mathcal{K}=\mathcal{H}$ или $\mathcal{L}$.

Соотношения (3.31)-(3.40) являются $N=2$ аналогом равенств (3.2).

Топологические сингулярные векторы. Теперь $N=2$ сингулярные векторы получаются в весьма простой, мономиальной, форме. 
TEOPEMA 3.10 .

I ([10]). Топологический сингулярный вектор существует в топологическом модуле Верма $\mathcal{V}_{h, t}$, если и только если $h=\mathbf{h}^{+}(r, s, t)$ или $h=\mathbf{h}^{-}(r, s, t)$, әде

$$
\mathbf{h}^{+}(r, s, t)=-\frac{r-1}{t}+s-1, \quad \mathbf{h}^{-}(r, s, t)=\frac{r+1}{t}-s, \quad r, s \in \mathbb{N} .
$$

II. Все сингулярные векторы в топологическом модуле Верма $\mathcal{V}_{\mathbf{h}^{ \pm}(r, s, t), t}$ даются следующей конструкиией:

$$
\begin{aligned}
|E(r, s, t)\rangle^{+}=g( & -r,(s-1) t-1) q(-(s-1) t, r-1-t) \ldots g((s-2) t-r, t-1) \times \\
& \times q(-t, r-1-t(s-1)) g((s-1) t-r,-1)\left|\mathbf{h}^{+}(r, s, t), t\right\rangle_{\mathrm{top}} \\
|E(r, s, t)\rangle^{-}= & (-r,(s-1) t-1) g(-(s-1) t, r-t-1) \ldots q((s-2) t-r, t-1) \times \\
& \times g(-t, r-1-(s-1) t) q((s-1) t-r,-1)\left|\mathbf{h}^{-}(r, s, t), t\right\rangle_{\mathrm{top}}
\end{aligned}
$$

Сингулярные векторы (3.42), (3.43) удовлетворяют условиям старшего веса (2.14), в которых $\theta=\mp r$, соответственно.

Сингулярные векторы в массивных модулях Верма. Те же операторы $g(a, b)$ и $q(a, b)$ позволяют построить сингулярные векторы в массивных $N=2$ модулях Верма [33]. Начнем, однако, с простейшего случая, непосредственно следуюшего из анализа экстремальных диаграмм. Итерируя формулы (2.22), мы получаем следуюшую теоремy.

Tеорема 3.11. Массивный модуль Верма $U_{h, \ell, t}$ содержит скрученный топологический подмодуль, если и только если $\ell=\mathrm{l}_{\mathrm{ch}}(r, h, t)$, әде

$$
\mathbf{l}_{\mathrm{ch}}(r, h, t)=r\left(h+\frac{r-1}{t}\right), \quad r \in \mathbb{Z} .
$$

Соответствующие сингулярные векторы имеют вид

$$
|E(r, h, t)\rangle_{\mathrm{ch}}= \begin{cases}\mathcal{Q}_{r} \ldots \mathcal{Q}_{0}\left|h, \mathbf{1}_{\mathrm{ch}}(r, h, t), t\right\rangle, & r \leq-1 \\ \mathcal{G}_{-r} \ldots \mathcal{G}_{-1}\left|h, \mathbf{l}_{\mathrm{ch}}(r, h, t), t\right\rangle, & r \geq 0\end{cases}
$$

Они удовлетворяют скрученным топологическим условиям старшего веса (2.14), где $\theta=-r$.

(Эти сингулярные векторы - так называемая "заряженная" серия из [10].)

Оставшиеся сингулярные векторы в массивных модулях Верма строятся следуюшим образом.

TEOpema 3.12 .

I ([10]). Массивный сингулярный вектор существует в $U_{h, \ell, t}$, если $\ell=\ell(r, s, h, t)$ при $r, s \in \mathbb{N}$, где $(\partial л я \mathbf{c} \neq 3)$

$$
\ell(r, s, h, t)=\frac{1}{2} h-\frac{1}{2} r s-\frac{1}{4 t}+\frac{r^{2}}{4 t}-\frac{1}{4} h^{2} t+\frac{1}{4} s^{2} t, \quad r, s \in \mathbb{N} .
$$


II. Массивные сингулярные векторы имеют вид

$$
\begin{aligned}
&|S(r, s, h, t)\rangle^{-}=g(\left.-r s, r+\theta^{-}(r, s, h, t)-1\right) \times \\
& \times \mathcal{E}^{-, \theta^{-}(r, s, h, t)}(r, s) g\left(\theta^{-}(r, s, h, t),-1\right)|h, \ell(r, s, h, t), t\rangle, \\
&|S(r, s, h, t)\rangle^{+}=q\left(1-r s, r-\theta^{+}(r, s, h, t)-1\right) \times \\
& \quad \times \mathcal{E}^{+, \theta^{+}(r, s, h, t)}(r, s) q\left(-\theta^{+}(r, s, h, t), 0\right)|h, \ell(r, s, h, t), t\rangle,
\end{aligned}
$$

где

$$
\theta^{-}(r, s, h, t)=\frac{t}{2}\left(h-\mathbf{h}^{-}(r, s, t)\right), \quad \theta^{+}(r, s, h, t)=\frac{t}{2}\left(h-1-\mathbf{h}^{+}(r, s, t)\right)
$$

$u \mathcal{E}^{ \pm, \theta}(r, s, t)$ обозначает отображсение спектрального потока $(2.16)$, примененное $\kappa$ оператору топологического сингулярного вектора $\mathcal{E}^{ \pm}(r, s, t)$.

Два выражсния (3.47) в случае общего положсения дают состояния, лежащие на одной и той жее экстремальной диаграмме, и каждое из них порожсдет весь массивный подмодуль.

Все определенные выше $N=2$ сингулярные векторы вычисляются путем многократного применения формул (3.31)-(3.40) как элементы соответствующих модулей Верма, т.е. в виде полиномов по соответствуюшим операторам рождения.

Аналогично тому, как это было сделано для алгебры $\widehat{s l}(2)$, можно теперь проанализировать все случаи одновременного появления сингулярных векторов. Мы не будем здесь этого делать в полной мере (см. [36]) и приведем лиш наиболее интересный случай в коразмерности 3 , когда одновременно сушествуют два заряженных и один массивный сингулярные векторы. В нижеследуюших конструкциях участвуют операторы $g(a+N, a-1)$ и $q(a+N, a-1)$ целой отрицательной длины $-N$. Правила обращения с такими операторами, действующими на состояние старшего веса, следуют из приведенных выше формул (3.31)-(3.40), откуда, в частности, имеем

$$
g(\theta+1, \theta-1)|h, \ell, t ; \theta\rangle=\frac{1}{2\left(\ell+h-\frac{2}{t}\right)} \mathcal{Q}_{-\theta}|h, \ell, t ; \theta\rangle .
$$

Определим еще

$$
\mathbf{h}_{\mathrm{cc}}(n, m, t)=\frac{1}{t}(1-m-n), \quad \mathbf{l}_{\mathrm{cc}}(n, m, t)=-\frac{m n}{t} .
$$

ТеОрема 3.13. Пусть параметры массивного модуля Верма $\mathcal{U}_{h, \ell, t}$ выбраны в виде:

I. $h=\mathbf{h}_{\mathrm{cc}}(n, m, t), \quad \ell=\mathbf{l}_{\mathrm{cc}}(n, m, t), \quad t=\frac{-m+n+r}{s}, \quad r, s, n \in \mathbb{N}, m \in-\mathbb{N}_{0}$. Тогдa массивный подмодуль Верма порождается любъм из состояний

$$
\begin{gathered}
g(-r-n+1,-r-n-1) \mathcal{E}^{+,-n}(r, s+1, t) g(-n,-1)\left|\mathbf{h}_{\mathrm{cc}}(n, m, t), \mathbf{l}_{\mathrm{cc}}(n, m, t), t\right\rangle, \\
q(-r+m+1,-r+m-1) \mathcal{E}^{-,-m}(r, s+1, t) q(m, 0)\left|\mathbf{h}_{\mathrm{cc}}(n, m, t), \mathbf{l}_{\mathrm{cc}}(n, m, t), t\right\rangle
\end{gathered}
$$

с указанным значением $t$.

II. $h=\mathbf{h}_{\mathrm{cc}}(n, m, t), \quad \ell=\mathbf{1}_{\mathrm{cc}}(n, m, t), \quad t=\frac{m-n+r}{s}, \quad r, s, n \in \mathbb{N}, \quad m \in-\mathbb{N}_{0}$, тогда:

а) если $2 r+m-n \leq-1$, то массивный подмодуль Верма порождается любьм из состояний

$$
\begin{gathered}
g(r-n+1, r-n-1) \mathcal{E}^{-,-n}(r, s, t) g(-n,-1)\left|\mathbf{h}_{\mathrm{cc}}(n, m, t), \mathbf{l}_{\mathrm{cc}}(n, m, t), t\right\rangle, \\
q(r+m+1, r+m-1) \mathcal{E}^{+,-m}(r, s, k) q(m, 0)\left|\mathbf{h}_{\mathrm{cc}}(n, m, t), \mathbf{l}_{\mathrm{cc}}(n, m, t), t\right\rangle
\end{gathered}
$$


где $t=\frac{m-n+r}{s}$. Расстояние вдоль әкстремальной диаграммы между заряженными сингулярными векторами в массивном подмодуле Верма равно $-m+n+1-2 r$;

б) если $2 r+m-n \geq 0$, то каждое из состояний

$$
\begin{aligned}
\left|e_{-}\right\rangle & =\mathcal{E}^{-,-n}(r, s, t) g(-n,-1)\left|\mathbf{h}_{\mathrm{cc}}(n, m, t), \mathbf{l}_{\mathrm{cc}}(n, m, t), t\right\rangle \\
\left|e_{+}\right\rangle & =\mathcal{E}^{+,-m}(r, s, t) q(m, 0)\left|\mathbf{h}_{\mathrm{cc}}(n, m, t), \mathbf{l}_{\mathrm{cc}}(n, m, t), t\right\rangle
\end{aligned}
$$

порохдает топологический подмодуль в $\mathcal{U}_{h, \ell, t}$, обозначаемый $\mathcal{V}_{-} u \mathcal{V}_{+}$, соответственно. Эти два подмодуля не совпадают, и их обвединение $\mathcal{V}_{-} \cup \mathcal{V}_{+}$представляет собой максимальный подмодуль. Если, кроме того, $2 r+m-n \geq 1$, то $\mathcal{V}_{-}$ содержит сингулярный вектор

$$
\mathcal{E}^{+, r-n}\left(2 r+m-n, 1, \frac{m-n+r}{s}-2\right)\left|e_{-}\right\rangle,
$$

а $\mathcal{V}_{+}-$сингулярный вектор

$$
\mathcal{E}^{-,-m-r}\left(2 r+m-n, 1, \frac{m-n+r}{s}-2\right)\left|e_{+}\right\rangle .
$$

Для каждого $i=0, \ldots, 2 r+m-n-1$ состояние $\mathcal{Q}_{r+m-i} \ldots \mathcal{Q}_{r+m-1}\left|e_{+}\right\rangle$и состояние $\mathcal{G}_{-m-r+i+1} \ldots \mathcal{G}_{r-n-1}\left|e_{-}\right\rangle$лежат в одной и той же градуировке и линейно независимы.

Случай ІІб теоремы 3.13 иллюстрируется следующим образом:

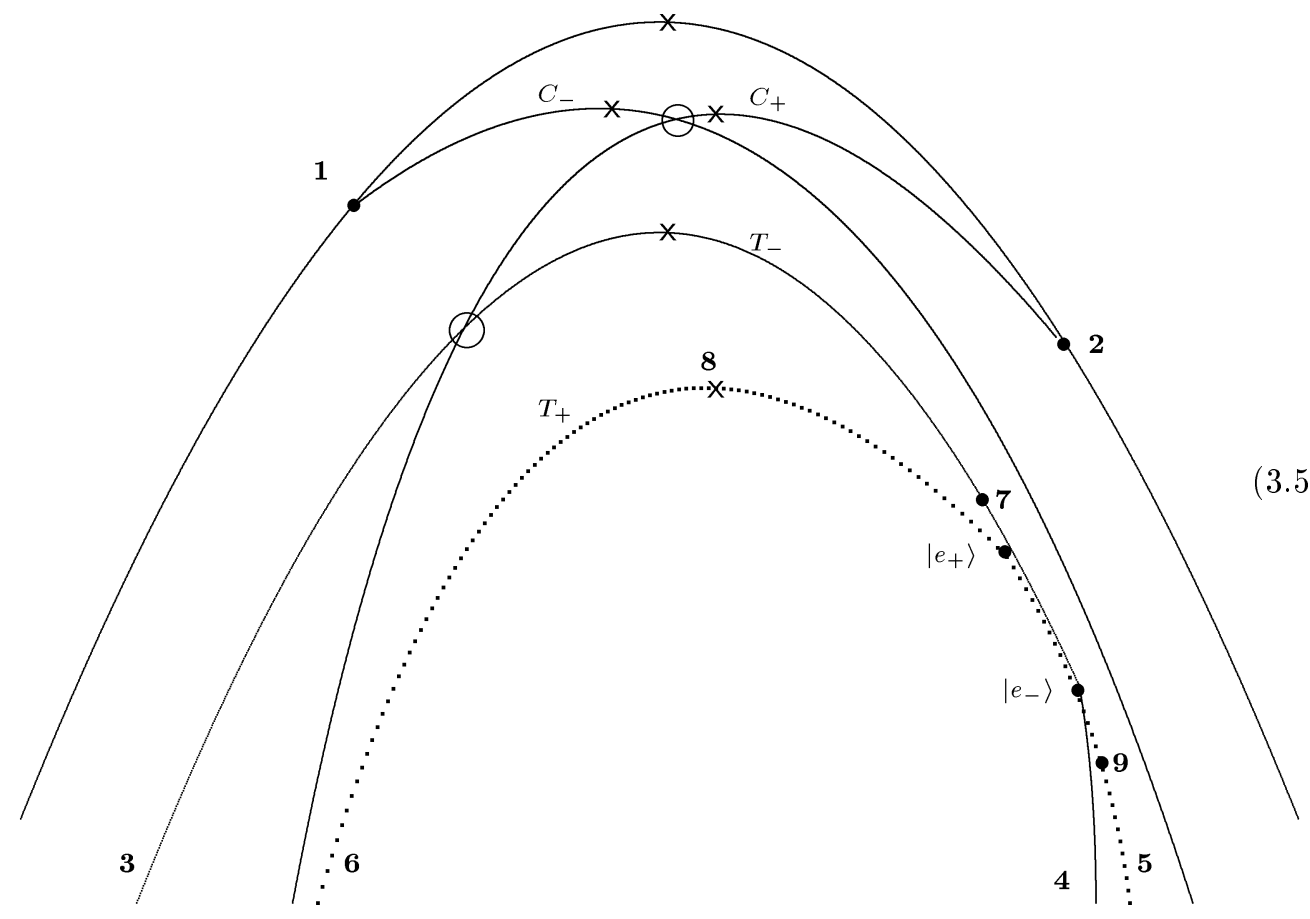


Здесь $\mathbf{1}$ и $\mathbf{2}$ - заряженные сингулярные векторы в массивном модуле Верма $\mathcal{U}$. Соответствующие топологические подмодули в $\mathcal{U}$ имеют экстремальные диаграммы, обозначенные $C_{-}$и $C_{+}$, соответственно. Рассматриваемый вырожденный случай означает, что каждый из топологических подмодулей имеет по топологическому подмодулю, чьи экстремальные диаграммы - это $\mathbf{3}-\left|e_{-}\right\rangle-\mathbf{4}$ (с изломом в точке $\left.\left|e_{-}\right\rangle\right)$и $\mathbf{5}-\left|e_{+}\right\rangle-\mathbf{6}$ (с изломом в $\left.\left|e_{+}\right\rangle\right)$. Состояния из двух подмодулей, расположенные между $\left|e_{-}\right\rangle$и $\left|e_{+}\right\rangle$, находятся в одной и той же градуировке. Состояние $\mathbf{7}$ является еше одним топологическим состоянием старшего веса (в частности, имеем $\mathcal{Q}_{r+m} \mathbf{7}=0$ ) и аналогично для состояния 9.

Наконец, случай ІІа теоремы 3.13 описывается следующим образом:

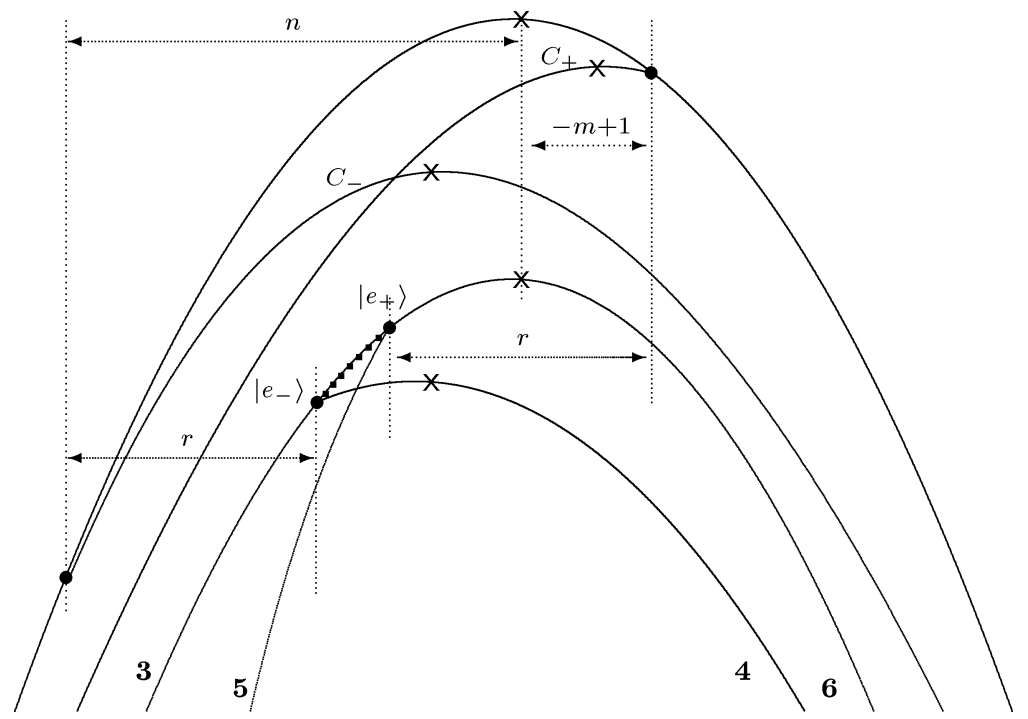

Здесь 3-|$\left.e_{-}\right\rangle-\mathbf{4}$ - экстремальная диаграмма топологического подмодуля $\mathcal{V}_{-}$, а $\mathbf{5}-\left|e_{+}\right\rangle-\mathbf{6}$ - экстремальная диаграмма топологического подмодуля $\mathcal{V}_{+}$. Имеется $-m+n-2 r$ состояний между $\left|e_{-}\right\rangle$и $\left|e_{+}\right\rangle$, которые удовлетворяют скрученным массивным условиям старшего веса, но не лежат ни в одном из подмодулей $\mathcal{V}_{-}$и $\mathcal{V}_{+}$, ни в действительности в каком-либо из подмодулей $C_{-}$или $C_{+}$.

3.3. Сингулярные векторы в $\widehat{s l}(2 \mid 1)$-модулях Верма. Как и в рассмотренных выше случаях, имеются $\widehat{s l}(2 \mid 1)$-сингулярные векторы, структура которых немедленно следует из анализа экстремальных диаграмм, и мы оставим за такими векторами название "заряженные"; остальные $\widehat{s l}(2 \mid 1)$-сингулярные векторы будут также по аналогии (которую мы сделаем ниже не только формальной) с $N=2$ алгеброй назьваться массивными.

“Заряженные" $\widehat{s l}(2 \mid 1)$ 1-сингулярные векторы. Как видно из диаграммы (2.31), сингулярные векторы появляются при следующих значениях параметров:

$\mathbf{p}_{2}(r, j, \kappa)=-j+\frac{1}{2} \kappa-r(\kappa+1), \quad r \in \mathbb{Z}, \quad$ или $\quad \mathbf{p}_{1}(r, j, \kappa)=j+\frac{1}{2} \kappa-r(\kappa+1), \quad r \in \mathbb{Z}$. 
Первые из них, которые мы будем называть заряженными-II сингулярными векторами, имеют вид (см. конструкцию (2.29) для экстремальных состояний)

$$
\begin{aligned}
& |E(r, j, \kappa)\rangle_{\mathrm{ch}}^{(2)}= \\
& \quad=\left\{\begin{array}{lll}
E_{r-\frac{1}{2}}^{2} \ldots E_{-\frac{3}{2}}^{2} \cdot F_{r+\frac{1}{2}}^{1} \ldots F_{-\frac{1}{2}}^{1}\left|\mathbf{p}_{2}(r, j, \kappa), j, \kappa\right\rangle, & r \leq-1, \\
E_{-r+\frac{1}{2}}^{1} \ldots E_{-\frac{1}{2}}^{1} \cdot F_{-r+\frac{1}{2}}^{2} F_{-r+\frac{3}{2}}^{2} \ldots F_{\frac{1}{2}}^{2}\left|\mathbf{p}_{2}(r, j, \kappa), j, \kappa\right\rangle, & r \geq 0,
\end{array}\right.
\end{aligned}
$$

ЛЕмма 3.14. Заряэсенные-ІІ сингулярные векторы $|E(r, j, \kappa)\rangle_{\mathrm{ch}}^{(2)}$ удовлетворяют случаю $\theta=r$ условий старшего веса

$$
\begin{gathered}
E_{\geq-\theta+\frac{1}{2}}^{1} \approx 0, \quad E_{\geq \theta-\frac{1}{2}}^{2} \approx 0, \quad E_{\geq 0}^{12} \approx 0, \quad H_{\geq 1}^{+} \approx 0, \\
F_{\geq \theta+\frac{1}{2}}^{1} \approx 0, \quad F_{\geq-\theta+\frac{1}{2}}^{2} \approx 0, \quad F_{\geq 1}^{12} \approx 0, \quad H_{\geq 1}^{-} \approx 0, \\
\left(\theta-\frac{1}{2}\right) \kappa+H_{0}^{-}+H_{0}^{+} \approx 0 .
\end{gathered}
$$

Такие условия старшего веса - более сильные, чем (2.28) - будут называться топологическими (и еще скрученными, коль скоро $\theta \neq 0$ ). В алгебре $\widehat{s l}(2 \mid 1)$ существуют два типа топологических условий старшего веса и соответственно два типа топологических сингулярных векторов.

Заряженные-І сингулярные векторы строятся как

$$
|E(r, j, \kappa)\rangle_{\mathrm{ch}}^{(1)}=\left\{\begin{array}{ll}
E_{r-\frac{1}{2}}^{2} \ldots E_{-\frac{3}{2}}^{2} \cdot F_{r-\frac{1}{2}}^{1} F_{r+\frac{1}{2}}^{1} \ldots F_{-\frac{1}{2}}^{1}\left|\mathbf{p}_{1}(r, j, \kappa), j, \kappa\right\rangle, \quad r \leq 0, \\
E_{-r+\frac{1}{2}}^{1} \ldots E_{-\frac{1}{2}}^{1} \cdot F_{-r+\frac{3}{2}}^{2} \ldots F_{\frac{1}{2}}^{2}\left|\mathbf{p}_{1}(r, j, \kappa), j, \kappa\right\rangle, & r \geq 1,
\end{array} \quad r \in \mathbb{Z} .\right.
$$

ЛЕмМа 3.15. Заряжсенные-I сингулярные векторы (3.59) удовлетворяют условиям стариего веса

$$
\begin{array}{ccc}
E_{\geq-\theta+\frac{1}{2}}^{1} \approx 0, & E_{\geq \theta-\frac{1}{2}}^{2} \approx 0, \quad E_{\geq 0}^{12} \approx 0, & H_{\geq 1}^{+} \approx 0 \\
F_{\geq \theta-\frac{1}{2}}^{1} \approx 0, \quad F_{\geq-\theta+\frac{3}{2}}^{2} \approx 0, & F_{\geq 1}^{12} \approx 0, & H_{\geq 1}^{-} \approx 0, \\
\left(\theta-\frac{1}{2}\right) \kappa-H_{0}^{-}+H_{0}^{+} \approx 0 . &
\end{array}
$$

Эти условия будут называться (скрученными) топологическими-I условиями старшего веса.

МФФ-конструкция для “массивных" $\widehat{s l}(2 \mid 1)$-сингулярных векторов. Теперь мы перейдем к "продолженной" конструкции для $\widehat{s l}(2 \mid 1)$-сингулярных векторов. Их положения (как, впрочем, и положения заряженных) известны, конечно, из анализа детерминанта Каца-Каждана и могут быть найдены в работе [41] (см. также цитированную там литературу). Ниже мы построим конструкцию для этих сингулярных векторов при произвольных (комплексных) значениях уровня $\kappa$. На наиболее фундаментальном уровне такая обобшенная М $\Phi \Phi$-конструкция допускает переформулировку в терминах одних только “продолженных" фермионных генераторов алгебры $\widehat{s l}(2 \mid 1)$, что роднит ее с конструкцией для $N=2$ сингулярных векторов. 
Случай общего положения. Определим ${ }^{5)}$

$$
\mathbf{j}^{+}(r, s, \kappa)=\frac{r}{2}-\frac{s-1}{2}(\kappa+1), \quad \mathbf{j}^{-}(r, s, \kappa)=-\frac{r}{2}+\frac{s}{2}(\kappa+1), \quad r, s \in \mathbb{N},
$$

тогда имеем теорему

Теорема 3.16. Для комплексных значений $U(1)$ заряда р и уровня $\kappa$ в случае общего положения мономиальные выражения

$$
\begin{gathered}
|\operatorname{MFF}(r, s, p, \kappa)\rangle^{+}=E_{\frac{1}{2}}^{1}\left(F_{0}^{12}\right)^{r+(s-1)(\kappa+1)} F_{-\frac{1}{2}}^{1} \cdot\left(E_{-1}^{12}\right)^{r+(s-2)(\kappa+1)} \times \cdots \\
\cdots \times E_{\frac{1}{2}}^{1}\left(F_{0}^{12}\right)^{r-(s-1)(\kappa+1)} F_{-\frac{1}{2}}^{1}\left|p, \mathbf{j}^{+}(r, s, \kappa), \kappa\right\rangle \\
|\operatorname{MFF}(r, s, p, \kappa)\rangle^{-}=\left(E_{-1}^{12}\right)^{r+(s-1)(\kappa+1)} \cdot E_{\frac{1}{2}}^{1}\left(F_{0}^{12}\right)^{r+(s-2)(\kappa+1)} F_{-\frac{1}{2}}^{1} \times \cdots \\
\cdots \times\left(E_{-1}^{12}\right)^{r-(s-1)(\kappa+1)}\left|p, \mathbf{j}^{-}(r, s, \kappa), \kappa\right\rangle
\end{gathered}
$$

(әде $r, s \in \mathbb{N}$ ) определяют сингулярные векторы в модулях Верма, построенных на векторах стариего веса $\left|p, \frac{r}{2}-\frac{s-1}{2}(\kappa+1), \kappa\right\rangle$ u $\left|p, \frac{-r}{2}+\frac{s}{2}(\kappa+1), \kappa\right\rangle$, соответственно.

Мы будем называть эти сингулярные векторы массивными.

Для придания смысла этой алгебраически продолженной конструкции используются следуюшие коммутационные соотношения (являюшиеся, очевидно, обобшением формул (3.2)):

$$
\begin{aligned}
\left(F_{0}^{12}\right)^{n} E_{m}^{1} & =\left(-n F_{m}^{2}+E_{m}^{1} F_{0}^{12}\right)\left(F_{0}^{12}\right)^{n-1}, \\
\left(E_{-1}^{12}\right)^{n} F_{m}^{1} & =\left(n E_{m-1}^{2}+F_{m}^{1} E_{-1}^{12}\right)\left(E_{-1}^{12}\right)^{n-1}, \\
\left(F_{0}^{12}\right)^{n} E_{m}^{2} & =\left(n F_{m}^{1}+E_{m}^{2} F_{0}^{12}\right)\left(F_{0}^{12}\right)^{n-1}, \\
\left(E_{-1}^{12}\right)^{n} F_{m}^{2} & =\left(-n E_{m-1}^{1}+F_{m}^{2} E_{-1}^{12}\right)\left(E_{-1}^{12}\right)^{n-1}, \\
\left(F_{0}^{12}\right)^{n} H_{m}^{-} & =\left(n F_{m}^{12}+H_{m}^{-} F_{0}^{12}\right)\left(F_{0}^{12}\right)^{n-1}, \\
\left(E_{-1}^{12}\right)^{n} H_{m}^{-} & =\left(-n E_{m-1}^{12}+H_{m}^{-} E_{-1}^{12}\right)\left(E_{-1}^{12}\right)^{n-1}, \\
\left(F_{0}^{12}\right)^{n} E_{m}^{12}= & \left(-n(n-1) F_{m}^{12}-2 n H_{m}^{-} F_{0}^{12}+E_{m}^{12} F_{0}^{12} F_{0}^{12}\right)\left(F_{0}^{12}\right)^{n-2}, \\
\left(E_{-1}^{12}\right)^{n} F_{m}^{12}= & \left(-n(n-1) E_{m-2}^{12}-\kappa n \delta_{m-1,0} E_{-1}^{12}+\right. \\
& \left.+2 n H_{m-1}^{-} E_{-1}^{12}+F_{m}^{12} E_{-1}^{12} E_{-1}^{12}\right)\left(E_{-1}^{12}\right)^{n-2} .
\end{aligned}
$$

Последовательное использование формул (3.63) в выражениях для $|\operatorname{MFF}(r, s, p, \kappa)\rangle^{ \pm}$ приводит в конце конщов к выражениям, явным образом представляющим собой элементы модуля Верма.

\footnotetext{
5)Что является злоупотреблением обозначениями; мы просим читателя забыть на время об определениях (3.3), а также об обозначении MFF для $\widehat{s l}(2)$-сингулярных векторов.
} 
Обешанное представление тех же сингулярных векторов через одни только "продолженные” фермионные генераторы имеет вид

$$
\begin{aligned}
& |\operatorname{MFF}(r, s, p, \kappa)\rangle^{+}= \\
& =E_{\frac{1}{2}}^{1} f^{2}\left(\frac{3}{2}, r+\frac{1}{2}+(s-1)(\kappa+1)\right) f^{1}\left(-r-\frac{1}{2}-(s-1)(\kappa+1), \frac{1}{2}\right) \times \\
& \quad \times e^{2}\left(\frac{1}{2},-r-\frac{1}{2}+(s-2)(\kappa+1)\right) e^{1}\left(r+\frac{1}{2}-(s-2)(\kappa+1), \frac{1}{2}\right) \times \\
& \quad \times f^{2}\left(\frac{3}{2}, r+\frac{1}{2}-(s-1)(\kappa+1)\right) f^{1}\left((s-1)(\kappa+1)-r-\frac{1}{2},-\frac{1}{2}\right)\left|p, \mathbf{j}^{+}(r, s, \kappa), \kappa\right\rangle
\end{aligned}
$$

и аналогично для $\mathrm{MFF}^{-}$. Здесь, например, $f^{1}(a, b)^{\text {“ }}=" \prod_{a}^{b} F_{\alpha}^{1}$, чему следует придать смысл аналогично тому, как это делается для "продолженных произведений" фермионных генераторов $N=2$ алгебры.

Сингулярные векторы в "рамоновском" (или в любом другом) секторе, которые мы используем в следуюшем разделе, получаются из вьписанных вьше сингулярных векторов применением отображения спектрального потока, что можно сделать непосредственно в мономиальной форме (3.62), (3.57) и (3.59).

Конструкцию состояний $\mathrm{MFF}^{ \pm}$можно формально определить и при $r=0$, в этом случае они оказываются пропорциональны самим состояниям старшего веса (или обращаются в нуль), например

$$
\begin{aligned}
& |\operatorname{MFF}(0, s, p, \kappa)\rangle^{+}=a(s, p, \kappa)\left|p, \mathbf{j}^{+}(0, s, \kappa), \kappa\right\rangle, \\
& a(s, p, \kappa)= \begin{cases}\left(p-\frac{\kappa}{2}\right) \prod_{i=1}^{(s-1) / 2}\left(p-\frac{\kappa}{2}+i(\kappa+1)\right)\left(p-\frac{\kappa}{2}-i(\kappa+1)\right), & s \text { четное, } \\
\prod_{i=1}^{s / 2}\left(p-\frac{\kappa}{2}+\left(i-\frac{1}{2}\right)(\kappa+1)\right)\left(p-\frac{\kappa}{2}-\left(i-\frac{1}{2}\right)(\kappa+1)\right), & s \text { нечетное, }\end{cases}
\end{aligned}
$$

и аналогично для $|\operatorname{MFF}(0, s, p, \kappa)\rangle^{-}$.

“Исключительные" точки. Мы не собираемся описывать здесь все возможные вырожденные случаи еше и для $\widehat{s l}(2 \mid 1)$-алгебры. Вместо этого мы обращаем внимание на особенность $\widehat{s l}(2 \mid 1)$-случая, отсутствующую в стандартной МФФ-конструкции для $A_{n}^{(1)}$, а именно на тот факт, что $\widehat{s l}(2 \mid 1)$-формулы МФФ-типа могут давать нулевой результат в определенных точках в пространстве $p, j, \kappa$ старших весов. Возможность такого обрашения в нуль связана тем, что возвращение от модифицированных условий старшего веса (2.32) к стандартным может оказаться невозможным.

Прежде всего заметим, что массивные сингулярные векторы альтернативным образом даются алгебраической конструкцией, отличающейся от (3.62) заменой $E^{1}$ и $F^{1}$ соответственно на $E^{2}$ и $F^{2}$, например

$$
\begin{aligned}
|\operatorname{MFF}(r, s, p, \kappa)\rangle^{+}= & E_{-\frac{1}{2}}^{2}\left(F_{0}^{12}\right)^{r+(s-1)(\kappa+1)} F_{\frac{1}{2}}^{2} \cdot\left(E_{-1}^{12}\right)^{r+(s-2)(\kappa+1)} \times \cdots \\
& \cdots \times E_{-\frac{1}{2}}^{2}\left(F_{0}^{12}\right)^{r-(s-1)(\kappa+1)} F_{\frac{1}{2}}^{2}\left|p, \frac{r}{2}-\frac{s-1}{2}(\kappa+1), \kappa\right\rangle .
\end{aligned}
$$

Как мы отмечали, отбрасывание в формулах $М \Phi \Phi$ некоторого числа множителей слева дает состояния, по-прежнему удовлетворяюшие твистованным условиям старшего веса (но, вообще говоря, с комплексным параметром $\theta$ ) и не являющиеся элементами 
исходного модуля Верма. Пусть $j_{i}$, где $i \geq 1,-$ спин такого состояния (собственное значение оператора $\left.H_{0}^{-}\right)$, получаемого, когда в формуле для $\mathrm{MFF}^{+}$остается $i-1$ степенных множителей, действуюших на вектор старшего веса:

$$
j_{i}=\left\{\begin{array}{lll}
\frac{r}{2}-\frac{s-i}{2}(\kappa+1), & i \text { нечетное } \\
-\frac{r}{2}+\frac{s-i+1}{2}(\kappa+1), & i \text { четное. }
\end{array}\right.
$$

Модифищированные условия старшего веса (2.32) оказываются неэквивалентными стандартным, как только $p-\frac{\kappa}{2} \pm j=0$, что действительно происходит с одним из $j_{i}$. Внутри "продолженной" формулы само по себе выполнение этого условия не означает

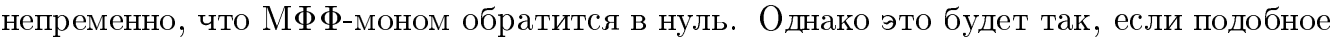
условие выполнено одновременно и “внутри” другой формулы (3.65) для того же сингулярного вектора. Это случается для $j_{i_{1}}$ и $j_{i_{2}}$, когда $i_{1}$ и $i_{2}$ одновременно оба четные или оба нечетные. Пусть для определенности $i_{1}=2 m-1$ и $i_{2}=2 n+1$, где $1 \leq m \leq n \leq s$. Тогда

$$
\kappa+1=\frac{r}{s-m-n},
$$

и, таким образом, МФФ-моном имеет следующую структуру (мы для краткости опускаем вектор старшего веса):

$$
\begin{aligned}
& \cdots \underbrace{E_{-\frac{1}{2}}^{2}\left(F_{0}^{12}\right)^{\frac{r(n+1-m)}{s-m-n}} F_{\frac{1}{2}}^{2}}_{(2 n+1)} \cdot\left(E_{-1}^{12}\right)^{\frac{r(n-m)}{s-m-n}} \times \cdots \\
& \quad \cdots \times\left(E_{-1}^{12}\right)^{\frac{r(m-n)}{s-m-n}} \cdot \underbrace{E_{-\frac{1}{2}}^{2}\left(F_{0}^{12}\right)^{\frac{r(m-n-1)}{s-m-n}} F_{\frac{1}{2}}^{2}}_{(2 m-1) \text { th }} \cdot \ldots \cdot E_{-\frac{1}{2}}^{2}\left(F_{0}^{12}\right)^{\frac{r(1-m-n)}{s-m-n}} F_{\frac{1}{2}}^{2},
\end{aligned}
$$

здесь $(2 n+1)$-й, ..., $(2 m-1)$-й множители образуют “сингулярньй вектор" $\mathrm{MFF}^{+}\left(0, S, p=\frac{(r+1) m+(1-r) n-s}{2(s-m-n)}, \kappa=\frac{r}{s-m-n}-1\right)$, где $S=n-m+2$. Такой вектор вычисляется, как указано в (3.64), что и приводит к нулевому результату в силу имеюшихся значений параметров. Анализируя подобным же образом второй случай, мы приходим к следуюшей теореме.

ТЕОрема 3.17. Массивные сингулярные векторы $\mathrm{MFF}^{ \pm}(r, s, p, \kappa)(3.62)$ обращаются в нуль при $(p, \kappa)=(\mathbf{p}(r, s, m, n), \mathbf{k}(r, s, m, n))$, где

$$
\begin{aligned}
& \mathbf{k}(r, s, m, n)+1=\frac{r}{s-m-n}, \\
& 2(s-m-n)
\end{aligned}, \quad\left\{\begin{array}{l}
1 \leq m \leq s \\
0 \leq n \leq s-1 \\
m+n \neq s \\
s \geq 2
\end{array}\right.
$$

Заметим, что при выполнении (3.67) топологические условия $p \mp j-\frac{1}{2} \kappa=0$ действительно выполняются для одного из “усеченных" МФФ-состояний, поскольку одно из чисел:

$$
p-\frac{\kappa}{2}-j_{i}=\left\{\begin{array}{ll}
\frac{r(2 m-i-1)}{2(s-m-n)}, & i \text { нечетное, } \\
\frac{r(i-2 n-2)}{2(s-m-n)}, & i \text { четное, }
\end{array} \quad p-\frac{\kappa}{2}+j_{i}= \begin{cases}\frac{r(i-2 n-1)}{2(s-m-n)}, & i \text { нечетное, } \\
\frac{r(2 m-i)}{2(s-m-n)}, & i \text { четное, }\end{cases}\right.
$$

обрашается в нуль, когда $i$ пробегает значения от 1 до $2 s-1$. 
Сингулярные векторы в точках (3.67) можно определить следующим образом:

$$
\begin{aligned}
& |\operatorname{mff}(r, s, m, n, \alpha)\rangle^{ \pm}= \\
& \quad=\lim _{\epsilon \rightarrow 0}\left(\frac{1}{\epsilon}|\operatorname{MFF}(r, s, \mathbf{p}(r, s, m, n)+\epsilon \cos \alpha, \mathbf{k}(r, s, m, n)+\epsilon \sin \alpha)\rangle^{ \pm}\right)= \\
& \quad=\left.\frac{\partial}{\partial \epsilon}\left(|\operatorname{MFF}(r, s, \mathbf{p}(r, s, m, n)+\epsilon \cos \alpha, \mathbf{k}(r, s, m, n)+\epsilon \sin \alpha)\rangle^{ \pm}\right)\right|_{\epsilon=0}
\end{aligned}
$$

Результат оказывается зависяшим от $\alpha$, и, таким образом (как в |\rangle$^{+}-$, так и в |\rangle$^{-}$-случае), имеются $\partial в а$ линейно независимых сингулярных вектора с идентичными квантовыми числами ${ }^{6)}$.

\section{CоответСтвиE $\widehat{s l}(2 \mid 1) \longleftrightarrow N=2$}

Как мы уже отмечали, гамильтонова редукция аффинной супералгебры $\widehat{s l}(2 \mid 1)$ дает $N=2$ суперконформную алгебру $[20,30,31]$. В настоящем разделе мы обсуждаем в известном смысле обратную конструкцию, позволяюшую построить представления $\widehat{s l}(2 \mid 1)$ из представлений $N=2$ алгебры и некоторых свободных конформных полей, а затем используем эту конструкцию для изучения соответствия между сингулярными векторами в $\widehat{s l}(2 \mid 1)$ и $N=2$ модулях Верма.

4.1. Построение $\widehat{s l}(2 \mid 1)$-генераторов и пространства представления. Алгебра $\widehat{s l}(2 \mid 1)$ допускает конструкцию в терминах произвольной $N=2$ суперконформной материи (т.е. токов $\mathcal{T}(z)=\sum_{n \in \mathbb{N}} \mathcal{L}_{n} z^{-n-2}, \quad \mathcal{G}(z)=\sum_{n \in \mathbb{N}} \mathcal{G}_{n} z^{-n-2}$, $\mathcal{Q}(z)=\sum_{n \in \mathbb{N}} \mathcal{Q}_{n} z^{-n-1}$ и $\mathcal{H}(z)=\sum_{n \in \mathbb{N}} \mathcal{H}_{n} z^{-n-1}$, соответствуюших генераторам (2.13)) двух свободных бозонных токов противоположной сигнатуры и свободного фермиона $\psi \bar{\psi}$ с операторными произведениями

$$
\partial F(z) \partial F(w)=\frac{-\kappa / 2}{(z-w)^{2}}, \quad \partial U(z) \partial U(w)=\frac{\kappa / 2}{(z-w)^{2}}, \quad \psi(z) \bar{\psi}(w)=\frac{1}{z-w} .
$$

ТЕОРема 4.1. Пусть центральный заряд алгебры $N=2$ равен $\mathbf{c}=-3-6 \kappa$, $\kappa \neq 0$, и свободные поля $\partial F, \partial U$ и $\psi, \bar{\psi}$ удовлетворяют операторным произведениям (4.1). Тогда токи

$$
\begin{aligned}
E^{1}=\psi e^{\frac{1}{\kappa}(U-F)}, \quad E^{2}=\bar{\psi} e^{\frac{1}{\kappa}(U-F)}, \quad E^{12}=e^{\frac{2}{\kappa}(U-F)}, \\
H^{+}=-\frac{1}{2} \mathcal{H}+\frac{1}{2} \psi \bar{\psi}, \quad H^{-}=\partial U, \\
F^{1}=\left(\mathcal{G}-\bar{\psi} \partial F-\frac{1}{2} \mathcal{H} \bar{\psi}-\left(\kappa+\frac{1}{2}\right) \partial \bar{\psi}\right) e^{-\frac{1}{\kappa}(U-F)}, \\
F^{2}=\left(\frac{1}{2}(\kappa+1) \mathcal{Q}+\psi \partial F-\frac{1}{2} \mathcal{H} \psi+\left(\kappa+\frac{1}{2}\right) \partial \psi\right) e^{-\frac{1}{\kappa}(U-F)}, \\
F^{12}=\left(-\partial F \partial F-(\kappa+1) \partial \partial F+(\kappa+2) T_{N=0}\right) e^{-\frac{2}{\kappa}(U-F)},
\end{aligned}
$$

\footnotetext{
${ }^{6)}$ Кратность $\widehat{s l}(2 \mid 1)$-сингулярных векторов была впервые отмечена в [41].
} 
$2 \partial e$

$$
\begin{aligned}
T_{N=0}= & \frac{1}{\kappa+2}\left((\kappa+1)\left(\mathcal{T}+\frac{1}{2} \partial \mathcal{H}\right)+\frac{1}{4} \mathcal{H} \mathcal{H}+\mathcal{G} \psi-\frac{1}{2}(\kappa+1) \mathcal{Q} \bar{\psi}+\frac{1}{2} \mathcal{H} \psi \bar{\psi}+\right. \\
& \left.\left.+\frac{1}{4}(1+2 \kappa) \psi \partial \bar{\psi}-\frac{1}{4}(1+2 \kappa) \partial \psi \bar{\psi}\right)\right),
\end{aligned}
$$

удовлетворяют алгебре $\widehat{s l}(2 \mid 1)$ уровня $\left.\kappa^{7}\right)$.

Отметим, что при подстановке $T_{N=0}$ в выражение для $F^{12}$ последнее остается хорошо определенным и при $\kappa=-2$ (вообще, $F^{12}$ определяется токами $F^{1}$ и $F^{2}$ ). Заметим также, что (4.2) не является реализацией в терминах свободных полей, коль скоро $N=$ 2 "материя" не является свободной.

Подобную же конструкцию для $\widehat{s l}(2 \mid 1)$-токов можно осуществить и в терминах токов $\widehat{s l}(2)$-алгебры уровня $k=-\frac{2 \kappa+1}{\kappa+1}, B C$-системы, свободного скаляра с положительной сигнатурой и $\psi \bar{\psi}$-системы. Рецепт построения сводится к использованию в приведенных вьше формулах отображения $\mathrm{KC}$, рассматриваемого в следуюшем разделе.

Ряд свойств $\widehat{s l}(2 \mid 1)$-алгебры весьма естественно реализуется в терминах конструкции $(4.2)$.

Лемма 4.2. Отображение спектрального потока на $\widehat{s l}(2 \mid 1)$-генераторах, построенных в теореме 4.1, реализуется как

$$
\begin{aligned}
\mathcal{L}_{n} \mapsto \mathcal{L}_{n}+\theta \mathcal{H}_{n}-\left(\kappa+\frac{1}{2}\right)\left(\theta^{2}+\theta\right) \delta_{n, 0}, \quad \mathcal{H}_{n} \mapsto \mathcal{H}_{n}-(2 \kappa+1) \theta \delta_{n, 0}, \\
\mathcal{Q}_{n} \mapsto \mathcal{Q}_{n-\theta}, \quad \mathcal{G}_{n} \mapsto \mathcal{G}_{n+\theta}, \\
\psi_{n} \mapsto \psi_{n-\theta}, \quad \bar{\psi}_{n} \mapsto \bar{\psi}_{n+\theta},
\end{aligned}
$$

где первые две строчки представляют собой $N=2$ спектральный поток (2.16).

Подобным же образом (при $\kappa \neq-1$ ) реализуется и автоморфизм $\widehat{s l}(2 \mid 1)$-алгебры $(2.26)$

$$
\begin{gathered}
\mathcal{G} \mapsto-\frac{\kappa+1}{2} \mathcal{Q}, \quad \mathcal{Q} \mapsto-\frac{2}{\kappa+1} \mathcal{G}, \quad \mathcal{H} \mapsto-\mathcal{H}, \\
\psi \mapsto \bar{\psi}, \quad \bar{\psi} \mapsto \psi,
\end{gathered}
$$

где первая строчка есть инволютивный автоморфизм $N=2$ алгебры.

Теперь мы обратимся к построению пространства представления алгебры $\widehat{s l}(2 \mid 1)$ исходя из модуля Верма над $N=2$ алгеброй. Мы фиксируем конкретный вакуум из семейства $\widehat{s l}(2 \mid 1)$-состояний старшего веса, связанных отображением спектрального потока, а именно рамоновский вакуум $\left|p, j, \kappa ; \frac{1}{2}\right\rangle$, для которого условия старшего веса (2.28) принимают вид

$$
\begin{gathered}
E_{\geq 0}^{1}\left|p, j, \kappa ; \frac{1}{2}\right\rangle=0, \quad E_{\geq 0}^{2}\left|p, j, \kappa ; \frac{1}{2}\right\rangle=0, \quad E_{\geq 0}^{12}\left|p, j, \kappa ; \frac{1}{2}\right\rangle=0, \\
F_{\geq 1}^{1}\left|p, j, \kappa ; \frac{1}{2}\right\rangle=0, \quad F_{\geq 1}^{2}\left|p, j, \kappa ; \frac{1}{2}\right\rangle=0, \quad F_{\geq 1}^{12}\left|p, j, \kappa ; \frac{1}{2}\right\rangle=0, \\
H_{0}^{+}\left|p, j, \kappa ; \frac{1}{2}\right\rangle=\left(p-\frac{\kappa}{2}\right)\left|p, j, \kappa ; \frac{1}{2}\right\rangle, \quad H_{0}^{-}\left|p, j, \kappa ; \frac{1}{2}\right\rangle=j\left|p, j, \kappa ; \frac{1}{2}\right\rangle
\end{gathered}
$$

\footnotetext{
${ }^{7)}$ Кратные нормальные произведения вычисляются справа налево, как :A:BC: .. Однако для сокрашения формул мы вынесли вертексные операторы $\exp (a(U-F))$ за скобки. Таким образом, в равенствах (4.2) следует раскрыть скобки, после чего понимать произведения как указанное нормальное произведение.
} 
(при этом подразумеваются и условия $H_{\geq 1}^{+} \approx 0, H_{\geq 1}^{-} \approx 0$, нечувствительные к спектральному потоку).

Рамоновский вакуум оказывается возможным построить как тензорное произведение некоторого $N=2$ состояния старшего веса и вакуумов свободных полей, а именно как

$$
|h, l, \kappa\rangle_{N=2} \otimes\left|e^{a(U-F)}\right\rangle \otimes|0\rangle_{\psi \bar{\psi}},
$$

где предстоит еше правильно выбрать свободные параметры. В этом разделе мы будем отличать состояния из $N=2$ модулей явным указанием |\rangle$_{N=2}$, тогда как просто кет |\rangle будет относиться к $\widehat{s l}(2 \mid 1)$. Это поможет нам обойти еше одну сложность с обозначениями: $N=2$ состояния, обозначаемые ранее как $|h, \ell, t\rangle$, должны параметризовываться в терминах $\widehat{s l}(2 \mid 1)$-уровня $\kappa$ как $\left|h, \ell, \frac{1}{\kappa+1}\right\rangle$. Мы будем записывать это жсе состояние как $|h, \ell, \kappa\rangle_{N=2}$, что позволит заодно не загромождать обозначения знаменателями. Фермионньй вакуум $|0\rangle_{\psi \bar{\psi}}$ в (4.7) определен формулами $\psi_{\geq 1}|0\rangle_{\psi \bar{\psi}}=\bar{\psi}_{\geq 0}|0\rangle_{\psi \bar{\psi}}=0$ для $\psi$ и $\bar{\psi}$ с иелочисленными модами. С некоторой долей злоупотребления $\left|e^{a(U-F)}\right\rangle$ обозначает примарное состояние, соответствующее вертексному оператору $e^{a(U-F)}$ (как легко видеть, для того чтобы удовлетворить $\widehat{s l}(2 \mid 1)$-условиям старшего веса относительно токов (4.2), необходимо, чтобы поля $U$ и $F$ входили в вертексный оператор в комбинации $U-F)$.

Еще один (дискретный) параметр доставляется возможностью выбора различных фермионных вакуумов [42] в секторе полей $\bar{\psi} \psi$, но этот параметр, как мы видели, обслуживает в точности спектральный поток на $\widehat{s l}(2 \mid 1)$, а потому он "заморожен", коль скоро мы рассматриваем $\widehat{s l}(2 \mid 1)$-состояния старшего веса в фиксированном (рамоновском) секторе.

ЛЕмма 4.3. Состояние (4.7) удовлетворяет $\widehat{s l}(2 \mid 1)$-условиям старшего веса (4.6), если и только если его $U(1)$-заряд есть $h=\kappa-2 p$, а $N=2$ размерность равна $l=\mathbf{l}(p, j, \kappa)$, где $j=k a / 2 u$

$$
\lambda(p, j, \kappa)=-\frac{\left(j+p-\frac{\kappa}{2}\right)\left(1-j+\frac{\kappa}{2}+p\right)}{1+\kappa} .
$$

Таким образом, $\widehat{s l}(2 \mid 1)$-состояние старшего веса в рамоновском секторе имеет вид

$$
\left|p, j, \kappa ; \frac{1}{2}\right\rangle=|\kappa-2 p, \lambda(p, j, \kappa), \kappa\rangle_{N=2} \otimes\left|e^{2 j / \kappa(U-F)}\right\rangle \otimes|0\rangle_{\psi \bar{\psi}} .
$$

Применяя к обеим частям (4.9) соответствуюшие отображения спектрального потока, мы получим подобным же образом все скрученные $\widehat{s l}(2 \mid 1)$-состояния старшего веса $|\tilde{p}, j, \kappa ; \theta\rangle$.

Пусть теперь над $N=2$ состоянием $|h, l, \kappa\rangle_{N=2}$ существует заряженный сингулярньй вектор (3.45). Это означает, что размерность $l$ этого состояния есть $l=$ $\lambda_{\mathrm{ch}}(r, h, \kappa) \equiv \ell_{\mathrm{ch}}\left(r, h, \frac{1}{\kappa+1}\right)$, где $\ell_{\mathrm{ch}}$ дается формулой $(3.44) ;$ полагая еще $h=\kappa-2 p$, мы, таким образом, имеем $N=2$ состояние $|\kappa-2 p, r[(r-1)(\kappa+1)-2 p+\kappa], \kappa\rangle_{N=2}$, которое мы собираемся одеть в $\widehat{s l}(2 \mid 1)$-состояние старшего веса в соответствии с рецептом леммы 4.3. Это возможно сделать ровно двумя способами, даюшими состояния вида (4.9) со следующими значениями спина $j$ :

$$
j=p-\frac{\kappa}{2}+(1-r)(\kappa+1), \quad \text { или } \quad j=-p+\frac{\kappa}{2}+r(\kappa+1) ;
$$


выбор $j$ в качестве независимой переменной тогда воспроизводит соотношения (3.56):

$p=\mathbf{p}_{1}(1-r, j, \kappa)=j+\frac{\kappa}{2}+(r-1)(\kappa+1) \quad$ или $\quad p=\mathbf{p}_{2}(-r, j, \kappa)=-j+\frac{\kappa}{2}+r(\kappa+1)$.

Таким образом, каждое из получаюшихся $\widehat{s l}(2 \mid 1)$-состояний старшего веса допускает сушествование заряженного $\widehat{s l}(2 \mid 1)$-сингулярного вектора.

Ситуация оказывается аналогичной и при одевании $N=2$ состояния старшего веса, над которым существует массивный $N=2$ сингулярный вектор; такие состояния имеют $\mathcal{L}_{0}$-размерность $(3.46)$ и в нынешних обозначениях записываются как $|\kappa-2 p, \lambda(r, s, \kappa-2 p, \kappa), \kappa\rangle_{N=2}$, где $\lambda(r, s, h, \kappa)=\ell\left(r, s, h, \frac{1}{\kappa+1}\right)$. Как легко видеть, каждое состояние старшего веса $|\kappa-2 p, \ell(r, s, \kappa-2 p, \kappa), \kappa\rangle_{N=2}, r, s \in \mathbb{N}$, может быть одето в $\widehat{s l}(2 \mid 1)$-состояние старшего веса в рамоновском секторе в точности двумя способами, дающими состояния вида (4.9) со следующими значениями спина $j$ :

$$
j=\mathbf{j}^{-}(s, r+1, \kappa)=-\frac{1}{2} s+\frac{1}{2}(r+1)(\kappa+1), \quad j=\mathbf{j}^{+}(s, r, \kappa)=\frac{1}{2} s-\frac{1}{2}(r-1)(\kappa+1) .
$$

Таким образом, каждое из получающихся $\widehat{s l}(2 \mid 1)$-состояний старшего веса допускает сушествование массивного $\widehat{s l}(2 \mid 1)$-сингулярного вектора. Важно заметить, что, коль скоро мы имеем $r, s=1,2, \ldots$ в (3.46), формула (4.12) воспроизводит все случаи из (3.61), кроме $\mathbf{j}^{-}(n, 1, \kappa), n \geq 1$.

4.2. $\widehat{s l}(2 \mid 1)$-сингулярные векторы в терминах $N=2$ алгебры. Приведенные конструкции подсказывают, что не только состояния старшего веса в $\widehat{s l}(2 \mid 1)$ и $N=2$ алгебрах соответствуют друг другу, но что подобное же соответствие должно иметься и между сингулярными векторами в соответствуюших модулях Верма. Мы переходим теперь к выводу явного соответствия между $\widehat{s l}(2 \mid 1)$ и $N=2$ сингулярными векторами.

Заряженные сингулярные векторы. По-прежнему фиксируя рамоновский сектор $\widehat{s l}(2 \mid 1)$-состояний, рассмотрим заряженные сингулярные векторы $|E(r, j, \kappa)\rangle_{\mathrm{ch}}^{(i),(\mathrm{R})}=\mathcal{U}_{\frac{1}{2}}|E(r, j, \kappa)\rangle_{\mathrm{ch}}^{(i)}$, где $\mathcal{U}_{\theta}$ обозначает отображение спектрального потока (2.27).

ТЕОРема 4.4. Заряженные-ІІ сингулярнье векторы (3.57), отображсеннье в рамоновский сектор, вычисляются в реализачии (4.2), (4.9) как заряэсенные $N=2$ сингулярные векторы (3.45), тензорно умножсенные на вакуумы свободных полей:

$$
|E(r, j, \kappa)\rangle_{\mathrm{ch}}^{(2),(\mathrm{R})}=\left\{\begin{aligned}
\mathcal{G}_{r} \ldots & \mathcal{G}_{-1} \times \\
& \times\left|\kappa-2 \mathbf{p}_{2}(r, j, \kappa), \lambda_{\mathrm{ch}}\left(-r, \kappa-2 \mathbf{p}_{2}(r, j, \kappa), \kappa\right), \kappa\right\rangle_{N=2} \otimes \\
& \left.\otimes\left|e^{2 j / \kappa(U-F)\rangle} \otimes \bar{\psi}_{r} \ldots \bar{\psi}_{-1}\right| 0\right\rangle_{\psi \bar{\psi}}, \quad r \leq-1, \\
(-1)^{r}\left(\frac{\kappa+1}{2}\right)^{r+1} \mathcal{Q}_{-r} \ldots \mathcal{Q}_{0} \times & \\
\times & \left|\kappa-2 \mathbf{p}_{2}(r, j, \kappa), \lambda_{\mathrm{ch}}\left(-r, \kappa-2 \mathbf{p}_{2}(r, j, \kappa), \kappa\right), \kappa\right\rangle_{N=2} \otimes \\
\otimes & \left.\left|e^{2 j / \kappa(U-F)\rangle} \otimes \psi_{-r+1} \ldots \psi_{0}\right| 0\right\rangle_{\psi \bar{\psi}}, \quad r \geq 0,
\end{aligned}\right.
$$

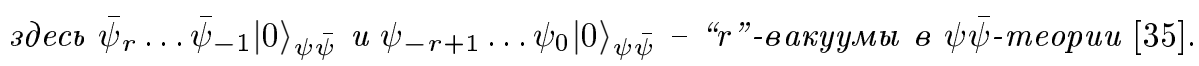


ТЕОРемА 4.4'. Заряжсенные-I сингулярные векторы (3.59), отображсенне в рамоновский сектор, вычисляются в терминах реализации (4.2), (4.9) как заряженные $N=2$ сингулярные векторы (3.45), тензорно умноженные на вакуумы свободных полей:

$$
|E(r, j, \kappa)\rangle_{\mathrm{ch}}^{(1),(\mathrm{R})}=\left\{\begin{aligned}
&(-1)^{r} \mathcal{G}_{r-1} \ldots \mathcal{G}_{-1} \times \\
& \times\left|-2 \mathbf{p}_{1}(r, j, \kappa), \lambda_{\mathrm{ch}}\left(1-r, \kappa-2 \mathbf{p}_{1}(r, j, \kappa), \kappa\right), \kappa\right\rangle_{N=2} \otimes \\
& \otimes\left|e^{2 j / \kappa(U-F)}\right\rangle \otimes \bar{\psi}_{r} \ldots \bar{\psi}-1|0\rangle_{\psi \bar{\psi}}, \quad r \leq 0, \\
&\left(\frac{\kappa+1}{2}\right)^{r} \mathcal{Q}_{-r+1} \ldots \mathcal{Q}_{0} \times \\
& \times\left|-2 \mathbf{p}_{1}(r, j, \kappa), \lambda_{\mathrm{ch}}\left(1-r,-2 \mathbf{p}_{1}(r, j, \kappa), \kappa\right), \kappa\right\rangle_{N=2} \otimes \\
& \otimes\left|e^{2 j / \kappa(U-F)}\right\rangle \otimes \psi_{-r+1} \ldots \psi_{0}|0\rangle_{\psi \bar{\psi}}, \quad r \geq 1 .
\end{aligned}\right.
$$

Интересуясь только $N=2$ составляюшей $\widehat{s l}(2 \mid 1)$-сингулярных векторов и опуская, таким образом, $U F$ - и $\psi \bar{\psi}$-секторы, мы будем иметь следующие редукиии заряженных $\widehat{s l}(2 \mid 1)$-сингулярных векторов:

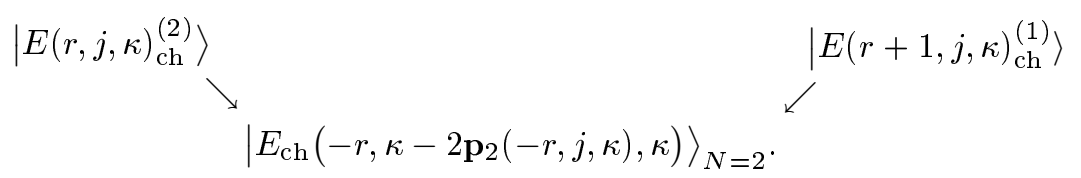

Массивные сингулярные векторы. Обратимся теперь к массивным $\widehat{s l}(2 \mid 1)$-сингулярным векторам, даваемым конструкцией $М \Phi \Phi$-типа (3.62). Как и раньше, ${ }^{(\mathrm{R})}$ будет указывать на рамоновский сектор. Начнем со специального случая $\left|\mathrm{MFF}^{-}(r, 1, p, \kappa)\right\rangle$, отмеченного после формулы (4.12).

ТеОРема 4.5. Сингулярные векторы $\left|\mathrm{MFF}^{-}(r, 1, p, \kappa)\right\rangle$, вычисленные в реализаиии (4.2), (4.9), принимают вид

$$
\begin{aligned}
\left|\mathrm{MFF}^{-}(r, 1, p, \kappa)\right\rangle^{(\mathrm{R})}=\mid \kappa & \left.-2 p, \frac{1}{\kappa+1}\left(\frac{r-1}{2}-p\right)\left(\frac{r+1}{2}+p\right), \kappa\right\rangle_{N=2} \otimes \\
& \otimes\left|e^{\frac{r+\kappa+1}{\kappa}(U-F)}\right\rangle \otimes|0\rangle_{\psi \bar{\psi}}, \quad r \geq 1 .
\end{aligned}
$$

В терминах $N=2$ алгебры, таким образом, эти $\widehat{s l}(2 \mid 1)$-сингулярные векторы редуцируются к состояниям старшего веса, а не к сингулярным векторам.

Оставшиеся массивные сингулярные векторы $\widehat{s l}(2 \mid 1)$-алгебры мы будем сравнивать с нейтральными $N=2$ сингулярными векторами. В случае общего положения последние даются любым из двух выражений:

$$
\begin{aligned}
|S(r, s, h, \kappa)\rangle & =\mathcal{N}_{1}(r, s, h, \kappa) g(0,-r s-1)\left|S\left(r, s, h, \frac{1}{\kappa+1}\right)\right\rangle^{-}= \\
& =\mathcal{N}_{2}(r, s, h, \kappa) q(1,-r s)\left|S\left(r, s, h, \frac{1}{\kappa+1}\right)\right\rangle^{+},
\end{aligned}
$$

где действие операторов отрицательный целой длины на состояния (3.47) определено в соответствии с описанными в пункте 3.2 рецептами алгебраического продолжения, а 
соответствующие нормировочные множители равны

$$
\begin{aligned}
& \mathcal{N}_{1}(r, s, h, \kappa)=\prod_{n=1}^{r}\left(h-\eta^{+}\left(r, s, 1-n, \frac{1}{\kappa+1}\right)\right), \\
& \mathcal{N}_{2}(r, s, h, \kappa)=\prod_{n=1}^{r}\left(h-\eta^{-}\left(r, s, n, \frac{1}{\kappa+1}\right)\right),
\end{aligned}
$$

где $\eta^{+}(r, s, p, t)=s+\frac{-r+1-2 p}{t}, \eta^{-}(r, s, p, t)=-s+\frac{r+1-2 p}{t}$.

Когда оба нормировочных множителя (4.18) обрашаются в нуль, что происходит при

$$
\begin{gathered}
h=\frac{(1-m+n) s}{-m-n+r}, \quad \kappa+1=\frac{s}{r-m-n}, \quad 0 \leq n \leq r-1, \\
1 \leq m \leq r, \quad m+n-r \neq 0, \quad r \geq 2,
\end{gathered}
$$

то мы имеем вместо (4.17) в точности кратные нейтральные сингулярные векторы

$$
\begin{aligned}
|\mathbf{s}(r, s, m, n)\rangle^{-}= & \left(\prod_{i=0}^{-m+r-1} \mathcal{G}_{i}\right) \mathcal{E}^{-,-m}\left(r, s, t=\frac{r-m-n}{s}\right) \times \\
& \times\left(\prod_{j=-m}^{-1} \mathcal{G}_{j}\right)\left|\frac{(1-m+n) s}{r-m-n}, \frac{m n s}{r-m-n}, \kappa=\frac{s}{r-m-n}-1\right\rangle_{N=2}, \\
|\mathbf{s}(r, s, m, n)\rangle^{+}= & \left(\prod_{i=1}^{-n+r-1} \mathcal{Q}_{i}\right) \mathcal{E}^{+, n}\left(r, s, t=\frac{r-m-n}{s}\right) \times \\
& \times\left(\prod_{j=-n}^{0} \mathcal{Q}_{j}\right)\left|\frac{(1-m+n) s}{r-m-n}, \frac{m n s}{r-m-n}, \kappa=\frac{s}{r-m-n}-1\right\rangle_{N=2}
\end{aligned}
$$

(здесь $\mathcal{E}^{ \pm}(r, s, t)$ - операторы топологических сингулярных векторов (3.42), (3.43), a $\mathcal{E}^{ \pm, \theta}$ обозначает действие на них отображения спектрального потока).

Относительно редукции массивных $\operatorname{sl}(2 \mid 1)$-сингулярных векторов к указанным нейтральным $N=2$ сингулярным векторам у нас нет прямого доказательства, но серия явных вычислений, “нумерология" и требования самосогласованности позволяют нам сформулировать следуюшую теорему.

TEOPEMA 4.6.

I. В случае общего положсения массивные сингулярные векторы (3.62) вычисляются в терминах $N=2$ алгебры как массивные сингулярные векторы, тензорно умножсенные на примарные состояния свободных полей:

$$
\begin{aligned}
\left|\mathrm{MFF}^{-}(r, s+1, p, \kappa)\right\rangle^{(\mathrm{R})}=(-1)^{s} 2^{r-s} r\left(\frac{\kappa+1}{2}\right)^{r s} \times & \\
\times & |S(s, r, \kappa-2 p, \kappa)\rangle_{N=2} \otimes\left|e^{\frac{r+s(\kappa+1)}{\kappa}(U-F)}\right\rangle \otimes|0\rangle_{\psi \bar{\psi}}, \\
& \times\left|\mathrm{MFF}^{+}(r, s, p, \kappa)\right\rangle^{(\mathrm{R})}=(-1)^{s-1} 2^{r+1-s} r\left(\frac{\kappa+1}{2}\right)^{r(s-1)} \times \\
\quad & \mid S, r, \kappa-2 p, \kappa)\rangle_{N=2} \otimes\left|e^{\frac{-r-(s-1)(\kappa+1)}{\kappa}(U-F)}\right\rangle \otimes|0\rangle_{\psi \bar{\psi}}, \quad r, s \geq 1 .
\end{aligned}
$$


Таким образом, за исключением векторов $\operatorname{MFF}^{-}(r, 1, p, \kappa)$ массивные $\widehat{s l}(2 \mid 1)$-сингулярные векторы (3.62) “дважды накрывают" массивные $N=2$ сингулярные векторы:

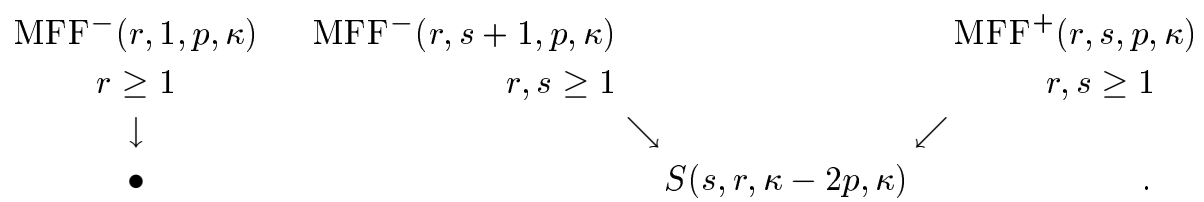

Явные вычисления, в которых проверялось это утверждение, были проведены для векторов $\mathrm{MFF}^{-}$и $\mathrm{MFF}^{+}$, соответственно, в следующих случаях:

$\begin{array}{ccccccccc}r \backslash^{s+1} & 2 & 3 & 4 & r \backslash^{s} & 1 & 2 & 3 & 4 \\ 1 & 8 / 3 & 36 / 9 & 139 / 22 & 1 & 2 / 3 & 11 / 9 & 48 / 22 & 171 / 51 \\ 2 & 33 / 9 & 442 / 51 & & 2 & 2 / 9 & 49 / 51 & & \\ 3 & 107 / 22 & & & 3 & 2 / 22 & & & \\ & & & 4 & 2 / 51 & & & \end{array}$

Здесь обозначение $m / n$ указывает на то, что явное выражение для рассматриваемого $\widehat{s l}(2 \mid 1)$-сингулярного вектора в виде элемента модуля Верма содержит $m$ слагаемых, а соответствующий $N=2$ сингулярный вектор содержит $n$ слагаемых.

В “исключительных" случаях (3.67) имеется аналогичное соответствие между двумерными пространствами линейно независимых сингулярных векторов. Сравнивая (3.67) и (4.19), мы видим, что эти множества “исключительных" точек переходят друг в друга при условии, что $r \leftrightarrow s$, но такая перестановка как раз и наблюдалась при редукции сингулярных векторов! Прямая подстановка параметров (3.67) и (4.19) в (4.21) дает равенство $0=0$, т.к. $\left.h\right|_{(4.19)}=2 \mathbf{k}(s, r, m, n)-\mathbf{p}(s, r, m, n),\left.\kappa\right|_{(4.19)}+1=\mathbf{k}(s, r, m, n)+1$. Обрашаясь к (3.69), мы будем иметь ту или иную линейную комбинацию двух сингулярных векторов в зависимости от способа приближения к точке обрашения в нуль; при этом имеется следуюшее соответствие между кратными сингулярными векторами двух алгебр:

TEOPEMA 4.6.

II. Двумерное пространство $\widehat{s l}(2 \mid 1)$-сингулярных векторов, порохденное векторами $|\operatorname{mff}(r, s, m, n, \alpha)\rangle^{+}, r \geq 1, s \geq 2$ (соответственно $|\operatorname{mff}(r, s+1, m, n, \alpha)\rangle^{-}$, $r, s, \geq 1)$, в точках (3.67), редущируется $\kappa$ двумерному пространству $N=2$ сингулярных векторов, порожденному векторами $\left|\mathbf{s}(s, r, m, n\rangle^{-} u\right| \mathbf{s}(s, r, m, n\rangle^{+}$, c.M. (4.20).

\section{5. ОТОБРАЖЕНИЕ КАДЗАМЫ-СУДЗУКИ И ЭКВИВАЛЕНТНОСТЬ КАТЕГОРИЙ $N=2$ И $\widehat{s l}(2)$-ПРЕДСТАВЛЕНИЙ}

В то время как $\widehat{s l}(2 \mid 1)$ и $N=2$ сингулярные векторы "похожи" в установленном выше смысле, подобное же сравнение $\widehat{s l}(2)$ и $N=2$ модулей демонстрирует, что не только сингулярные векторы совпадают [32], но и имеется более сильный результат - эквивалентность двух категорий представлений с точностью до соответствующих спектральных потоков. Эта эквивалентность устанавливается при помощи отображения КС, что потребует от нас введения некоторых вспомогательных конструкций.

5.1. Вспомогательные свободные поля. Введем систему свободных фермионов (так называемые духи, или $b c$-систему), определяемую операторными произведениями 
$B(z) C(w)=\frac{1}{z-w}$ и тензором энергии-импульса $T^{\mathrm{gh}}=-B \partial C$. Разложение по модам имеет вид $B(z)=\sum_{n \in \mathbb{Z}} B_{n} z^{-n-1}$ и $C(z)=\sum_{n \in \mathbb{Z}} C_{n} z^{-n}$. Пусть $\Omega$ обозначает модуль, порожденньй из любого из состояний $|\lambda\rangle, \lambda \in \mathbb{Z}$, определенных посредством

$$
C_{\geq 1-\lambda}|\lambda\rangle_{\mathrm{gh}}=B_{\geq \lambda}|\lambda\rangle_{\mathrm{gh}}=0 .
$$

Все такие вакуумы лежат в одном и том же модуле [42], и при этом состояния $|\lambda\rangle_{\mathrm{gh}} \mathrm{c}$ различными $\lambda$ связаны друг с другом операторами $c(\mu, \nu)$ и $b(\mu, \nu)$, являюшимися произведениями фермионных мод

$$
c(\mu, \nu)=\prod_{n=1}^{\nu-\mu+1} C_{\mu+n}, \quad b(\mu, \nu)=\prod_{n=1}^{\nu-\mu+1} B_{\nu+n}, \quad \nu-\mu+1 \in \mathbb{N},
$$

а именно, $c(\mu, \nu)$ и $b(\mu, \nu)$ отображают вектор $|\lambda\rangle_{\text {gh как }}$

$$
c(-\lambda-\ell+1,-\lambda):|\lambda\rangle_{\mathrm{gh}} \mapsto|\lambda+\ell\rangle_{\mathrm{gh}}, \quad b(-\lambda-\ell, \lambda-1):|\lambda\rangle_{\mathrm{gh}} \mapsto|\lambda-\ell\rangle_{\mathrm{gh}},
$$

Свойства объектов $c(\mu, \nu)$ и $b(\mu, \nu)$, такие как

$$
c(\mu, \nu-1) c\left(\nu, \nu_{1}\right)=c\left(\mu, \nu_{1}\right), \quad b(\mu, \nu-1) b\left(\nu, \nu_{1}\right)=b\left(\mu, \nu_{1}\right),
$$

могут быть продолжены на случай произвольных комплексных $\mu$ и $\nu$.

Введем также "лиувиллевский" скаляр - свободное скалярное поле, называемое так из-за “лиувиллевской" сигнатуры $\phi(z) \phi(w)=-\ln (z-w)$. Определим еще вертексные операторы - "антифермионы" - как $\psi=e^{\phi}$ и $\psi^{*}=e^{-\phi}$. Лиувиллевский тензор энергии-импульса полагается равным

$$
T_{\phi}=-\frac{1}{2} \partial \phi \partial \phi+\frac{1}{2} \partial^{2} \phi .
$$

5.2. Отображения Кадзамы-Судзуки и “анти"-Кадзамы-Судзуки. Простейшее отображение $\mathrm{KC}[27,28,43,44]$ позволяет построить генераторы $N=2$ алгебры каK

$$
\begin{gathered}
\mathcal{Q}=C J^{+}, \quad \mathcal{G}=\frac{2}{k+2} B J^{-} \\
\mathcal{H}=\frac{k}{k+2} B C-\frac{2}{k+2} J^{0}, \quad \mathcal{T}=\frac{1}{k+2}\left(J^{+} J^{-}\right)-\frac{k}{k+2} B \partial C-\frac{2}{k+2} B C J^{0} .
\end{gathered}
$$

Эти генераторы удовлетворяют алгебре (2.13) с центральным зарядом $\mathbf{c}=\frac{3 k}{k+2}$, где $k$ - уровень $\widehat{s l}(2)$. Тем самым равенства (5.6) и (5.7) задают отображение $F_{\mathrm{KS}}: \mathcal{A} \rightarrow$ $\mathcal{U} \widehat{s l}(2)_{k} \otimes[B C]$, где $\mathcal{A}$ обозначает $N=2$ алгебру (2.13) и $\mathcal{U}$ - универсальную обертывающую, а $[B C]$ - теорию свободных фермионов.

Наряду с $N=2$ генераторами имеется также коммутирующий с ними свободный ток

$$
I^{+}=\sqrt{\frac{2}{k+2}}\left(B C+J^{0}\right)
$$

моды которого, определяемые как $I^{+}(z)=\sum_{n \in \mathbb{Z}} I_{n}^{+} z^{-n-1}$, порождают алгебру Гайзенберга $\left[I_{m}^{+}, I_{n}^{+}\right]=m \delta_{m+n, 0}$. Тензор энергии-импульса этого $U(1)$-тока полагается равным

$$
T^{+}=\frac{1}{2}\left(I^{+}\right)^{2}-\frac{1}{\sqrt{2(k+2)}} \partial I^{+} .
$$

Определим модуль $\mathcal{H}_{p}^{+}$над алгеброй Гайзенберга с помощью следующих условий старшего веса: $I_{\geq 1}|p\rangle^{+}=0, I_{0}|p\rangle^{+}=p|p\rangle^{+}$. 
Лемма 5.1. При отображении КС имеем тождества

$$
T_{\mathrm{Sug}}+T_{\mathrm{gh}}=\mathcal{T}+T^{+}, \quad J^{0}-B C=-2 \mathcal{H}+\frac{k-2}{\sqrt{2(k+2)}} I^{+} .
$$

Отображение, направленное в определенном смысле в обратную сторону, строится с помошью системы “антифермионов" $\psi=e^{\phi}, \psi^{*}=e^{-\phi}$. При $N=2$ центральном заряде $\mathbf{c} \neq 3$, генераторы

$$
J^{+}=\mathcal{Q} \psi, \quad J^{-}=\frac{3}{3-\mathbf{c}} \mathcal{G} \psi^{*}, \quad J^{0}=-\frac{3}{3-\mathbf{c}} \mathcal{H}+\frac{k}{2} \partial \phi
$$

удовлетворяют алгебре (2.1) уровня $k=\frac{2 \mathbf{c}}{3-\mathbf{c}}$. Таким образом определено отображение

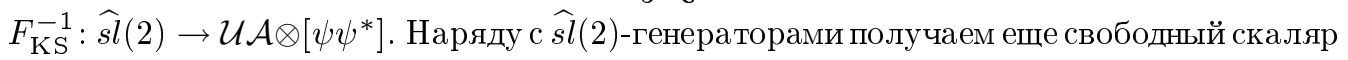

$$
I^{-}=\sqrt{\frac{3}{3-\mathbf{c}}}(H-\partial \phi)
$$

с сигнатурой -1 , моды которого, вводимые как $I^{-}(z)=\sum_{n=-\infty}^{\infty} I_{n}^{-} z^{-n-1}$, порождают

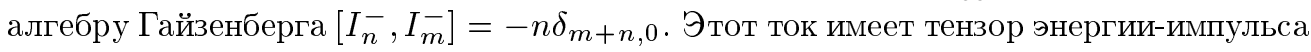

$$
T^{-}=-\frac{1}{2}\left(I^{-}\right)^{2}-\frac{1}{\sqrt{2(k+2)}} \partial I^{-} .
$$

Определим модуль $\mathcal{H}_{q}^{-}$над алгеброй Гайзенберга при помощи следующих условий старшего веса: $I_{n}^{-}|q\rangle^{-}=0, n \geq 1, I_{0}^{-}|q\rangle^{-}=q|q\rangle^{-}$.

ЛЕмма 5.2. При отображении анти-КС имеем тождества

$$
\mathcal{T}+T_{\phi}=T_{\text {Sug }}+T^{-}, \quad-2 H+\partial \phi=J^{0}+\left(\frac{k}{2}-1\right) \sqrt{\frac{k+2}{2}} I^{-} .
$$

Рассмотрим теперь композицию отображений КС и анти-КС.

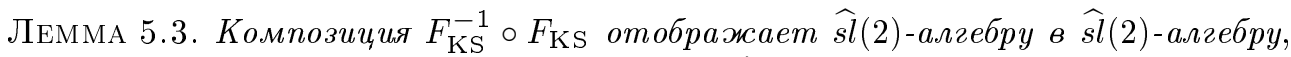
представленную в тензорном произведении $\mathcal{U} \widehat{s l}(2) \otimes\left[\psi \psi^{*}\right] \otimes[B C]$ посредством генераторов

$$
\bar{J}^{+}=J^{+} e^{\phi} C, \quad \bar{J}^{-}=J^{-} e^{-\phi} B, \quad \bar{J}^{0}=J^{0}+\frac{k}{2}(\partial \phi-B C) .
$$

Лемма 5.4. Композиция $F_{\mathrm{KS}} \circ F_{\mathrm{KS}}^{-1}$ отображсает $N=2$ алгебру в $N=2$ алгебру, представленную в тензорном произведении $\mathcal{U} \mathcal{A} \otimes\left[\psi \psi^{*}\right] \otimes[B C]$ посредством генераторов

$$
\begin{gathered}
\overline{\mathcal{Q}}=\mathcal{Q} e^{\phi} C, \quad \overline{\mathcal{G}}=\mathcal{G} e^{-\phi} B, \quad \overline{\mathcal{H}}=\mathcal{H}+\frac{k}{k+2}(B C-\partial \phi), \\
\overline{\mathcal{T}}=\mathcal{T}+\mathcal{H}(B C-\partial \phi)+\frac{k}{2(k+2)}\left((\partial \phi)^{2}-2 \partial \phi B C+\partial^{2} \phi-2 B \partial C\right) .
\end{gathered}
$$

Леммы 5.3 и 5.4 позволяют доказать следуюшие утверждения. 
TEOPEMA 5.5.

I. Отображение KC индуцирует изоморфизм $N=2$ представлений

$$
\mathfrak{R}_{j, \Lambda, k ; \theta} \otimes \Omega \approx \bigoplus_{\lambda \in \mathbb{Z}} \mathfrak{M}_{\frac{-2 j}{k+2}, \frac{\Lambda}{k+2}, k+2 ; \lambda-\theta} \otimes \mathcal{H}^{+} \sqrt{\frac{2}{k+2}}\left(j-\frac{k}{2} \theta-\lambda\right),
$$

где в левой части $N=2$ алгебра действует посредством генераторов (5.6)-(5.7), а в правой части естественно действует на $\mathfrak{M}_{\frac{-2 j}{k+2}, \ell, k+2 ; \lambda-\theta}$ как на массивном модуле Верма.

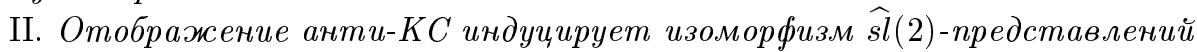

$$
\mathfrak{M}_{h, \ell, t ; \theta} \otimes \Xi \approx \bigoplus_{n \in \mathbb{Z}} \mathfrak{R}_{-\frac{t}{2} h, t \ell, t-2 ; n-\theta} \otimes \mathcal{H}^{-} \sqrt{\frac{t}{2}}\left(h-\frac{t-2}{t} \theta+n\right),
$$

где в левой части алгебра $\widehat{s l}(2)$ действует генераторами (5.11), а в правой части естественно действует на $\mathfrak{R}_{-\frac{t}{2} h, t-2 ; n-\theta}$ как на расслабленном модуле Верма.

Проиллюстрируем содержание этой теоремы на следуюшей диаграмме:

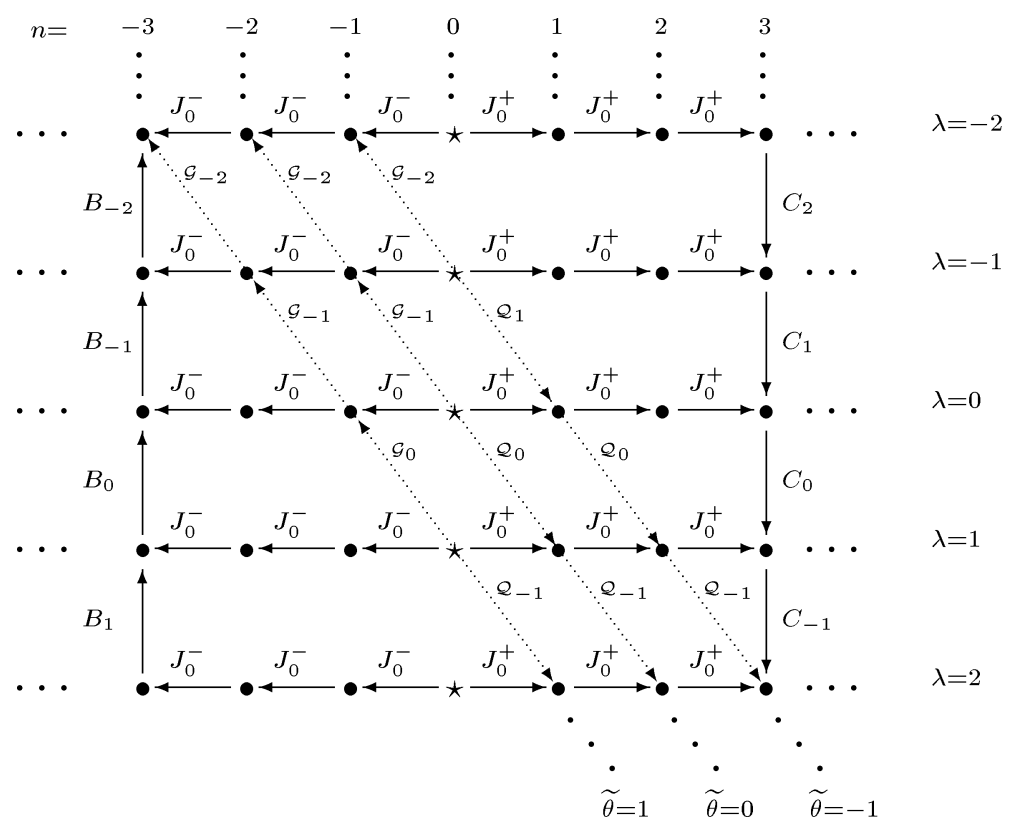

Здесь рассматривается случай, когда в (5.17) выбран “спектральный параметр” $\theta=0$ (который не следует путать со "спектральным параметром", нумерующим скрученные $N=2$ модули и обозначенным на той же диаграмме как $\widetilde{\theta}$ ). Диаграмма изображает тензорное произведение экстремальной диаграммы (2.9) расслабленного модуля Верма с духовой экстремальной диаграммой. Последняя состоит из духовых вакуумов (5.1) в различных картинах. В соответствии с выбором $\theta=0$ стрелки, соответствуюшие $\widehat{s l}(2)$, показаны горизонтальными (как и в (2.9)), а духовые ( $B$ и $C$ ) выбраны вертикальными. Последние для простоты указаны явно лишь в двух столбцах. Поскольку все картины (5.1) в теории свободных фермионов эквивалентны, каждая из вертикальных стрелок обратима, однако мы показали стрелки $B$ и $C$ в разных столбцах с тем, чтобы не 
перегружать диаграмму. Далее, точки • обозначают состояния $|j, \Lambda, k| n\rangle_{s l(2)} \otimes|\lambda\rangle_{\mathrm{gh}}$, где значения $n$ приведены в верхней строке, а значения $\lambda$ - в правом столбце. Звездочки $\star$ обозначают $|j, \Lambda, k\rangle_{s l(2)} \otimes|\lambda\rangle_{\mathrm{gh}}$. Пунктирные линии - это в точности экстремальные диаграммы (2.21) массивных модулей Верма $\mathfrak{M}_{h, \ell, t ; \tilde{\theta}}$ из правой части $(5.17)$, видимые сверху.

Имеется следующий аналог приведенной теоремы для $N=2$ топологических модулей Верма и обычных $\widehat{s l}(2)$-модулей Верма.

TEOPEMA 5.6.

I. Отображение $К С$ индуцирует изоморфизм $N=2$ представлений

$$
\mathfrak{M}_{j, k ; \theta} \otimes \Omega \approx \bigoplus_{m \in \mathbb{Z}} \mathfrak{V}_{\frac{-2 j}{k+2}, k+2 ; m-\theta} \otimes \mathcal{H}_{\sqrt{\frac{2}{k+2}}}^{+}\left(j-\frac{k}{2} \theta-m\right)
$$

где $N=2$ алгебра в левой части действует посредством генераторов (5.6), (5.7), тогда как в правой части она действует на $\mathfrak{V}_{\frac{-2 j}{k+2}, k+2 ; m-\theta}$ как на своем модуле Верма.

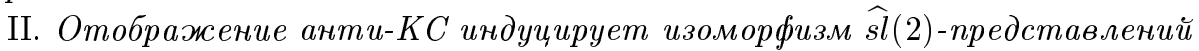

$$
\mathfrak{V}_{h, t ; \theta} \otimes \Xi \approx \bigoplus_{n \in \mathbb{Z}} \mathfrak{M}_{-\frac{t}{2} h, t-2 ; n-\theta} \otimes \mathcal{H}_{\sqrt{\frac{t}{2}}}^{-}\left(h-\frac{t-2}{t} \theta+n\right),
$$

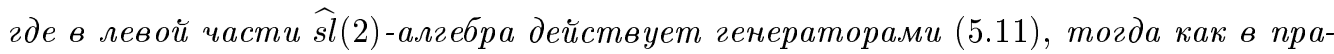
вой части она действует на $\mathfrak{M}_{-\frac{t}{2} h, t-2 ; n-\theta}$ как на своем модуле Верма.

СлЕДСТВИЕ. Установленный в теореме изоморфизм приводит к тожсдеству для характеров, которое может быть записано в виде

$$
\begin{aligned}
& \frac{\prod_{i=0}^{\infty}\left(1+z q^{i}\right) \prod_{i=1}^{\infty}\left(1+z^{-1} q^{i}\right)}{\prod_{i=0}^{\infty}\left(1-z^{-1} q^{i}\right) \prod_{i=1}^{\infty}\left(1-z q^{i}\right)}= \\
& \quad=\frac{\prod_{i=1}^{\infty}\left(1+z^{2} q^{i}\right) \prod_{i=1}^{\infty}\left(1+z^{-2} q^{i}\right)}{\prod_{i=1}^{\infty}\left(1-q^{i}\right)^{2}}\left(1+\left(1+z^{2}\right) \sum_{r=1}^{\infty}\left[\frac{z^{-r}}{1+z^{2} q^{r}}+\frac{q^{r} z^{r-2}}{1+z^{-2} q^{r}}\right]\right) .
\end{aligned}
$$

5.3. Эквивалентность цепных категорий модулей Верма. Из вышесказанного следует, что эквивалентность между некоторыми категориями $\widehat{s l}(2)$ и $N=2$ модулей может быть достигнута, только если в этих категориях эффективно осушествляется отождествление модулей, различаюшихся действием спектрального потока.

Топологический случай. Рассмотрим объекты, представляющие собой бесконечные цепи $\left(\mathfrak{M}_{j, k ; \theta}\right)_{\theta \in \mathbb{Z}}$, где $\mathfrak{M}_{j, k ; \theta}$ - скрученные модули Верма. В качестве морфиз-

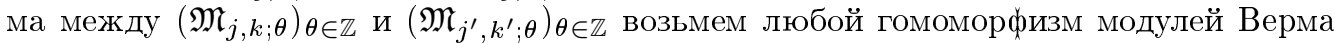
$\mathfrak{M}_{j, k ; \theta_{1}} \rightarrow \mathfrak{M}_{j^{\prime}, k^{\prime} ; \theta_{2}}$. Назовем такую категорию цепной категорией $\widehat{s l}(2)$-модулей Верма $\mathcal{C} \mathcal{V E R}$.

Со стороны $N=2$ алгебры цепная категория топологических модулей Верма $\mathcal{C} \mathcal{T} \mathcal{E} \mathcal{R}$ определяется аналогично: следует взять цепочки скрученных топологических модулей Верма, различающихся спектральным потоком на всевозможные $\theta \in \mathbb{Z}$. Морфизмы цепочек определяются аналогично $\widehat{s l}(2)$-случаю ${ }^{8)}$. Смысл определения морфизмов цепочек, конечно, в том, что, имея морфизм между двумя какими-то модулями в цепочках, мы распространяем его на всю цепочку, применяя спектральные потоки.

\footnotetext{
8) Здесь важно, что всякий подмодуль (скрученного) топологического модуля Верма - снова скрученный топологический модуль Верма или объединение двух таких модулей.
} 
Теперь мы собираемся определить функторы, связывающие категории $\widehat{s l}(2)$ и $N=2$ цепочек. Построим сначала некоторое соответствие между отдельными модулями в цепочках. Для заданного топологического модуля Верма $\mathfrak{V}_{h, t ; \theta}$ и некоторого $\theta^{\prime} \in \mathbb{Z}$ рассмотрим конструкцию

$$
\mathfrak{V}_{h, t ; \theta} \otimes \mathcal{H}_{-\sqrt{\frac{2}{t}}\left(\frac{t}{2} j+\frac{t}{2} \theta^{\prime}-\theta\right)}^{\oplus} \bigoplus_{m \in \mathbb{Z}, m \neq 0} \mathfrak{V}_{h, t ; \theta+m} \otimes \mathcal{H}_{-\sqrt{\frac{2}{t}}}^{+}\left(\frac{t}{2} j+\frac{t}{2} \theta^{\prime}-\theta+m\right),
$$

где $\mathcal{H}^{+}$
$-\sqrt{\frac{2}{t}}\left(\frac{t}{2} j+\frac{t}{2} \theta^{\prime}-\theta\right)$ - модули Гайзенберга, а $\mathfrak{V}_{h, t ; \theta+m}-$ образы заданного модуля при действии спектрального потока. Построенный объект изоморфен тензорному произведению $\widehat{s l}(2)$-модуля Верма $\mathfrak{M}_{-\frac{t}{2} h, t-2 ; \theta^{\prime}}$ на некоторьй духовьй модуль. Определим действие функтора $F_{\mathrm{KS}}\left(\theta, \theta^{\prime}\right)$ на модуль $\mathfrak{V}_{h, t ; \theta}$ как указанный модуль $\mathfrak{M}_{-\frac{t}{2} h, t-2 ; \theta^{\prime}}$ :

$$
F_{\mathrm{KS}}\left(\theta, \theta^{\prime}\right): \mathfrak{V}_{h, t ; \theta} \rightsquigarrow \mathfrak{M}_{-\frac{t}{2} h, t-2 ; \theta^{\prime}}, \quad \theta, \theta^{\prime} \in \mathbb{Z}
$$

Аналогичным образом определяется

$$
F_{\mathrm{KS}}^{-1}\left(\theta, \theta^{\prime}\right): \mathfrak{M}_{j, k ; \theta} \rightsquigarrow \mathfrak{V}_{-\frac{2}{k+2} j, k+2 ; \theta^{\prime}}, \quad \theta, \theta^{\prime} \in \mathbb{Z},
$$

для чего по заданным $\mathfrak{M}_{j, k ; \theta}$ и $\theta^{\prime} \in \mathbb{Z}$ мы строим

$$
\mathfrak{M}_{j, k ; \theta} \otimes \mathcal{H}_{-\sqrt{\frac{2}{k+2}}}^{-}\left(j+\theta^{\prime}-\frac{k+2}{2} \theta\right) \oplus \bigoplus_{n \in \mathbb{Z}, n \neq 0} \mathfrak{M}_{j+\frac{k}{2} n, k ; \theta+n} \otimes \mathcal{H}_{-\sqrt{\frac{2}{k+2}}}^{-}\left(j+\theta^{\prime}-\frac{k+2}{2} \theta+n\right)
$$

что изоморфно модулю $\mathfrak{V}_{-\frac{2}{k+2} j, k+2 ; \theta^{\prime}}$, тензорно умноженному на модуль антифермионов.

В то время как соответствия (5.23) и (5.24) зависят от выбранных $\theta$ и $\theta^{\prime}$, эта зависимость исчезает при применении к элементам $\mathcal{C} \mathcal{E} \mathcal{R}$ и $\mathcal{C} \mathcal{T} \mathcal{V} \mathcal{R}$. Таким образом, $F_{\mathrm{KS}}(\cdot, \cdot)$ и $F_{\mathrm{KS}}^{-1}(\cdot, \cdot)$ (которые мы снова обозначаем как $F_{\mathrm{KS}}$ и $F_{\mathrm{KS}}^{-1}$ ) являются кандидатами на роль функторов

$$
F_{\mathrm{KS}}: \mathcal{C} \mathcal{T} \mathcal{V} \mathcal{R} \rightsquigarrow \mathcal{C} \mathcal{V E R}, \quad F_{\mathrm{KS}}^{-1}: \mathcal{C V} \mathcal{E} \mathcal{C} \rightsquigarrow \mathcal{C} \mathcal{T} \mathcal{E} \mathcal{R}
$$

Композиция $F_{\mathrm{KS}}$ и $F_{\mathrm{KS}}^{-1}$ друг с другом отображает всякую цепь скрученных модулей Верма в изоморфную цепь. Таким образом, $F_{\mathrm{KS}}$ и $F_{\mathrm{KS}}^{-1}$ будут прямым и обратным ${ }^{9}$ функтором, как только мы выясним, что они правильно действуют на морфизмах.

В категории модулей Верма морфизмы естественно отождествляются с сингулярными векторами. Как мы видели, $\widehat{s l}(2)$-сингулярный вектор существует в скрученном модуле Верма $\mathfrak{M}_{j, k ; \theta}$ (и тем самым во всех таких модулях с $\theta \mapsto \theta+n, n \in \mathbb{Z}$ ), если и только если топологический сингулярный вектор существует хотя бы в одном скрученном топологическом модуле Верма (а тем самым и во всех таких модулях) $\mathfrak{V}_{\frac{-2 j}{k+2}, k+2 ; m}, m \in \mathbb{Z}$, из левой части соотношения (5.20). Следуюшее утверждение устанавливает явное соответствие между “строительными элементами", из которых строятся соответствующие сингулярные векторы.

\footnotetext{
9) В другой терминологии - квазиобратным, т.е. таким, композиция которого с прямым переводит каждый объект в изоморфный объект.
} 
Лемма 5.7. Отображение КС индуцирует соответствие межсду "продолженнькми" обгектами $\left(J_{-\theta}^{-}\right)^{\nu-\mu+1},\left(J_{\theta-1}^{+}\right)^{\nu-\mu+1}$, с одной стороньи, и $g(\mu, \nu), q(\mu, \nu)$, с другой сторонь, действующими на соответствующие стариие веса согласно (3.1) и (3.30). Соответствие имеет вид

$$
\begin{aligned}
& F_{\mathrm{KS}}\left(\theta^{\prime}, \theta\right): g(\mu, \nu) \mapsto\left(J_{-\theta}^{-}\right)^{\nu-\mu+1} b(\mu, \nu), \\
& F_{\mathrm{KS}}\left(\theta^{\prime}, \theta\right): q(\mu, \nu) \mapsto\left(J_{\theta-1}^{+}\right)^{\nu-\mu+1} c(\mu, \nu), \\
& F_{\mathrm{KS}}^{-1}\left(\theta, \theta^{\prime}\right):\left(J_{-\theta}^{-}\right)^{\nu-\mu+1} \mapsto g(\mu, \nu) e^{-(\nu-\mu+1) \phi}, \\
& F_{\mathrm{KS}}^{-1}\left(\theta, \theta^{\prime}\right):\left(J_{\theta-1}^{+}\right)^{\nu-\mu+1} \mapsto q(\mu, \nu) e^{(\nu-\mu+1) \phi} .
\end{aligned}
$$

Это приводит нас к следуюшему факту.

ТЕОРема 5.8. Отображения КС и анти-КС порождают отождествления, обозначаемые снова как $F_{\mathrm{KS}}\left(\theta, \theta^{\prime}\right)$ и $F_{\mathrm{KS}}^{-1}\left(\theta, \theta^{\prime}\right)$, между скрученными $\widehat{s l}(2)$-сингулярными векторами (3.4), (3.5) в модуле Верма $\mathfrak{M}_{j, k ; \theta}$ и $N=2$ сингулярными векторами (3.42) и (3.43) в скрученных топологических модулях Верма $\mathfrak{V}_{-\frac{2}{k+2} j, k+2 ; \theta}$ :

$$
\begin{aligned}
& F_{\mathrm{KS}}\left(\theta, \theta^{\prime}\right):|E(r, s, k+2)\rangle^{ \pm, \theta} \mapsto\left|\mathrm{MFF}^{ \pm}(r, s, k)\right\rangle^{\theta^{\prime}} \\
& F_{\mathrm{KS}}^{-1}\left(\theta, \theta^{\prime}\right):\left|\mathrm{MFF}^{ \pm}(r, s, k)\right\rangle^{\theta} \mapsto|E(r, s, k+2)\rangle^{ \pm, \theta^{\prime}},
\end{aligned}
$$

где $|E(r, s, t)\rangle^{ \pm, \theta} u\left|\mathrm{MFF}^{ \pm}(r, s, k)\right\rangle^{\theta^{\prime}}$ обозначают сингулярные векторы $(3.42),(3.43)$ u (3.4), (3.5), подвергнутье действию соответствующего спектрального потока.

Теперь видно, что в применении к цепочкам соответствуюших модулей Верма $F_{\mathrm{KS}}(\cdot, \cdot)$ и $F_{\mathrm{KS}}^{-1}(\cdot, \cdot)$ переводят морфизмы (между цепями) в морфизмы. Таким образом, $F_{\mathrm{KS}}$ и $F_{\mathrm{KS}}^{-1}$ действительно превращаются в функторы. Подводя итог всему сказанному, имеем следующую теорему.

ТЕОРЕМА 5.9. Функторы $F_{\mathrm{KS}}$ и $F_{\mathrm{KS}}^{-1}$ являются ковариантными взаимно обратными функторами, которые устанавливают әквивалентность между цепной ка-

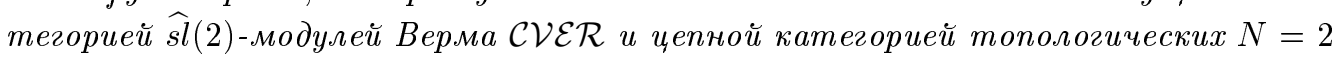
модулей Верма $\mathcal{C} \mathcal{T} \mathcal{V} \mathcal{R}$.

Расслабленный/массивный случай. Введем цепи расслабленных $\widehat{s l}(2)$-модулей Верма и цепи массивных $N=2$ модулей Верма аналогично предыдушему. Тогда имеет место следующая теорема, непосредственно вытекающая из леммы 5.7.

ТЕОрема 5.10. Отображения KC и анти-КС порождают отождествления, обозначаемые по-прежнему как $F_{\mathrm{KS}}\left(\theta, \theta^{\prime}\right)$ u $F_{\mathrm{KS}}^{-1}\left(\theta, \theta^{\prime}\right)$, между:

1) сингулярными векторами (3.9) и (3.10) в расслабленных модулях Верма $\mathfrak{R}_{j, \Lambda, k ; \theta}$ и $N=2$ сингулярнылми векторами (3.47) в скрученных массивных модулях Верма $\mathfrak{M}_{-\frac{2}{k+2} j, \frac{\Lambda}{k+2}, k+2 ; \theta}$ :

$$
\begin{aligned}
& F_{\mathrm{KS}}\left(\theta, \theta^{\prime}\right):\left|\Sigma^{ \pm}(r, s, j, k)\right\rangle^{\theta} \mapsto\left|S\left(r, s, \frac{-2 j}{k+2}, k+2\right)\right\rangle^{\mp, \theta^{\prime}} \\
& F_{\mathrm{KS}}^{-1}\left(\theta, \theta^{\prime}\right):|S(r, s, h, t)\rangle^{\mp, \theta} \mapsto\left|\Sigma^{ \pm}\left(r, s,-\frac{t}{2} h, t-2\right)\right\rangle^{\theta^{\prime}}
\end{aligned}
$$


2) а также между заряженными $\widehat{s l}(2)$-сингулярными векторами (3.7) и заряженными $N=2$ сингулярными векторами (3.45):

$$
\begin{aligned}
& F_{\mathrm{KS}}\left(\theta, \theta^{\prime}\right):|C(r, j, k)\rangle^{\theta} \mapsto|E(r, h, t)\rangle_{\mathrm{ch}}^{\theta^{\prime}}, \\
& F_{\mathrm{KS}}^{-1}\left(\theta, \theta^{\prime}\right):|E(r, h, t)\rangle_{\mathrm{ch}}^{\theta} \mapsto|C(r, j, k)\rangle^{\theta^{\prime}},
\end{aligned}
$$

где $\left|\Sigma^{ \pm}(r, s, j, k)\right\rangle^{\theta} u|S(r, s, h, t)\rangle^{\mp, \theta^{\prime}}$ u m.n. обозначают соответствующие сингулярные векторы, подвергнутые действию спектрального потока.

Теперь имеем следующую теорему

ТЕОРема 5.11. Функторы $F_{\mathrm{KS}}$ и $F_{\mathrm{KS}}^{-1}$ являются прямым и обратныцм ковариантными функторами, которые устанавливают эквивалентность иепной кате-

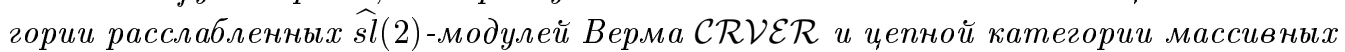
$N=2$ модулей Верма $\mathcal{C} \mathcal{M V} \mathcal{E}$.

5.4. Распространение эквивалентности на категории модулей со старшим весом. Нашей целью является распространение установленной выше эквивалентности на бо́льшие категории. Категория $\mathcal{V} \mathcal{E} \mathcal{R}$ оказывается весьма "маленькой": уже в нескрученном случае большинство интересных представлений не является модулями Верма, но получается из последних взятием факторов и склеек. В получающейся таким образом категории $\mathcal{O}$ (см. [45]) модули Верма выделены тем, что всякое неприводимое представление есть фактор-модуль некоторого модуля Верма. Стандартное определение категории $\mathcal{O}$ отбирает только нескрученные модули (с $\theta=0$ в формулах (2.5)). Замечательным фактом является то, что подобная категория модулей со старшим весом, но включающая скрученные модули, может быть определена в “инвариантных" терминах, не требуюших “перечисления” различных скручиваний категории $\mathcal{O}$. Рассмотрим сначала

ОПРЕДЕЛЕНИЕ 5.12. Пусть $|X\rangle$ - элемент модуля над $\widehat{s l}(2), \theta$ - фиксированное целое число, а $J$ обозначает $J^{+}$или $J^{-}$. Будем говорить, что $J_{\theta}$-цепь обрывается на $|X\rangle$, и писать $\left(J_{\theta}\right)^{+\infty}|X\rangle=0$, если $\exists N \in \mathbb{Z}, n \geq N:\left(J_{\theta}\right)^{n}|X\rangle=0$.

ОпредЕЛЕНиЕ 5.13. Объектами категории $\mathcal{H} \mathcal{W}$ скрученных $\widehat{s l}(2)$-представлений со старшим весом являются модули $\mathfrak{U}$ над алгеброй $\widehat{s l}(2)$, удовлетворяюшие следуюшим условиям:

1) оператор $J_{0}^{0}$ диагонализуем, т.е. $\mathfrak{U}$ разлагается в прямую сумму подпространств с фиксированными собственными значениями $J_{0}^{0}$;

2) действие операторами $J_{n}^{0}, n \in \mathbb{N}$, на всякий элемент $|X\rangle \in \mathfrak{U}$ порождает конечномерное пространство;

3) для каждого элемента $|X\rangle$ из $\mathfrak{U}$ и $\forall n \in \mathbb{Z}$ имеем или $\left(J_{n}^{+}\right)^{+\infty}|X\rangle=0$, или $\left(J_{-n}^{-}\right)^{+\infty}|X\rangle=0$. Морфизмами являются стандартные гомоморфизмы между $\widehat{s l}(2)$-модулями.

Условия обрыва цепочек означают, по существу, что экстремальная диаграмма модуля не может стать слишком открытой (на $180^{\circ}$ или более), а потому всякая прямая линия на внутренности диаграммы хотя бы одним концом попадет на экстремальное состояние, где и произойдет ее “обрыв".

Для расслабленных модулей Верма мы определим “большую” категорию подобным же образом. При этом экстремальные диаграммы могут быть открыты на $180^{\circ}$, так что предыдуший аргумент о пересечении прямой линии с экстремальной диаграммой теперь неприменим. Однако следуюшее условие исключает “переоткрытые” (более чем на $180^{\circ}$ ) диаграммы. 
ОПРЕДЕлЕниЕ 5.14. Объектами категории $\mathcal{R H \mathcal { W }}$ скрученных расслабленных $\widehat{s l}(2)$-модулей со старшим весом являются модули $U$, удовлетворяюшие следуюшим условиям:

1) оператор $J_{0}^{0}$ диагонализуем;

2) действие операторами $J_{n}^{0}, n \in \mathbb{N}$, на любой элемент $|X\rangle \in \mathfrak{U}$ порождает конечномерное пространство;

3) для всякого элемента $|X\rangle$ из $\mathfrak{U}$ и $\forall \theta \in \mathbb{Z}$ имеем или $\left(J_{\theta}^{+}\right)^{+\infty}|X\rangle=0$, или $\left(J_{-\theta+1}^{-}\right)^{+\infty}|X\rangle=0$.

Морфизмами являются стандартные гомоморфизмы между $\widehat{s l}(2)$-модулями.

Наконец, объектами категории $\mathcal{C H \mathcal { W }}$ являются цепи скрученных модулей из категории $\mathcal{H} \mathcal{W}$. Морфизмы между такими цепями определяются, как и ранее.

Аналоги этих конструкций для $N=2$ алгебры выглядят следующим образом.

ОПреДЕЛЕНИЕ 5.15. Пусть $\mathcal{F}$ обозначает $\mathcal{Q}$ или $\mathcal{G}$ (фермионные $N=2$ генераторы) и $|X\rangle$ - элемент модуля над $N=2$ алгеброй. Фиксируем $n \in \mathbb{Z}$. Будем говорить, что фермионная $\mathcal{F}$-цепь обрывается на $|X\rangle$, и писать ... $\mathcal{F}_{n-3} \mathcal{F}_{n-2} \mathcal{F}_{n-1} \mathcal{F}_{n}|X\rangle=0$, если $\exists N \in \mathbb{Z}, N \leq n: \mathcal{F}_{N} \mathcal{F}_{N+1} \ldots \mathcal{F}_{n}|X\rangle=0$.

Теперь можно дать такое определение

ОПреДЕЛЕНИЕ 5.16. Модуль $\mathfrak{U}$ над $N=2$ алгеброй является объектом топологической $N=2$ категории $\mathcal{T} \mathcal{O P}$, если:

1) операторы $\mathcal{H}_{0}$ и $\mathcal{L}_{0}$ диагонализуемы;

2) действие операторов $\mathcal{H}_{n}, n \in \mathbb{N}$, и $\mathcal{L}_{n}, n \in \mathbb{N}$, на всякий элемент $|X\rangle \in \mathfrak{U}_{\text {порож- }}$ дает конечномерное пространство;

3 для любого элемента $|X\rangle$ из $\mathfrak{U}$ и $\forall n \in \mathbb{Z}$ имеем или . . $\mathcal{Q}_{n-3} \mathcal{Q}_{n-2} \mathcal{Q}_{n-1} \mathcal{Q}_{n}|X\rangle=0$, или же $\ldots \mathcal{G}_{-n-4} \mathcal{G}_{-n-3} \mathcal{G}_{-n-2} \mathcal{G}_{-n-1}|X\rangle=0$.

Морфизмами являются стандартные гомоморфизмы между $N=2$ модулями.

Теперь мы назовем топологической цепной категорией $\mathcal{C} \mathcal{T} \mathcal{O} \mathcal{P}$ категорию, объекты которой строятся из модулей категории $\mathcal{T} \mathcal{O P}$.

Сформулированное выше условие обрыва фермионных цепочек не удовлетворяется для массивных $N=2$ модулей Верма - их экстремальные диаграммы шире топологических. Но еше более широкие цепочки не должны поместиться в диаграмме.

ОПРЕДЕлЕНИЕ 5.17. Модуль $\mathfrak{M}$ над $N=2$ алгеброй является объектом категории $\mathcal{M H \mathcal { W }}$, если:

1) операторы $\mathcal{H}_{0}$ и $\mathcal{L}_{0}$ диагонализуемы;

2) действие операторами $\mathcal{H}_{n}, n \in \mathbb{N}$, и $\mathcal{L}_{n}, n \in \mathbb{N}$, на всякий элемент $|X\rangle \in \mathfrak{U}$ порождает конечномерное пространство;

$3)$ для любого элемента $|X\rangle$ из $\mathfrak{U}$ и $\forall n \in \mathbb{Z}$ имеем или . . $\mathcal{Q}_{n-3} \mathcal{Q}_{n-2} \mathcal{Q}_{n-1} \mathcal{Q}_{n}|X\rangle=0$, или же ... $\mathcal{G}_{-n-4} \mathcal{G}_{-n-3} \mathcal{G}_{-n-2} \mathcal{G}_{-n}|X\rangle=0$.

Морфизмами являются стандартные гомоморфизмы между $N=2$ модулями.

После того как требуемые категории определены, мы можем сформулировать следующую теорему для соответствуюших цепных категорий.

ТЕОРЕМА 5.18. Функторы $F_{\mathrm{KS}}$ и $F_{\mathrm{KS}}^{-1}$ устанавливают әквивалентность между категориями $\mathcal{C H}$ и и $\mathcal{C} \mathcal{T O P}$.

Подобное же утверждение верно и для самых больших из рассматриваемых категорий $\mathcal{C} \mathcal{R} \mathcal{H} \mathcal{W}$ и $\mathcal{C} \mathcal{M} \mathcal{H} \mathcal{W}$, определяемых аналогично предыдушему. 


\section{6. ЗАКЛЮЧИТЕЛЬНЫЕ ЗАМЕЧАНИЯ}

Таким образом, модели конформной теории поля, основанные на $N=2$ суперконформной алгебре, теперь могут последовательно изучаться в терминах $\widehat{s l}(2)$-моделей ВЗНВ. Более того, как $N=2$, так и $\widehat{s l}(2)$-модели (включая и те, которые построены на "нестандартных" представлениях) должны допускать погружение в $\widehat{s l}(2 \mid 1)$-модели, состояния в которых - элементы рассмотренных выше модулей (и их факторов). Наиболее интересными представляются приложения описанных конструкций к $\widehat{s l}(2)$ и $N=2$ правилам слияния, которые должны совпадать после факторизации по спектральному потоку; интригующее исследование того, насколько они могут различаться до такой факторизации, а также вложение их в $\widehat{s l}(2 \mid 1)$-теорию, остается задачей на будущее.

Я глубоко признателен Б. Л. Фейгину и И. Ю. Типунину за плодотворное сотрудничество, а также И.В. Тютину за полезные замечания. Работа частично поддержана грантом РФФИ 96-01-00725.

\section{Список литературы}

[1] V. G. Kač, D. A. Kazhdan. Adv. Math. 1979. V. 34. P. 97.

[2] Ф. Г. Маликов, Б. Л. Фейгин, Д. Б. Фукс. Функц. анализ и его прилож. 1986. Т. 20. № 2 . C. 25.

[3] L. Benoit, Y. Saint-Aubin. Phys. Lett. B. 1987. V. 215. P. 517-522; Int. J. Mod. Phys. A. 1992. V. 7. P. 3023-3033; 1994. V. 9. P. 547-566.

[4] A. Kent. Phys. Lett. B. 1991. V. 273. P. 56-62; 1992. V. 278. P. 443-448.

[5] M. Bauer, P. di Francesco, C. Itzykson, J.-B. Zuber. Nucl. Phys. B. 1991. V. 362. P. 515-562.

[6] M. Bauer, N. Sochen. Commun. Math. Phys. 1993. V. 152. P. 127-160.

[7] A. Ch. Ganchev, V.B. Petkova. Phys. Lett. B. 1992. V. 293. P. 56-66; 1993. V. 318. P. 77-84.

[8] P. Bowcock, G. M. T. Watts. Phys. Lett. B. 1992. V. 297. P. 282-288; П. Боджок, Г. Ватmc. ТМФ. 1994. Т. 98. № 3. C. 350-356.

[9] G. M. T. Watts. Nucl. Phys. B. 1993. V. 407. P. 213-236.

[10] W. Boucher, D. Friedan, A. Kent. Phys. Lett. B. 1986. V. 172. P. 316.

[11] M. Dörrzapf. Commun. Math. Phys. 1996. V. 180. P. 195-232.

[12] A. A. Belavin, A. M. Polyakov, A. B. Zamolodchikov. Nucl. Phys. B. 1984. V. 241. P. 333.

[13] A.M. Semikhatov. Inverting the Hamiltonian Reduction in String Theory. Talk at the 28th Symposium on the Theory of Elementary Particles. Wendisch-Rietz. September 1994, hep-th/9410109.

[14] A. M. Semikhatov. Nucl. Phys. B. 1996. V. 478. P. 209.

[15] H. Awata, Y. Yamada. Mod. Phys. Lett. A. 1992. V. 7. P. 1185.

[16] B. Feigin, F. Malikov. Integral Intertwining Operators And Complex Powers Of Differential ( $q$-Difference) Operators. Kyoto preprint, RIMS-894.

[17] O. Andreev. Phys. Lett. B. 1995. V. 363. P. 166.

[18] B. Gato-Rivera, A. M. Semikhatov. Phys. Lett. B. 1992. V. 293. P. 72; Б. Гато-Pивера, A. М. Семихатов. ТМФ. 1993. Т. 95. № 2. С. 239.

[19] B. Gato-Rivera, A. M. Semikhatov. Nucl. Phys. B. 1993. V. 408. P. 133.

[20] M. Bershadsky, W. Lerche, D. Nemeschansky, N. P. Warner. Nucl. Phys. B. 1993. V. 401. P. 304-347.

[21] M. Ademollo et al. Phys. Lett. B. 1976. V. 62. P. 105; M. Ademollo et al. Nucl. Phys. B. 1976. V. 111. P. 77.

[22] N. Marcus. A Tour through $N=2$ strings. hep-th/9211059.

[23] E.S. Fradkin, A. A. Tseytlin. Phys. Lett. B. 1981. V. 106. P. 63.

[24] H. Ooguri, C. Vafa. Nucl. Phys. B. 1991. V. 361. P. 469-518; 1991. V. 367. P. 83-104.

[25] D. Kutasov, E. Martinec. New Principles for String/Membrane Unification. hep-th/9602049; D. Kutasov, E. Martinec, M. O'Loughlin. Vacua of M-theory and $N=2$ strings. hep-th/9603116.

[26] E. Martinec. Geometrical Structures of M-Theory. EFI-96-29.

[27] T. Eguchi, S. Hosono, S.-K. Yang. Commun. Math. Phys. 1991. V. 140. P. 159.

[28] W. Lerche. Phys. Lett. B. 1990. V. 252. P. 349. 
[29] A. Schwimmer, N. Seiberg. Phys. Lett. B. 1987. V. 184. P. 191

[30] M. Bershadsky, H. Ooguri. Phys. Lett. B. 1989. V. 229. P. 374.

[31] K. Ito, H. Kanno. Mod. Phys. Lett. A. 1994. V. 9. P. 1377.

[32] A. M. Semikhatov. Mod. Phys. Lett. A. 1994. V. 9. P. 1867.

[33] A. M. Semikhatov, I. Yu. Tipunin. All Singular Vectors of the $N=2$ Superconformal Algebra via the Algebraic Continuation Approach. hep-th/9604176.

[34] A. M. Semikhatov. Verma Modules, Extremal Vectors, and Singular Vectors on the Non-Critical $N=2$ String Worldsheet. hep-th/9610084.

[35] B.L. Feigin, A.M. Semikhatov, I. Yu. Tipunin. Equivalence between Categories of Verma Modules over the Affine $\widehat{s l}(2)$ and $N=2$ Superconformal Algebras. hep-th/9701043.

[36] A. M. Semikhatov, I. Yu. Tipunin. The Complete Structure of Verma Modules over the $N=2$ Superconformal Algebra. hep-th/9704111.

[37] W. Lerche, C. Vafa, N. P. Warner. Nucl. Phys. B. 1989. V. 324. P. 427.

[38] S. V. Ketov, O. Lechtenfeld. Phys. Lett. B. 1995. V. 353. P. 463-470.

[39] А. В. Стояновский, Б. Л. Фейгин. Функц. анализ и его прилож. 1994. Т. 28. № 1. С. 68

[40] A. M. Semikhatov, I. Yu. Tipunin. Int. J. Mod. Phys. A. 1996. V. 11. P. 4597.

[41] P. Bowcock, A. Taormina. Representation theory of the affine Lie superaлгебра $\operatorname{sl}(2 \mid 1)$ at fractional level. hep-th/9605220.

[42] D. H. Friedan, E.J. Martinec, S. H. Shenker. Nucl. Phys. B. 1986. V. 271. P. 93.

[43] P. Di Vecchia, J. L. Petersen, M. Yu, H. B. Zheng. Phys. Lett. B. 1986. V. 174. P. 280.

[44] Y. Kazama, H. Suzuki. Nucl. Phys. B. 1989. V. 321. P. 232.

[45] V. G. Kač. Infinite dimensional Lie algebras. Cambridge: Cambridge University Press, 1990.

Поступила в редакцию 20.III.1997 г.

\section{A.M. Semikhatov}

\section{REPRESENTATIONS OF INFINITE-DIMENSIONAL ALGEBRAS AND CONFORMAL FIELD THEORY: FROM $N=2$ TO $\widehat{s l}(2 \mid 1)$}

We review a number of constructions, realized in bosonic and $N=2$ strings, whith relate the affine Lie algebra $\widehat{s l}(2)$, affine superalgebra $\widehat{s l}(2 \mid 1)$, and the superconformal $N=2$ algebra. 